Evaluation and Use of the Laboratory Methods in Diagnosis and Control of Tuberculosis

PhD Thesis

Csaba Ködmön

Szeged, 2011 


\title{
Evaluation and Use of the Laboratory Methods in Diagnosis and Control of Tuberculosis
}

\author{
PhD Thesis
}

\author{
by Csaba Ködmön
}

From the European Centre for Disease Prevention and Control, Stockholm, Sweden

Supervisor: Prof. Erzsébet Nagy

Szeged, 2011 


\section{Contents}

1 FOREWORD 1

2 SUMMARY 2

3 ABBREVIATIONS 5

$4 \quad$ AIMS OF THE STUDY $\quad 7$

$\begin{array}{llr}5 & \text { INTRODUCTION } & 8\end{array}$

$\begin{array}{lll}\text { 5.1 The Mycobacterium tuberculosis complex and tuberculosis disease } & 8\end{array}$

$\begin{array}{llr}5.2 & \text { The epidemiology of tuberculosis } & 9\end{array}$

$\begin{array}{lll}5.2 .1 & \text { World } & 9\end{array}$

$\begin{array}{lll}\text { 5.2.2 WHO European Region } & 11\end{array}$

5.2.3 European Union and European Economic Area countries 13

$\begin{array}{llr}5.2 .4 & 16\end{array}$

$\begin{array}{llr}5.3 & \text { Tuberculosis control } & 16\end{array}$

$\begin{array}{lll}\text { 5.3.1 Actions at global level } & 16\end{array}$

5.3.2 The Millennium Development Goals and the Stop TB Strategy 17

$\begin{array}{lll}\text { 5.3.3 The current European context } & 17\end{array}$

$\begin{array}{llr}5.4 & \text { Basics of the laboratory diagnostics of tuberculosis } & 19\end{array}$

$\begin{array}{lll}5.4 .1 & \text { Direct microscopy } & 20\end{array}$

$\begin{array}{llr}5.4 .2 & \text { Culture } & 20\end{array}$

$\begin{array}{lll}\text { 5.4.3 Nucleic acid amplification and molecular biological methods } & 21\end{array}$

6 MATERIALS AND METHODS 23

6.1 Evaluation of the automated Bactec MGIT 960 system versus the semi-automated Bactec 460 TB system and solid medium for isolation of Mycobacteria

6.2 Methods for the $r p o \beta$ gene mutation analysis of the rifampin resistant $M$. tuberculosis strains 24 
6.3 Methods for the molecular epidemiology analysis of the resistant $M$. tuberculosis strains in Hungary

6.4 Methods for the identification of the new $M$. tuberculosis clade among homeless people in Budapest

$7 \quad$ RESULTS

7.1 Evaluation of the automated non-radiometric MGIT 960 system versus the semi-automated radiometric Bactec 12B system and solid medium for isolation of Mycobacteria

7.2 The rpo $\beta$ gene mutation analysis of the rifampin resistant $M$. tuberculosis strains by PCR-based DNA sequencing and reverse-hybridisation

7.3 Molecular epidemiology of resistant M. tuberculosis strains in Hungary

7.4 Identification of a new $M$. tuberculosis strain among homeless people in Budapest

8 DISCUSSION

9 CONCLUDING REMARKS

10 ACKNOWLEDGEMENTS

11 REFERENCES 


\section{Foreword}

This $\mathrm{PhD}$ thesis is based on the following original studies:

I. Somoskövi Ákos, Csaba Ködmön, Ákos Lantos, Zoltán Bártfay, Lilla Tamási, Judith Füzy, Magyar Pál: Comparison of recoveries of Mycobacterium tuberculosis using the Automated Bactec MGIT 960 System, the Bactec 460 TB System, and LöwensteinJensen medium. Journal of Clinical Microbiology, June 2000, 38:2395-2397.

II. Bártfay Zoltán, Ákos Somoskövi, Csaba Ködmön, Nora Szabó, Erzsébet Puskás, Lászlóné Kosztolányi, Eszter Faragó, Judit Mester, Linda M. Parsons and Max Salfinger: Molecular characterization of rifampin-resistant isolates of Mycobacterium tuberculosis from Hungary by DNA Sequencing and the Line Probe Assay. Journal of Clinical Microbiology, 2001, 39:3736-3739.

III. Ködmön Csaba, Stefan Niemann, Judit Lukács, Eva Beer, David Sándor and Ákos Somoskövi: Molecular epidemiology of drug-resistant tuberculosis in Hungary. Journal of Clinical Microbiology, 2006, 44:4258-4261.

IV. Ködmön Csaba, Stefan Niemann, M. Cristina Gutierrez, Christophe Sola, Nalin Rastogi, Judith Lukcs, Ákos Somoskövi: Molecular clues of a homeless microepidemy among tuberculosis patients in Budapest due to a new and local Mycobacterium tuberculosis clade. Infect Genet Evol. 2007, 7:632-5. 


\section{Summary}

Tuberculosis (TB) is among the leading causes of death due to a single pathogen worldwide. The World Health Organization (WHO) estimates that 32\% of the world population is infected with Mycobacterium tuberculosis, the causative agent of tuberculosis, with 9.2 million new TB cases and 1.7 million deaths from TB reported in 2007.

The definitive diagnosis of tuberculosis is dependent on the isolation, identification and drug susceptibility testing of the causal agent Mycobacterium tuberculosis by cultivation. The late 1990's have brought significant changes to clinical mycobacteriology with the introduction of broth-based cultivation and molecular biological methods. However, the value of any new method can be estimated only on the basis of comparisons with conventional techniques currently available in clinical laboratories.

The conventional solid media for culture (e.g. Löwenstein-Jensen medium) is very time-consuming (4-8 weeks) and low sensitivity. Shortening the cultivation time and improvements in the sensitivity of methods are the fundamental needs for laboratory diagnosis of mycobacteria. The liquid-based cultivation systems can satisfactorily fulfil both requirements. The non-radiometric automated Mycobacteria Growth Indicator Tube for (MGIT) (Becton-Dickinson Diagnostic Instrument Systems, Sparks, Md., USA) and the radiometric semi-automatic Bactec 12B (Becton-Dickinson Diagnostic Instrument Systems, Sparks, Md., USA) liquid-based cultivation systems were compared to the conventional Löwenstein-Jensen (LJ) culture method. Both liquid cultivation systems were tested on clinical samples in local conditions for time required and sensitivity for the isolation of mycobacteria. We found that the MGIT and the Bactec 12B culture systems can be easily used, and both are fast and have high sensitivity. Both, the MGIT and the Bactec 12B mycobacterial culture systems are suitable methods for routine laboratory diagnosis of mycobacteria. For the laboratories with low sample-load the use of semi-automatic Bactec 12B system is recommended, while for the high sample-load laboratories the automated MGIT system is more suitable. Furthermore, high sensitivity can be achieved if the liquid-based cultivation system is used in combination with the conventional LJ method.

The recent worldwide increase in the incidence of drug-resistant (DR) strains of Mycobacterium tuberculosis has highlighted the need for faster and more accurate detection of resistance to rifampin (RMP), one of the most important anti-tuberculosis drugs. The 
monoresistance to isoniazid (INH) is quite common, while the RMP monoresistance is very rare. The RMP resistance occurs most often in strains that are also resistant to INH, so RMP resistance is an excellent indicator of the multidrug-resistant tuberculosis (MDR-TB) cases as well.

Therefore, the aim of the present study was to determine the drug resistance profile of 29 RMP-resistant $M$. tuberculosis isolates obtained in East-Hungary and to detect and identify mutations present in the $r p o \beta$ gene. Two molecular assays were used. In the first, two regions of $r p o \beta$ gene that have been associated with RMP resistance were amplified by PCR and the DNA sequence was determined. The results of the DNA sequencing were then compared with results from a commercially available rapid test, the PCR-based reverse hybridization line probe assay (Inno-LiPA Rif. TB Test; Innogenetics N.V., Ghent, Belgium).

In contrast with previous reports, the frequency of occurrence of particular mutations was different in the isolates from East-Hungary, with 11 (37.9\%) isolates carrying the less common D516V mutation. Nine (31.0\%) isolates had an S531L mutation, and two (6.9\%) isolates had an H526D mutation.

The Inno-LiPA Rif. TB Test (LiPA) has been reported to be an easy-to-use test for the rapid detection of RMP resistance. The test is available in a kit format and, therefore, especially useful for routine work in clinical laboratories that are not capable of carrying out DNA sequencing. In the present study, LiPA was able to detect a genetic alteration in 26 (89.7\%) of the 29 RMP resistant strains and to identify the particular mutation in 22 strains (75.9\%). According to the experiences, the LiPA test satisfies the requirements for introduction to the routine laboratory diagnosis in Hungary.

One of the greatest concerns of tuberculosis control programs is the emergence and spread of DR-TB and MDR-TB (resistance at least to isoniazid and rifampin) tuberculosis. In Hungary in 2003, there were 93 (7.9\%) patients with DR-TB (including MDR-TB) and 16 (1.4\%) patients with MDR-TB, and in 2004, the number of DR-TB and MDR-TB cases was 61 (5.1\%) and 9 (0.75\%), respectively. Consequently, the actual extent and type of DR-TB in Hungary are still unknown.

Therefore, a retrospective population-based study was performed to provide a molecular insight into the extent of DR-TB in Hungary using DNA fingerprinting analysis. For this purpose, all drug-resistant Mycobacterium tuberculosis complex strains (68 isolates) 
that were identified in the Hungarian Reference Laboratory for Mycobacteria at the Korányi National Institute for Tuberculosis and Respiratory Medicine in 2003 and 2004 were analyzed by IS6110 restriction fragment length polymorphism (RFLP) fingerprinting and spoligotyping method.

Thirty-one isolates (45.6\%) showed unique fingerprint patterns, while 37 isolates (54.4\%) had an IS6110 RFLP pattern and a spoligotyping pattern identical to those of at least one other isolate and were thus grouped into 12 different clusters. Analysis of the spoligotyping patterns revealed that a remarkably high number (45 [66.2\%]) of patients were infected with strains of the Haarlem genotype, while two (2.9\%) patients were infected with an East African-Indian genotype strain, and no classification of major genotypes was possible for 21 (30.9\%) isolates. It is noteworthy that none of the patients were infected by strains of the Beijing genotype. Statistical analysis did not find any significant difference between clustered and non-clustered patients with regard to their demographic characteristics or their medical and social risk factors. In conclusion, the results of this study indicate that more effective control steps are needed to detect and intercept the transmission of DR-TB in Hungary.

In Budapest, the capital of Hungary, one of the most important tuberculosis related risk factor is homelessness. The aim of this retrospective study was the genetic characterization of Mycobacterium tuberculosis strains isolated from 66 homeless tuberculosis patients by spoligotyping and mycobacterial interspersed repetitive unit (MIRU) typing. The study identified a local microepidemy in the district with the highest tuberculosis incidence in Budapest. Further genetic characterization has shown that the microepidemy was due to a locally emerged Budapest-specific lineage of $M$. tuberculosis. These data may serve as a reference to better monitor and understand the patterns and transmission dynamics of tuberculosis in this at-risk population and is the first report on genetic diversity of M. tuberculosis in today's Hungarians. The findings also indicate that tuberculosis control and prevention steps among the homeless need to be strengthened. 


\section{Abbreviations}

AIDS - Acquired Immunodeficiency Syndrome

AFB - acid-fast bacteria

ANOVA - analysis of variance

BAL - bronchoalveolar lavage

BCG - Bacillus Calmette-Guerin

bp - base pairs

CDC - Centre for Disease Control and Prevention

${ }^{14} \mathrm{CO}_{2}$ - 14-labeled carbon dioxide

CRISPR - Clustered regularly interspaced short palindromic repeats

DNS - deoxyribonucleic acid

DOTS - directly observed therapy short-course

DR - drug-resistant

DRR - direct repeat region

DST - drug susceptibility testing

EAI - East-African-Indian

ECDC - European Centre for Disease Prevention and Control

EEA - European Economic Area

EMB - ethambutol

EU - European Union

GI - Growth Index

HIV - Human Immunodeficiency Virus

HPC - high priority country

INH - isoniazid

LiPA - Inno-LiPA Rif. TB Test

LJ - Löwenstein-Jensen

MDGs - Millennium Development Goals

MDR - multidrug-resistant

MDR-TB - multidrug-resistant tuberculosis

MGIT - Mycobacteria Growth Indicator Tube

MIRU - mycobacterial interspred repetitive unit 
MTBC - Mycobacterium tuberculosis complex

NALC - N-acetyl-L-cysteine

NAT - nucleic acid amplification techniques

$\mathrm{NaOH}$ - sodium hydroxide

NTM - non-tuberculosis mycobacteria

NTSC - National Tuberculosis Surveillance Centre

PCR - polymerase chain reaction

PZA - pirazinamid

RFLP - restriction fragment length polymorphism

RMP - rifampin

SM - streptomycin

SPOLIGOTYPING - spacer oligonucleotide typing

SSA - Sub-Saharan Africa

TB - tuberculosis

UPGMA - Unweighted Pair Group Method with Arithmetic Mean

UV - ultra-violet light

VNTR - variable number of tandem repeat

WHO - World Health Organization

ZN - Ziehl-Neelsen

XDR - extremely drug-resistant

$\chi^{2}$ - Chi square test 


\section{Aims of the study}

I The introduction of new broth-based culture systems for the primary isolation of $M$. tuberculosis at the Laboratory for Mycobacteria of the Department of Respiratory Medicine at Semmelweis University of Medicine:

I/1 Evaluation of the automated non-radiometric Bactec MGIT 960 cultivation system compared with the semi-automatic radiometric Bactec 12B cultivation system and the conventional egg-based Lowenstein-Jensen slant.

I/2 Explore the best available solution for the culturing of Mycobacteria in low- and high sample load laboratories in Hungary.

II Introduction and evaluation of the Inno-LiPA Rif. TB Test [LiPA] (Innogenetics N.V., Ghent, Belgium) assay at the Laboratory for Mycobacteria of the Department of Respiratory Medicine at Semmelweis University of Medicine for the routine direct detection of the mutations in the $r р о \beta$ gene responsible for the rifampin-resistance in $M$. tuberculosis.

III Use of the molecular typing methods in the epidemiology of tuberculosis:

III/1 Introduction and evaluation of the IS6110 RFLP - fingerprint, spoligotyping and MIRU typing methods in Hungary.

III/2 Perform the molecular analysis and describe the molecular epidemic situation of the MDR-TB strains in Hungary. 


\section{Introduction}

\subsection{The Mycobacterium tuberculosis complex and tuberculosis disease}

Members of the Mycobacterium genus widely occur in natural ecosystems. Most members of mycobacteria are decomposing organic matter and due to the nitrogen binding activity they are useful and essential inhabitants of the soil and surface waters. Some Mycobacterium species in the course of evolution has become pathogenic. Among the pathogenic ones the most important are the members of Mycobacterium tuberculosis complex (MTBC), especially $M$. tuberculosis and $M$. bovis. There are significant differences in the virulence and the host specificity among the complex members (M. tuberculosis, M. bovis, M. bovis bacillus Calmette-Guerin BCG, M. africanum, M. microti, M. canettii, M. bovis subsp. caprae) [1]. M. tuberculosis, M. africanum and $M$. canettii are primarily human pathogens [2], M. microti mainly causes disease in rodents, although a few human cases have been reported as well. $M$. bovis can cause disease in both humans and animals (cattle, goats, elephants, deer, cats, seals, etc.) [3], however the infection is more frequent in animals. Bacillus Calmette-Guerin is an attenuated vaccine strain, which can cause disease by shocking of the immune system [4]. M. tuberculosis and $M$. bovis are the best examples of the differences in host specificity. The number of human infections caused by M. bovis is much lower compared to the number caused by M. tuberculosis. In addition, it was observed that transmission of the disease among close contacts is significantly lower in case of the M. bovis infection than in case of $M$. tuberculosis [5]. Although the phenotypic similarity is less than $65 \%$ among the members of the complex, there is about $99.9 \%$ genetic identity observed at deoxyribonucleic acid (DNA) level [6]. Thus, these organisms are more subspecies rather than individual species.

The disease caused by infection with Mycobacterium tuberculosis complex members is called tuberculosis. The most common form is pulmonary tuberculosis where at the site of infection the production of various mediators, the activation and accumulation of alveolar macrophages and lymphocytes can be observed, leading consequently to a helper $\mathrm{T}$ lymphocyte/macrophage alveolitis $[7,8]$. The course and progression of disease is depending on interaction the pathogen of the various regulatory mechanisms for induction of cytokine production of the host. The cytokine release rate at the site of infection in the lungs, the release rate of $\mathrm{T} 1$ helper and $\mathrm{T} 2$ helper type cytokines in broncho-alveolar lavage (BAL) cells 
(alveolar macrophages and lymphocytes) and in circulating mononuclear cells correlates with the severity of the disease [7, 9-11]. In contrast, the secondary immune response function and the expression of molecules (CD58, CD80, CD86 and HLA-DR) in signal transduction system has shown that the activity of alveolar macrophages is not dependent on severity of disease [10]. This indicates that the aggravation is not a consequence of an immune-paralysis.

As the symptoms of tuberculosis are not specific, laboratories have a vital role in the diagnosis of tuberculosis, in the monitoring and determining of the effectiveness of therapy used as well as in preventing the transmission of disease. The chest X-ray examination provides a good basis for diagnosis of suspected tuberculosis; however lot of lung diseases may show a similar picture on the radiograph. Therefore, the laboratory findings must be accurate and also readily available.

\subsection{The epidemiology of tuberculosis}

\subsubsection{World}

The World Health Organization declared a global emergency on tuberculosis in 1993. In 2008, there were an estimated 8.9-9.9 million incident cases of TB, 9.6-13.3 million prevalent cases of TB, 1.1-1.7 million deaths from TB among HIV-negative people and an additional 0.45-0.62 million TB deaths among HIV-positive people (classified as HIV deaths in the International Statistical Classification of Diseases), with best estimates of 9.4 million, 11.1 million, 1.3 million and 0.52 million, respectively [12]. The number of notified TB cases in 2008 was 5.7 million, equivalent to $55-67 \%$ of all incident cases, with a best estimate of $61 \%$ (10\% less than the Global Plan milestone of a case detection rate of $71 \%$ in 2008). Among patients in the 2007 cohort, 87\% were successfully treated; this is the first time that the target of 85\% (first set in 1991) has been exceeded at global level. Progress in implementation of interventions to reduce the burden of TB in HIV-positive people has continued; in 2008, 22\% of TB patients knew their HIV status (up from 20\% in 2007) including $45 \%$ of patients in the African Region; 0.3 million people were enrolled on cotrimoxazole preventive therapy; and 0.1 million people were enrolled on antiretroviral therapy. Almost 30.000 cases of multidrug-resistant TB (MDR-TB) were notified in 2008; this is $11 \%$ of the total number of cases of MDR-TB estimated to exist among cases notified in 2008. Most of the estimated number of cases in 2008 occurred in Asia (55\%) and Africa 
(30\%), with small proportions of cases in the Eastern Mediterranean Region (7\%), the European Region (5\%) and the Region of the Americas (3\%). The 22 high-burden countries (HBCs, defined as the countries that rank first to $22^{\text {nd }}$ in terms of absolute numbers of cases and which have received particular attention at the global level since 2000) account for $80 \%$ of all estimated cases worldwide (Map 1). The five countries that rank first to fifth in terms of total numbers of incident cases in 2008 are India (1.6-2.4 million), China (1.0-1.6 million), South Africa (0.38-0.57 million), Nigeria (0.37-0.55 million) and Indonesia (0.34-0.52 million). India and China alone account for an estimated 35\% of TB cases worldwide.

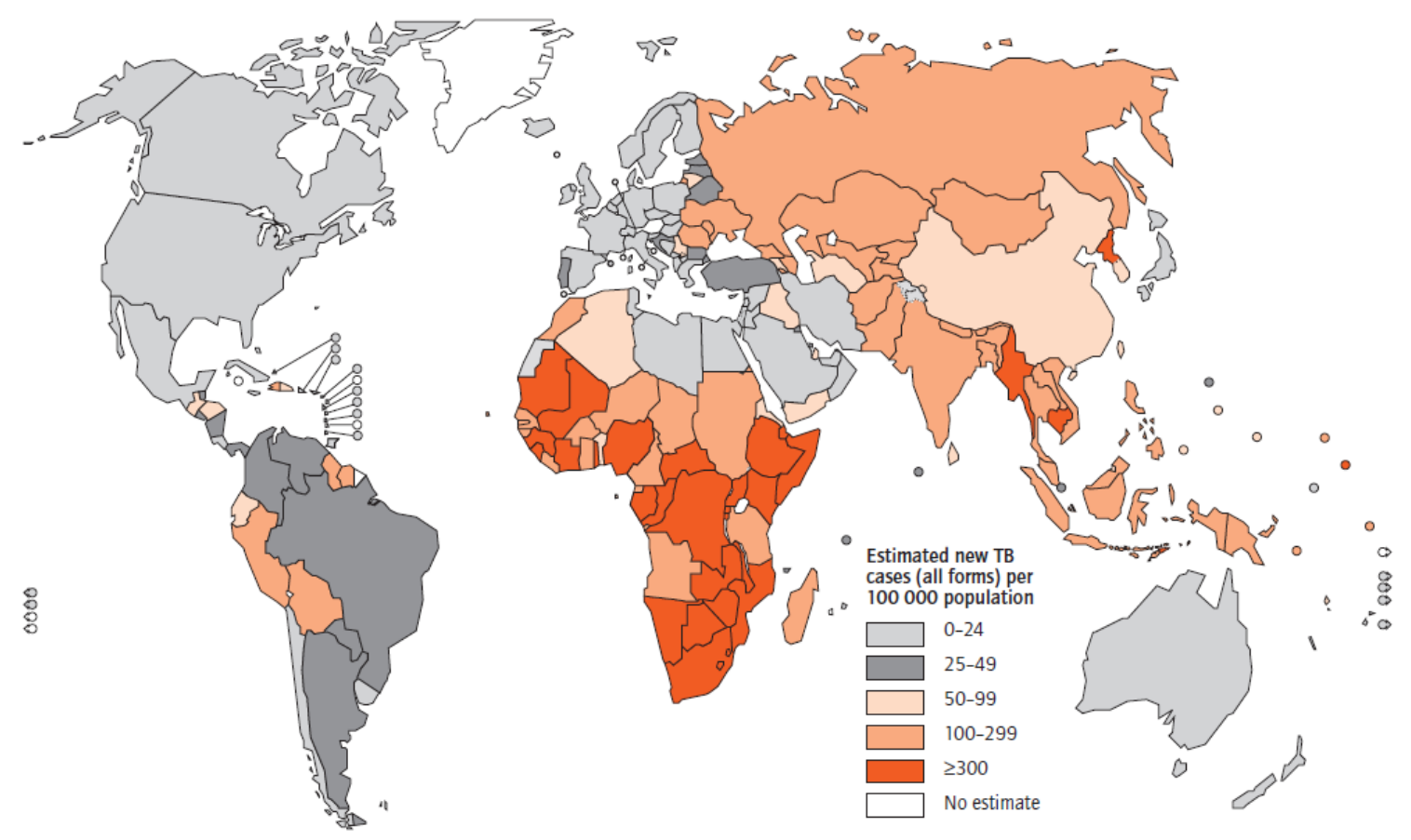

Map 1. Estimated TB incidence rates, 2008. [12]

The main driving forces to the global TB epidemic are the socio-economical conditions, the increasing HIV burden and the increasing resistance, especially MDR-TB among TB strains. The lack of effective national tuberculosis programs, the poor implementation of the control measures, the low quality and accessibility of anti-TB drugs, the use of not appropriate treatment regiments and the poor patient compliance lead to increased emergence of MDR-TB as well as the extremely drug-resistant (XDR) TB in the world $[13,14]$. A recent mathematical modelling shows that the successful prevention of the 
outbreaks and epidemics of MDR-TB requires detection at least $70 \%$ of infectious (microscopically positive) cases of MDR-TB each year with proper bacteriological identification and drug resistance results as well as successful treatment of $80 \%$ of the cases [15]. However, there are a number of difficulties in the treatment of MDR-TB cases. The so-called first-line anti-TB drugs (INH, streptomycin [SM], RMP, pirazinamid [PZA] and ethambutol [EMB]) are no longer suitable treatment of the MDR-TB patients, and therefore it requires the use of second-line anti-TB drugs [16]. In many parts of the world is difficult to obtain the second-line anti-TB drugs, and often those are not of sufficient quality. Furthermore, the second-line anti-TB therapy has significantly higher costs and the sued agents are more toxic as the first-line anti-TB drugs [17]. It should be mentioned that the drug susceptibility testing (DST) for the second-line anti-TB drugs are performed in sub-optimal number of places. Due to all those factors the patients infected with the resistant M. tuberculosis are remaining infectious significantly longer than those infected with the sensitive strain.

\subsubsection{WHO European Region}

In 2008, 461.645 TB cases (52,2 per 100.000 population) were reported by 50 of the 54 countries of the European Region1, representing approximately 6\% of TB cases reported worldwide to WHO [12] (Map 2.).

\footnotetext{
1 No data from Monaco, San Marino, Austria or Liechtenstein; Liechtenstein is included in this report, but is only presented as and EEA country as it is not a WHO European Region Member State.
} 


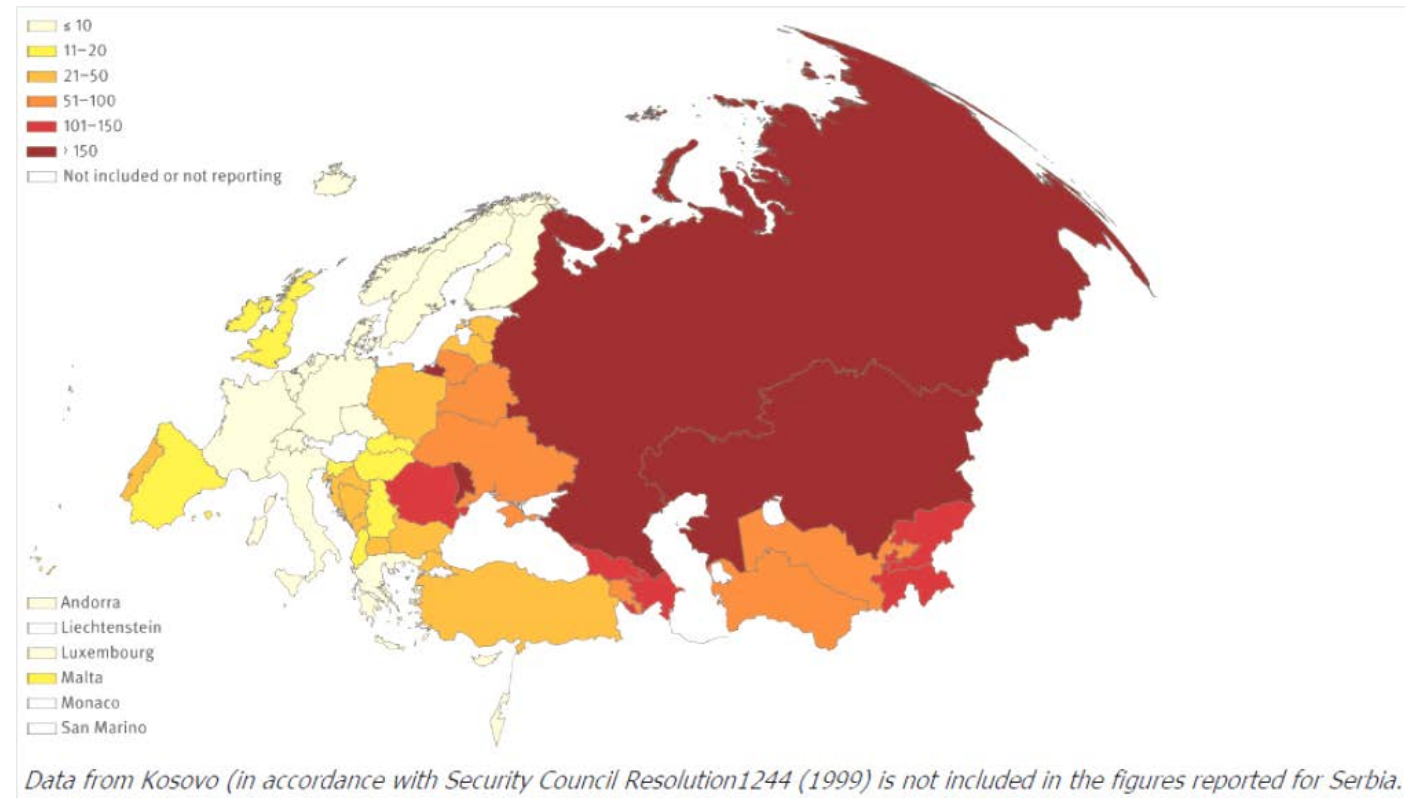

Map 2. Total TB notification rates per 100000 population, Europe, 2008 [18]

The trend in overall TB notification rates in the European Region has continued to increase since 2004. However, overall TB notification rates across the 18 high priority countries (HPC) ${ }^{2}$ decreased from the previous year by $3.9 \%$ to $87.6 \%$ of all TB cases and across the Region the overall notification decreased by 2,6\% between 2007 and 2008. A decrease by 4\% from 2007 in the notification of newly detected TB cases suggests reduced spread of TB in the Region. The percentage of previously treated cases has also decreased since 2007 from $31.7 \%$ to $29.8 \%$. The age group with the highest number (42.0\%) of newly detected TB cases in the Region is 25-44 years. The number of reported HIV co-infected TB cases almost doubled, from 5.828 in 2006 to 11.395 in 2008, due to increased testing as part of intensified HIV care services for TB patients in the HPCs. There was no appreciable increase in the number of reported HIV co-infections outside of the HPCs during this period. Across the European Region, the total number of reported MDR-TB cases for 2008 has doubled since the previous year due to improvements in DST. The MDR-TB prevalence among new TB cases was at $11.1 \%$. The highest burden of MDR-TB cases in the European Region is found in the HPCs, with a prevalence of $13.8 \%$ among newly diagnosed cases, 2 Armenia, Azerbaijan, Belarus, Bulgaria, Estonia, Georgia, Kazakhstan, Kyrgyzstan, Latvia, Lithuania, Moldova, Romania, Russia, Tajikistan, Turkey, Turkmenistan,
Ukraine, Uzbekistan. 
which is five times higher than the prevalence reported in the EU/EEA ${ }^{3}$, and over $50 \%$ among previously treated cases, which proportion is more than twice as high as in the EU/EEA.

The treatment success rate among the newly detected laboratory-confirmed TB cases in 2007 has decreased to the level of 70.7\% (compared with 73.1\% for cases registered in 2006); 9.0\% were reported as failed treatment, $8.4 \%$ died, and $6.9 \%$ defaulted. The treatment success rate in non-EU/EEA countries is lower than in the EU/EEA: 67.5\% compared with $79.5 \%$, respectively. In the 18 HPCs, treatment succeeded for only $69,2 \%$ of newly detected laboratory confirmed TB cases, which is far from the 85\% Stop TB Strategy target [19]. The TB mortality rate has decreased by $45 \%$ from 9.0 per 100.000 of population in 2005 to 5.0 per 100.000 of population in 2007. Similar proportional reductions were seen in the EU/EEA as well as the HPCs. However, mortality in the HPCs remained almost 15 times higher than in the EU/EEA.

\subsubsection{European Union and European Economic Area countries ${ }^{4}$}

For 2008, 82.611 TB cases were reported by 26 European Union (EU) countries (all except Austria) and two other countries of the European Economic Area (EEA) (Iceland and Norway), showing a decrease of 615 cases compared with 2007 (Figure 1).

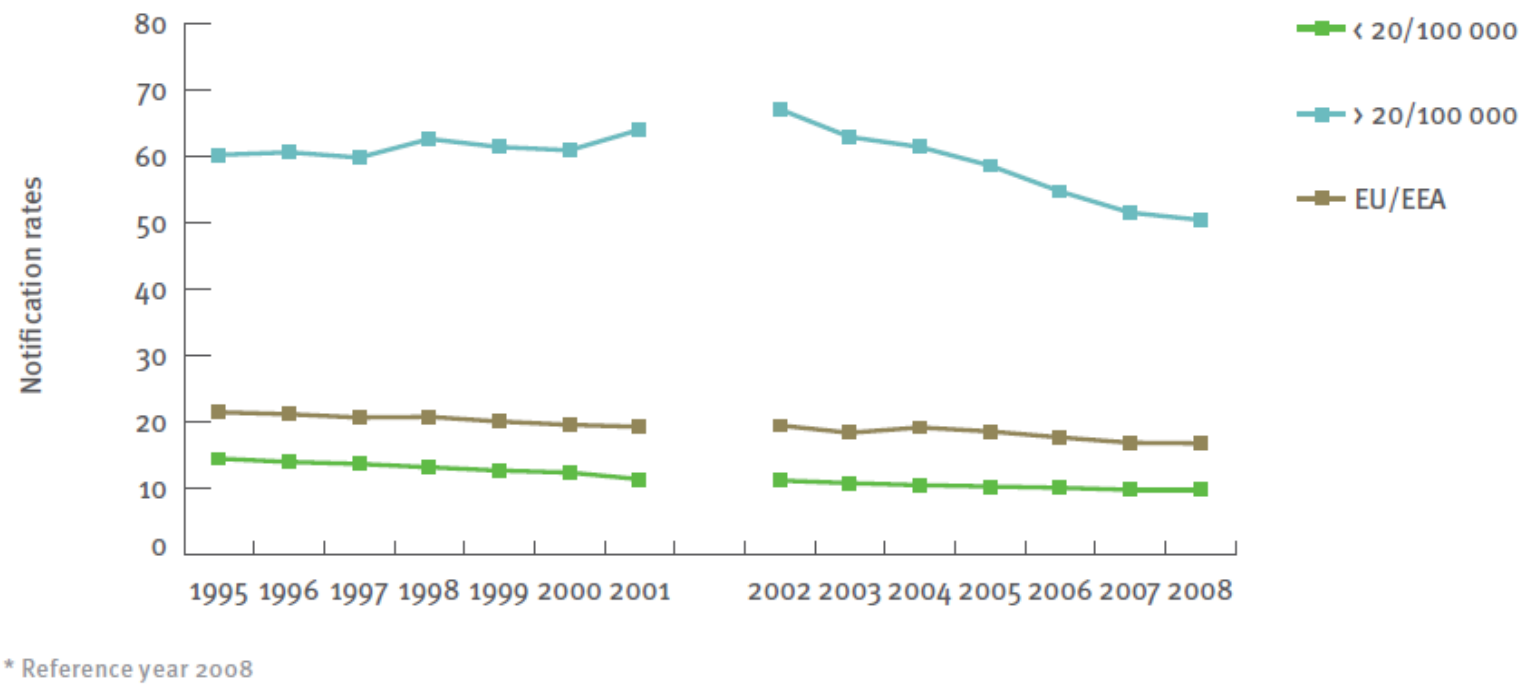

Figure 1. TB notification rates by incidence grouping*, 1995-2001 and 2002-2008 [18]

\footnotetext{
3 The 27 EU Member States, Iceland, Liechtenstein and Norway.

4 The 27 EU Member States, Iceland, Liechtenstein and Norway.
} 
Over $80 \%$ of cases occurred in the eight countries that reported 3.000 cases or more each (Bulgaria, France, Germany, Italy, Poland, Romania, Spain and United Kingdom) (Figure 2).

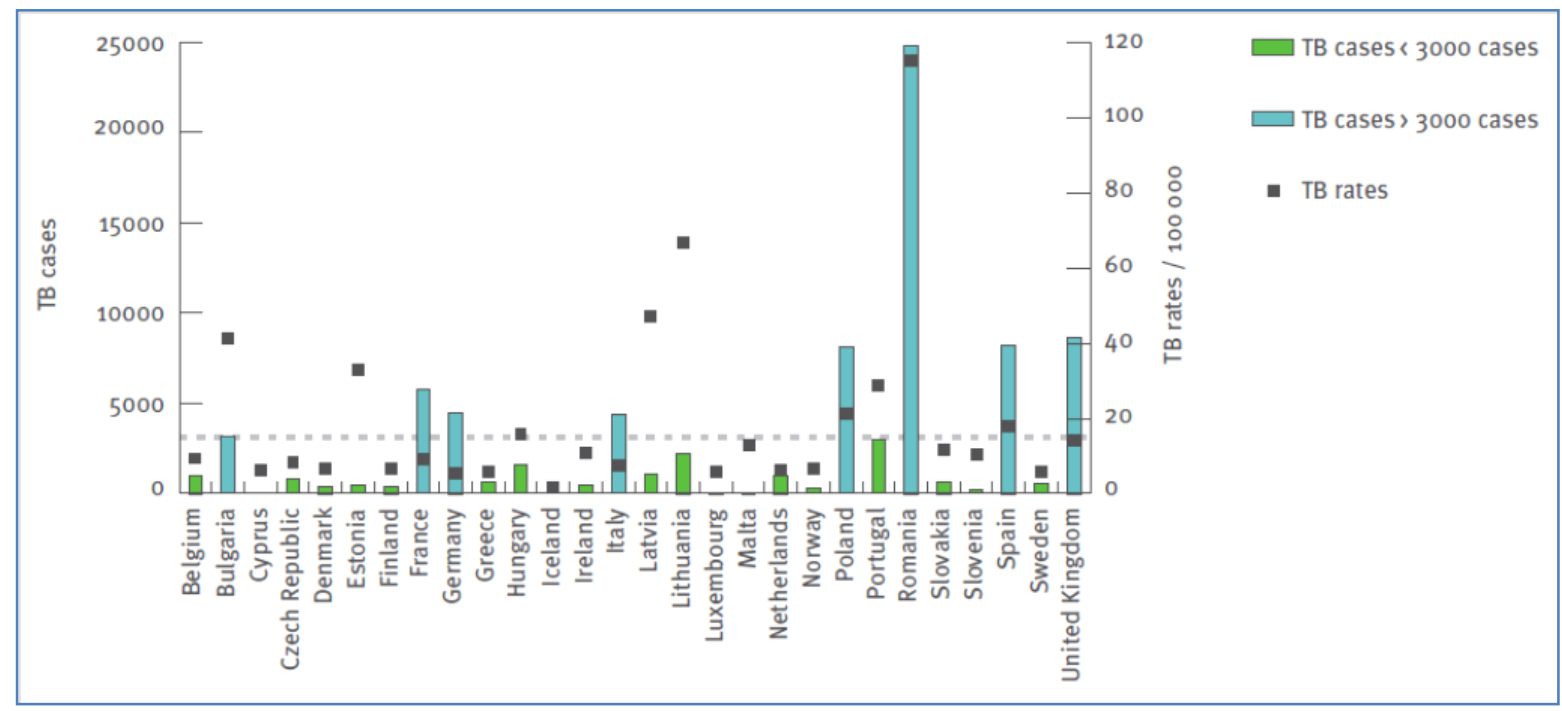

Figure 2. EU/EEA caseload burden 2008 by Member State [18]

The overall notification rate in 2008 was 16.7 per 100.000, with rates lower than 20 per 100.000 reported in 21 countries and higher than 20 per 100.000 in Romania $(115,1)$, the Baltic States - Lithuania (66.8), Latvia (47.1), Estonia (33.1) - Bulgaria (41.2), Portugal (28.2) and Poland (21.2). The overall notification rate was 1.2\% lower than that in 2007 (for the 28 reporting countries), reflecting a net downward trend in 17 countries. However, substantial increases were observed in Malta (28.8\%), Iceland (19.8\%) and Cyprus (12.2\%), and some increases were seen in Sweden (4.9\%) and in the United Kingdom (2.8\%), mostly of foreign origin cases. In 2008, 22.4\% of cases (country range: 0-88\%) were in persons of foreign origin (Figure 3), more than two-thirds of whom originated from Asia or Africa. 


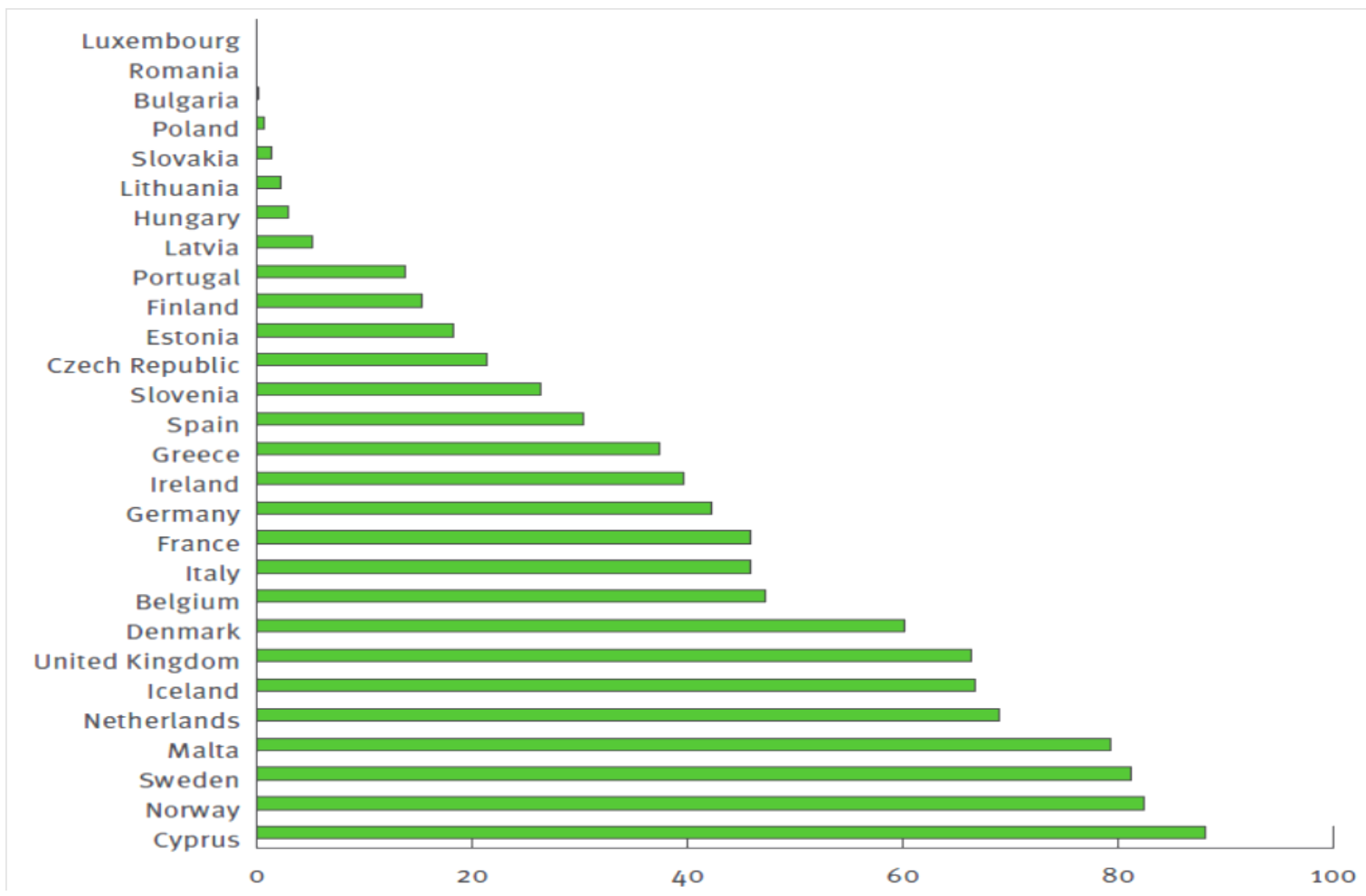

Figure 3. Proportion of foreign-origin cases as percentage of all TB cases, 2008 [18]

HIV prevalence among TB cases increased between 2006 and 2008 in Estonia (from 9,0\% to 9.9\%), Latvia (3.4\% to 6.7\%) and Malta (3.7\% to 9.4\%). In the rest of the countries that submitted data, the HIV prevalence among TB cases was $1 \%$ or less in six countries, $2-5 \%$ in three others, $5-8 \%$ in two countries and $14.6 \%$ in one country. Multidrug resistance remained most frequent in the Baltic States (combined MDR: 15.6\%-21.3\%) followed by Romania, which reported results for the first time (14.7\%). Other countries reported lower levels of MDR (0\%-5\%), where it was generally more common in cases of foreign origin. Of the 14 countries reporting XDR, Romania had the highest numbers (total of 54 cases), while Latvia and Estonia had the highest percentage of XDR cases among MDR cases (14.7 and $12.2 \%$, respectively) with Latvia showing a sharp increase in XDR cases compared with 2007, from 6 to 19 (6.1\% to 14.7\%). Twenty-two countries reported treatment outcome monitoring data for definite pulmonary TB cases in 2007. Among previously untreated, culture-confirmed, pulmonary TB cases, $79.5 \%$ had a successful outcome. Successful outcomes were significantly lower among previously treated TB cases $(51,8 \%)$ and among MDR-TB culture-confirmed pulmonary cases at 24 months (30.9\%) [18]. 


\subsubsection{Hungary}

In Hungary, after 20 years of decline, between 1990 and 1995 the incidence of tuberculosis displayed an increase of almost 25\% (rising from 31/100.000 to 39/100.000) [20]. To stop the negative trend, in 1994 the National Tuberculosis Program was developed to optimised the efforts in fight against TB in Hungary. Since 1996 the incidence rate is steadily decreasing and in 2009 reached the level of 16.0/100.000 of population. Despite the significant in incidence the number of fatal TB cases remains almost the same. This phenomenon suggests an aggravation of disease among newly diagnosed patients due to the low cooperation level of the patients and the not appropriate case management. One of the main gaps in TB control is the low proportion of the bacteriologically confirmed cases. According to the data reported to supranational level it was never over $50 \%$ at all. However, the anti-TB drug resistance in low in Hungary where 16 (2.7\%) MDR-TB cases were reported in 2008. The treatment success rate of all pulmonary bacteriological conformed cases was $48.5 \%$ which remains still suboptimal compared to the global target of $85 \%$.

The homelessness is one of the major risk factors in the big cities and industrial centres. Budapest, as the largest city and economical centre has the largest number of homeless population in Hungary. In 2002, the incidence of tuberculosis in the homeless population was 676/100.000, and reached to $726 / 100.000$ in 2004 [21, 22]. In 2004, the 53\% of TB cases in homeless population was reported from Budapest, which represents the $22.3 \%$ of the total TB cases in the city [21].

\subsection{Tuberculosis control}

\subsubsection{Actions at global level}

Tuberculosis was explicitly recognised as a major global public health problem in the early 1990s, when the World Health Assembly adopted a resolution calling for increased efforts to control the disease. The resolution set two major targets for global action against TB: the detection of $70 \%$ of new smear-positive cases, and cure of $85 \%$ of such cases. In 1994, the internationally recommended control strategy, directly observed therapy shortcourse (DOTS), was launched, followed in 1998 by the Stop TB Initiative. In 2000, this became the Stop TB Partnership, a global network of international organisations, countries, sponsors, non-governmental organisations and other interested parties committed to 
controlling and eventually eliminating TB. The first Stop TB Partners' Forum, held in Washington in 2001, launched a Global Plan to Stop TB for the period 2001-05. Subsequently, a plan for 2006-15 was launched in January 2006, and more recently an updated version for the period 2011-15 has been developed. These global plans form the overarching framework for the Stop TB Partnership’s combined actions.

\subsubsection{The Millennium Development Goals and the Stop TB Strategy}

The adoption of the Millennium Development Goals (MDGs) by the global community in 2000 provided renewed impetus for TB control efforts. It calls for action to combat HIV/AIDS, malaria and other diseases, including TB. Widespread implementation of the DOTS strategy led to significant progress in control of the disease. For example, by 2007 the treatment success rate among new smear-positive cases had reached $86 \%$, while in 2008, the global case detection rate was 61 \% [23]. However, by 2005, it had become clear that DOTS alone would not be sufficient to achieve global TB elimination. In 2006, therefore, the Stop TB Strategy was launched, designed to meet both the TB-related MDGs targets and the Stop TB Partnership targets for 2015. The Stop TB Strategy underpins the Global Plan to Stop TB 2006-2015 [24].

\subsubsection{The current European context}

The goals and targets described in the MDGs and the Stop TB Strategy have been endorsed by European countries. However, because the MDGs in particular were conceived in a context of promoting development and eradicating poverty, these targets are not necessarily optimally adapted to the European context. For example, many European countries are already seeing a steady decline in TB case notification rates, and have in place the infrastructure and personnel needed to implement the necessary TB control activities. The impact of the global targets in promoting and motivating TB control in Europe has therefore been limited. Nevertheless, it has to be recognised that, with current trends, Europe is unlikely to achieve elimination of the disease by the target date of 2050. Imported cases and drug resistance will continue to pose challenges, even for low-incidence countries, and will require concerted action on a broad front, tailored to the specific situation. Recognition of the particular challenges to TB control in Europe has led recently to a number of broad-based initiatives, as outlined below. 


\section{The Berlin Declaration}

In October 2007 in Berlin, Germany, the WHO Regional Office for Europe convened a Ministerial Forum on Tuberculosis, to seek increased commitment from governments to work together to tackle the TB epidemic in the Region. In the resulting Berlin Declaration [25], countries undertook, inter alia, to strengthen their public health and social services systems to respond to the TB situation in the Region, to adopt the Stop TB Strategy, and to seek sustainable funding for TB control efforts. The Declaration effectively reset TB control agendas across the European Region.

\section{The plan for high-priority countries in Europe}

Recognising that, within the European Region the countries of eastern Europe were hardest hit by TB, the WHO Regional Office in 2007 produced a plan to tackle the disease in those countries [26]. Of the 18 countries targeted in the plan, five - Bulgaria, Estonia, Latvia, Lithuania, and Romania - are members of the EU. The purpose of the plan was to contribute to the achievement of six specific targets for 2010:

I. To reach $100 \%$ DOTS population coverage in all eastern European countries.

II. To increase the case detection rate of new infectious (sputum smear-positive) TB cases to at least $73 \%$.

III. To achieve treatment success in at least $85 \%$ of detected new infectious TB cases.

IV. To provide treatment according to internationally recommended guidelines for $100 \%$ of multidrug-resistant TB cases (new and previously treated).

V. To reduce the prevalence of TB (all forms) to 188 cases per 100000 of population.

VI. To decrease the mortality rate from TB (all forms) to 16 deaths per 100000 of population.

\section{The Framework Action Plan to Fight TB in the EU}

Also in 2007, European Centre for Disease Prevention and Control (ECDC) began to develop a framework for action to be taken by and within countries, as well as at European Community level, to combat TB [27]. The Action Plan, which is compatible with both the Global Plan to Stop TB and the plan for high-priority countries in Europe, is based on four principles: (i) ensuring prompt and quality care for all; (ii) strengthening capacity of health systems; (iii) developing new tools; and (iv) building partnerships and collaboration with countries and stakeholders. Eight areas for strategic development are organised around these 
four principles, and recognise the need to consider the heterogeneous epidemiological picture in the EU and the different needs of countries with high or low TB incidence.

The areas are:

Area 1. TB control commitment, TB awareness and capacity of health systems

Area 2. Surveillance

Area 3. Laboratory services

Area 4. Prompt and quality TB care for all

Area 5. MDR- and XDR TB

Area 6. TB/HIV co-infection

Area 7. New tools for TB control

Area 8. Build partnership and collaboration with countries

\subsection{Basics of the laboratory diagnostics of tuberculosis}

Laboratory methods play a crucial role in establishing the diagnosis of tuberculosis, monitoring the therapy and preventing transmission of the disease, because the clinical signs and symptoms of the disease are not specific enough. The deviations on chest radiographs may strongly suggest the presence of tuberculosis, but other lung diseases may give rise to similar findings. In addition, chest radiographs cannot distinguish between current active tuberculosis and previously occurred infection. Laboratory results must be not only accurate, but also available in the shortest time possible. However, the laboratory techniques most commonly used worldwide for the diagnosis of tuberculosis were developed in the $19^{\text {th }}$ century and have been modified only slightly. The recent re-emergence of tuberculosis has highlighted the shortcomings of the laboratory diagnosis of tuberculosis. It is generally accepted that the use of a combination of liquid and solid media is the gold standard for the isolation of mycobacteria $[28,29]$. The last few years of $20^{\text {th }}$ century with the introduction of broth-based cultivation and molecular biological methods the significant changes and improvements have been brought to laboratory methods used. However, the value of any new method can be assessed only by comparison with conventional techniques currently available in laboratories. 


\subsubsection{Direct microscopy}

Acid-fast microscopy is still the easiest, cheapest and most rapid procedure in mycobacteriology. It has a specificity of over 99\% (for Mycobacterium sp.), it requires standard reagents with long shelf-lives and it can be applied successfully in any laboratory. However, the sensitivity of microscopy is not satisfactory. When a single smear of a respiratory specimen is examined, acid-fast microscopy provides false-negative results in $25 \%$ to $50 \%$ of patients with active tuberculosis [30,31]. Thus, a positive smear test of a respiratory specimen is useful in making a presumptive diagnosis of tuberculosis, a negative test does not rule out the disease. Microscopic examination does not allow a differentiation between viable and non-viable bacteria, or the identification of different mycobacteria species. The staining of smears with a fluorochrome (e.g. auramine) is superior to the classical Ziehl-Neelsen (ZN) or Kinyoun method, since a lower magnification can be used with fluorescence microscopy, which reduces the time demanded for screening. Unfortunately, the good laboratory practice requires the confirmation of any fluorescence microscopy smear-positive results by a ZN stain [32]. Thus, the initial advantage of the faster detection of acid-fast bacteria (AFB) by fluorescence microscopy is reduced. However, the careful examination of smears is time-consuming and labour-intensive in laboratories with very high daily volumes. It is clear that, while acid-fast microscopy is an important adjunct to the detection of mycobacteria, it is not an adequate criterion alone and must be followed by culture.

\subsubsection{Culture}

The yield of cultivation for the detection of M. tuberculosis is higher than that of acid-fast microscopy. Historically, the egg-based media, such as that of LJ, are the bestknown of the solid media used for the isolation of $M$. tuberculosis. The agar-based media, e.g. Middlebrook 7H10 and 7H11 agar, offer a better opportunity for the examination of colonial morphology and the detection of mixed cultures than do egg-based media [33, 34]. However, on both type of the conventional solid media, colonies of $M$. tuberculosis are rarely visible before 6-8 weeks of incubation [35]. The identification and determination of the drug susceptibility of a mycobacterium isolate by culturing can add 4-6 weeks to this already long process. It can be 2-3 months before the results of identification and drug susceptibility tests 
are available [36]. Moreover, it has been observed that as many as $29.5 \%$ of patients reported to have tuberculosis are not culture-confirmed when solid media are used alone. The use of a liquid medium is generally recommended for subcultures and in vitro tests (i.e. DST). A liquid medium can be used for the primary isolation of $M$. tuberculosis from sputum only if it is a selective medium (containing incorporated antibiotics) [37]. In selective liquid media, the growth of $M$. tuberculosis can be detected within 1 to 2 weeks, depending on the number of bacteria in the specimen. Furthermore, even if no growth is detected in the liquid media, the final report that the specimen is culture-negative can be issued only after 8 weeks of incubation of the LJ slants. Further development and introduction of new cultures and other direct detection methods are therefore necessary for the more rapid, more reliable and less labour-intensive detection of mycobacteria.

\subsubsection{Nucleic acid amplification and molecular biological methods}

The use of nucleic acid amplification (NAT) methods in the routine detection of mycobacteria allowed a fast and accurate detection of the Mycobacterium species within 24 hours [38, 39]. Using the nucleic acid amplification techniques, in theory, a single mycobacterial DNA can be amplified with an enzymatic reaction to the detectable amount in a very short period of time. The nucleic acid amplification techniques and other molecular biology methods (i .e. DNA hybridization, DNA sequencing, etc.) are essential in today’s laboratory practices for detection and characterization of mycobacteria. The methods are widely used for the identification of mycobacteria, detection of the mutations in the resistance genes as well as the molecular epidemiological studies. The molecular epidemiology requires a reproducible and comparable typing method with an optimal discriminatory power.

Spacer oligonucleotide typing (spoligotyping) is a hybridization assay that detects variability in the direct repeat region (DRR) in the DNA of $M$. tuberculosis. The DRR consists of multiple copies of a conserved 36-base-pair sequence (the direct repeats) separated by multiple unique spacer sequences (the standard spoligotyping assay uses 43). Different $M$. tuberculosis strains have various complements of the 43 spacers, and these different complements form the basis of the assay. The spoligotyping involves polymerasechain-reaction (PCR) amplification of the DR locus, followed by hybridization of the labeled PCR products to a membrane that contains covalently-bound oligonucleotides corresponding to each of 43 spacers. Individual strains have positive or negative signals for each spacer. 
However, the spoligotyping has the less discriminatory factor as the Variable number of tandem repeat (VNTR) typing and the IS6110 restriction fragment length polymorphism (RFLP) typing methods; it is widely used as the $2^{\text {nd }}$ typing method.

The VNTR typing is based on analysis of DNA segments containing "tandem repeated" sequences in which the number of copies of the repeated sequence varies among strains. The method relies on PCR amplification and calculation of the number of repeats on the basis of the size of the amplified product. The mycobacterial interspred repetitive unit (MIRU) is a class of tandem repeated sequences. There are a total of 41 MIRU loci, of which 24 have been selected for genotyping.

IS6110 RFLP genotyping detects variations generated by the insertion element IS6110. Insertion elements are capable of making copies of themselves and then inserting the copy anywhere in the genome in a process known as transposition. Strains can differ in both the number of copies of IS6110 and the positions of IS6110 in the bacterial DNA. 


\section{Materials and methods}

\subsection{Evaluation of the automated Bactec MGIT 960 system versus the semi-automated Bactec 460 TB system and solid medium for isolation of Mycobacteria}

A total of 377 consecutive clinical specimens (288 sputum, 51 BAL or bronchial mucus aspirate, 32 gastric juice, and 6 pleural effusions) from 243 patients were processed between 29 March 1999 and 31 May 1999. All patients were human immunodeficiency virus negative. All clinical specimens were digested and decontaminated by the N-acetyl-Lcysteine-NaOH (NALC) method as described by Kent and Kubica [37]. A 4\% concentration (starting concentration) of sodium hydroxide $(\mathrm{NaOH})$ was used giving the $2 \%$ final concentration in the decontamination mixture. After decontamination, smears were prepared from the concentrated sediments of the specimens for $\mathrm{ZN}$ acid-fast staining. The remaining sediment was suspended in $1.5 \mathrm{ml}$ of sterile phosphate-buffered saline ( $\mathrm{pH}$ 6.8). Before inoculation, Bactec MGIT 960 and Bactec 12B (used for Bactec 460 TB system) vials were supplemented as described by the manufacturer. We inoculated $0.5 \mathrm{ml}$ of the processed specimen into the non-radiometric Bactec MGIT 960, $0.5 \mathrm{ml}$ into the radiometric Bactec 12B, and $0.2 \mathrm{ml}$ onto each of two LJ medium slants. All inoculated media were incubated at $37^{\circ} \mathrm{C}$. Bactec MGIT 960 vials were introduced into the Bactec MGIT 960 instrument as recommended by the manufacturer and tested either until they were found to be positive or for 6 weeks. The Bactec 12B vials were read twice weekly for the first 2 weeks and weekly thereafter for 4 weeks. When the growth index of a Bactec $12 \mathrm{~B}$ vial reached $\geq 10$, the vial was tested daily until the vial attained a growth index of $\geq 100$, at which time it was considered presumptively positive. If no 14-labeled carbon dioxide $\left({ }^{14} \mathrm{CO}_{2}\right)$ production was detected after 6 weeks, the Bactec 12B vial was regarded as negative. The LJ medium slants were examined weekly for 8 weeks for the visible appearance of colonies. After confirmation of mycobacterial growth in a liquid or solid medium, the parallel media were read daily. On the day of detection, all positive liquid and solid media were examined by ZN staining to confirm the presence of AFB and sub-cultured onto Columbia agar with 5\% sheep blood (BioMerieux Microbiology Systems, Marcy l'Etoile, France) to check for contaminants. Cultures found AFB positive by microscopy were identified by means of the AccuProbe culture identification test (Gen-Probe, San Diego, Calif.) and conventional biochemical tests [37]. 
The $\chi 2$ test was used to evaluate differences between recovery rates in different media. Analysis of variance (ANOVA) and the Newman-Keuls test were used to establish significant differences in relation to the duration of growth.

\subsection{Methods for the $r p o \beta$ gene mutation analysis of the rifampin resistant M. tuberculosis strains}

The 29 RMP-resistant strains isolated in 1999 were examined. The M. tuberculosis H37Rv ATCC 27294 reference strain and six clinical M. tuberculosis isolates that were pansusceptible for all four first-line anti-tuberculosis drugs were used as controls. All cultures were identified by means of the AccuProbe culture identification test (Gen-Probe Inc., San Diego, Calif.) and conventional biochemical tests [1, 37]. Susceptibility testing of all 36 isolates for INH, RMP, EMB, and SM was carried out by the proportion method on Löwenstein-Jensen medium as described by Canetti et al. [40]. The critical concentrations for INH, RMP, EMB, and SM were 0.2, 40, 1.0, and $10 \mu \mathrm{g} / \mathrm{ml}$, respectively. On the day of detection of growth index 999, 200- $\mu$ l aliquots from Bactec 12B subcultures of the susceptible control and RMP-resistant isolates were incubated at $80^{\circ} \mathrm{C}$ for one hour to heat kill the mycobacterial cells. Using primers rpo95 (5`-CCACCCAGGACGTGGAGGCGATCACACCG-3`) and rpo397 (5`-GTCAACCCGTTCGGGTTCATCGAAACG-3`) flanking the 81-bp region of rро $\beta$ [41], a 329-bp product was generated from all 36 isolates. The same primers were used for DNA sequencing of both strands using the automated Applied Biosystems 377 DNA sequencer (Applied Biosystems, Foster City, Calif.). A recent study demonstrated that, in some RMP-resistant strains with the wild-type sequence in the 81-bp region, a V146F mutation was found in the N-terminal region [42]. In order to detect the presence of this mutation, amplification and sequencing were performed using primers Tb176-f (5`-CTTCTCCGGGTCGATGTCGTTG-3`) and Tb176-r (5`-CGCGCTTGTCGACGTCAAACTC-3`) as described previously [42]. A 365-bp product was generated and sequenced using the same primers. The heat-killed samples were also used for production of a biotinylated 256-bp fragment of the rpo $\beta$ gene using the LiPA kit according to the instructions of the manufacturer (Innogenetics N.V., Ghent, Belgium). The biotin-labelled PCR product was denatured and hybridized to a strip with 10 specific oligonucleotide probes (19 to 23 bases long). One probe is specific for the $M$. tuberculosis complex (TB-P), while 
five partially overlapping wild-type probes (S1 to S5) encompass the region of the rpo $\beta$ gene encoding amino acids 509 to 534. Four other probes are specific for the most common mutations, D516V, H526Y, H526D, and S531L (probes R2, R4a, R4b, and R5, respectively). Hybridized PCR products were detected, and the LiPA results were evaluated as described elsewhere [43].

\subsection{Methods for the molecular epidemiology analysis of the resistant M. tuberculosis strains in Hungary}

All 68 drug-resistant Mycobacterium tuberculosis complex strains that were identified in the Hungarian Reference Laboratory for Mycobacteria at the Korányi National Institute for Tuberculosis and Respiratory Medicine in 2003 and 2004 were analyzed by IS6110 restriction fragment length polymorphism (RFLP) fingerprinting and spoligotyping. These isolates were submitted to the reference centre for susceptibility testing from all over Hungary and represented $44.2 \%$ of all drug-resistant tuberculosis cases reported in Hungary in 2003 and 2004 [21, 44].

Testing of all isolates for susceptibility to INH, RMP, EMB, and SM was carried out by the proportion method on Löwenstein-Jensen medium as described previously by Canetti et al. [40]. Conventional epidemiologic data were obtained from the database of the National Tuberculosis Surveillance System (NTSC) as described elsewhere previously [45].

IS6110 RFLP-fingerprinting was performed in line with a standardized protocol as described previously [46-48]. Spoligotyping was performed with a commercially available kit (Isogen Bioscience BV, Maarssen, The Netherlands) according to the instructions of the manufacturer [49]. The IS6110 fingerprint and spoligotype patterns of the examined strains were analyzed using Bionumerics software, version 3.5 (Applied Maths, Sint-Martens-Latem, Belgium), as described previously [46-48]. Clusters were defined as groups of patients with M. tuberculosis strains showing identical IS6110 RFLP (same number of IS6110 bands at identical positions [position tolerance, 1.3\%]) and spoligotype patterns.

The $\chi 2$ test and the Mann-Whitney $U$ test were employed to evaluate differences in demographic, epidemiologic, and drug resistance characteristics between clustered and non-clustered patients. Values of P of less than 0.05 were considered significant. 
In order to display the degree of relatedness of the isolates, a dendrogram was generated by applying the Dice coefficient and the unweighted-pair group method with arithmetic mean (UPGMA).

\subsection{Methods for the identification of the new $M$. tuberculosis clade among homeless people in Budapest}

Conventional epidemiologic data were obtained from the database of the National Tuberculosis Surveillance Centre (NTSC) as described elsewhere [45]. Since this retrospective study was using isolates from specimens that were submitted for routine diagnostic purpose, no ethical approval was required.

A total of 66 mycobacterial strains were isolated from 66 newly diagnosed homeless patients with tuberculosis in the Hungarian Reference Laboratory for Mycobacteria at Korányi Institute for Tuberculosis and Respiratory Medicine in 2002. These cases were representing $47.1 \%$ of all homeless tuberculosis cases in Budapest during the study period [50]. All cultures were identified as Mycobacterium tuberculosis complex by means of the AccuProbe culture identification test (Gen-Probe Inc., San Diego, CA) and conventional biochemical tests $[37,51]$.

Spoligotyping was performed with a commercially available kit (Isogen Bioscience BV, Maarssen, The Netherlands) according to the instructions of the manufacturer [49]. Briefly, genomic mycobacterial DNA was directly extracted from cultures by heating at $90^{\circ} \mathrm{C}$ for $30 \mathrm{~min}$. Oligonucleotides DRa and DRb were used as primers to amplify the whole DR region by polymerase chain reaction. The amplified biotinylated products were hybridized to a set of 43 immobilized oligonucleotides, each corresponding to one of the unique spacer DNA sequences within the DR locus. After hybridization, the membrane was washed, incubated with streptavidin-peroxidase conjugate (Boehringer Mannheim, Indianapolis, Ind.), and finally developed by chemiluminescence. The analysis of spoligotyping data was done in the SpolDB4 Information System which is an automated access-based labelling and matching system [52].

Extraction of mycobacterial DNA for IS6110 RFLP-fingerprinting was performed in line with a standardized protocol as described previously [46-48]. The IS6110 RFLP-fingerprinting patterns of the examined strains were analyzed and clusters were defined as described in the chapter 6.3. 
The MIRU typing was performed as described previously [53]. MIRU is a PCR-based typing method which assigns the number of tandem repeats in 12 independent loci (MIRUs) that are polymorphic in M. tuberculosis. Therefore, the MIRU type consists of a 12-number code that indicates the number of tandem repeats found for each of the MIRU loci. Clustering analysis between genotypes was performed using Bionumerics version 3.0.

Classification of $M$. tuberculosis isolates into one of the three principal genetic groups was performed on the basis of the combination of polymorphisms at codon 463 of the katG gene and at codon 95 of the gyrA gene [54]. Polymorphisms were evaluated by a real-time PCR assay with primers and probes designed for use with an Applied Biosystem 7000 realtime PCR system (Applied Biosystems, Foster City, CA) [55]. 


\section{Results}

\subsection{Evaluation of the automated non-radiometric MGIT 960 system versus the semi-automated radiometric Bactec 12B system and solid medium for isolation of Mycobacteria}

A total of 57 specimens (15.1\%) were positive for mycobacteria, of which 14 (24.6\%) were AFB smear positive and 43 (75.4\%) were AFB smear negative for the respiratory specimen used. The mycobacterial species identified were Mycobacterium tuberculosis $(\mathrm{n}=55)$, Mycobacterium avium complex $(\mathrm{n}=1)$, and Mycobacterium xenopi $(\mathrm{n}=1)$. The numbers of isolates of mycobacteria recovered by Bactec MGIT 960, Bactec 12B, and LJ medium are presented in Table 1. As a single medium, Bactec MGIT 960 recovered 53 (96.4\%) of the 55 M. tuberculosis isolates, Bactec 12B recovered 51 (92.7\%) of the 55 isolates, and LJ medium recovered 45 (81.8\%) of the 55 isolates. A statistically significant difference was demonstrated between Bactec MGIT 960 and LJ medium $(\mathrm{P}<0.05)$.

Two M. tuberculosis isolates grew in the Bactec MGIT 960 but not on the Bactec 12B or $\mathrm{LJ}$ medium. Bactec 12B or LJ medium did not detect any isolates alone. It is possible that those two isolates recovered by the Bactec MGIT 960 alone did not metabolize the $\left[{ }^{14} \mathrm{C}\right]$ palmitic acid in Bactec 12B or that the higher volume of the Bactec MGIT 960 diluted potential growth inhibitors in the specimen. Each system detected all 14 AFB smear-positive specimens, and all of these contained $M$. tuberculosis. For the AFB smear-negative specimens, the $M$. tuberculosis recovery rates were 39 (95.1\%) of 41 isolates with Bactec MGIT 960, 37 (90.2\%) of 41 isolates with Bactec 12B, and 31 (75.6\%) of 41 isolates with LJ medium. Again, a statistically significant difference was found between Bactec MGIT 960 and LJ medium $(\mathrm{P}<0.05)$. The number of non-tuberculous mycobacteria $(\mathrm{NTM})$ in this study was too low to allow a meaningful statistical comparison for this group. 
Table 1. Rates of recovery of mycobacteria and contaminants by Bactec MGIT 960, Bactec 12B, and LJ medium

No. (\%) of isolates recovered *

\begin{tabular}{lrrrr}
\cline { 2 - 5 } Medium & $\begin{array}{c}\text { All organisms } \\
\text { (\% among all } \\
\text { Mycobacteria } \\
\text { recovered) }\end{array}$ & $\begin{array}{c}\text { M. tuberculosis } \\
\text { (\% among all TB } \\
\text { recovered) }\end{array}$ & $\begin{array}{c}\text { NTM } \\
\text { (\% among all } \\
\text { NTB recovered) }\end{array}$ & $\begin{array}{c}\text { Contaminants } \\
\text { (\% among all samples } \\
\text { tested) }\end{array}$ \\
\hline Bactec MGIT 960 & $55(96.5)$ & $53(96.4)$ & $2(100)$ & $14(3.7)$ \\
Bactec 12B & $53(93.0)$ & $51(92.7)$ & $2(100)$ & $11(2.9)$ \\
LJ & $46(80.7)$ & $45(81.8)$ & $1(50)$ & $4(1.2)$ \\
\hline
\end{tabular}

* In total 377 consecutive clinical samples were tested. The total number of organisms recovered was 57, of which 55 were M. tuberculosis, 2 were NTM, and 17 were contaminants. $\kappa^{2}$ test for differences in recovery of mycobacteria: Bactec MGIT 960 versus LJ medium, $P<0.05$ (significant). $\kappa^{2}$ test for differences in recovery of $M$. tuberculosis: Bactec MGIT 960 versus $L J$ medium, $P<0.05$ (significant).

The recovery rates obtained for M. tuberculosis were 53 (96.4\%) of 55 isolates with Bactec MGIT 960 plus LJ medium and 51 (92.7\%) of 55 isolates with Bactec 12B plus LJ medium. The statistical analysis did not reveal any significant difference between the two combinations. Therefore, combination of the Bactec MGIT 960 and LJ medium could be a reliable alternative to replace the standard liquid radiometric plus solid medium combination.

The mean (range) times to detect the all M. tuberculosis isolates were 14.3 (6 to 24), 16.6 (8 to 23), and 35.8 (14 to 58) days with Bactec MGIT 960, Bactec 12B, and LJ medium, respectively. ANOVA and the Newman-Keuls test revealed statistically significant differences between Bactec MGIT 960 and LJ medium and between Bactec 12B and LJ medium ( $\mathrm{P}<0.001$ and $\mathrm{P}<0.001$, respectively). The difference in detection time between the two liquid media was not statistically significant. The mean times to detect the growth of all mycobacteria and M. tuberculosis with regard to results of acid-fast microscopy are listed in Table 2.

Table 2. Mean time to detection of all mycobacteria and M. tuberculosis in clinical specimens

Mean no. of days (range) to detection of *:

\begin{tabular}{lrrr}
\hline \multirow{2}{*}{ Medium } & Mycobacteria & \multicolumn{2}{c}{ M. tuberculosis } \\
\cline { 3 - 4 } & & $\begin{array}{c}\text { Smear } \\
\text { positive }\end{array}$ & \multicolumn{1}{c}{$\begin{array}{c}\text { Smear } \\
\text { negative }\end{array}$} \\
\hline BACTEC MGIT 960 & $13.2(6-24)$ & $12.6(8-18)$ & $15.8(6-24)$ \\
BACTEC 12B & $16.8(8-23)$ & $13.8(8-23)$ & $17.7(9-23)$ \\
LJ & $36.2(14-58)$ & $20.1(14-27)$ & $42.2(18-58)$ \\
\hline
\end{tabular}

* ANOVA, $P, 0.001$. Newman-Keuls test for differences in mean times to detection of mycobacteria and $M$. tuberculosis: BACTEC MGIT 960 versus LJ medium, $P<0.001$ (significant), BACTEC 12B versus LJ medium, $P<0.001$ (significant). 
We did not observe any false-positive cultures (instrument positive but smear and Columbia agar subculture negative) with Bactec MGIT 960 during the study. However, in eight Bactec MGIT 960 vials signalled to be positive by the instrument, the confirmatory acid-fast microscopy was negative and the presence of AFB could be detected by ZN-staining only after incubation for 3 to 4 more days.

\subsection{The $r p o \beta$ gene mutation analysis of the rifampin resistant $M$. tuberculosis strains by PCR-based DNA sequencing and reverse- hybridisation}

Of the 29 RMP-resistant isolates, only 2 (6.9\%) were resistant to RMP alone. Twentysix (89.7\%) were also resistant to INH (and thus classified as multidrug resistant), 18 (62.1\%) were also resistant to EMB, and 9 (31.0\%) were also resistant to SM (Table 3). In all, 20 of the $29(70.0 \%)$ were resistant to at least three of the four first-line drugs.

In contrast with previous reports, the frequency of occurrence of particular mutations was different in the isolates from East Hungary (see Table 4) [56-64], with 11 (37.9\%) isolates carrying the less common D516V mutation. Nine (31.0\%) isolates had an S531L mutation, and two (6.9\%) isolates had an H526D mutation. These mutations were also correctly detected in the LiPA. In addition, DNA sequencing identified a double mutation (S509T and D516V) and a deletion (deletion 522-525) that have not been reported in the literature before [65]. In these two cases, the LiPA was unable to detect the correct type of the mutation. However, it indicated the presence of the genetic alteration (Table 3). The LiPA also did not reveal the type of mutation in two additional strains with rare mutation patterns (Q513K and Q513P) (Table 3). Moreover, the test provided a false-resistant result with a pansusceptible control strain with a silent mutation (R529R) (see Table 3). 
Table 3. Resistance patterns of RMP-resistant M. tuberculosis isolates from Hungary by the proportion method, the line probe assay, and DNA sequencing of the 81-bp region of the rpo $\beta$ gene ${ }^{\text {a }}$

\begin{tabular}{|c|c|c|c|c|c|c|c|c|c|c|c|c|c|c|c|c|}
\hline \multirow{3}{*}{ Strain } & \multicolumn{14}{|c|}{ Result by method: } & \multirow{2}{*}{\multicolumn{2}{|c|}{ Mutation }} \\
\hline & \multicolumn{4}{|c|}{ Proportion method } & \multicolumn{10}{|c|}{ LiPA } & & \\
\hline & SM & $\mathrm{INH}$ & RMP & EMB & TB-P & $\mathrm{S} 1$ & $\$ 2$ & S3 & S4 & S5 & $\mathrm{R} 2$ & R4a & $\mathrm{R} 4 \mathrm{~b}$ & R5 & LiPA & Seq. \\
\hline 1 & S & $\mathrm{R}$ & $\mathrm{R}$ & $\mathrm{R}$ & + & + & - & t & + & + & + & - & - & - & D $516 \mathrm{~V}$ & D516V \\
\hline 2 & S & $\mathrm{R}$ & $\mathrm{R}$ & $S$ & + & t & t & t & + & - & - & - & - & + & S531L & S531L \\
\hline 3 & $\mathrm{~S}$ & $\mathrm{R}$ & $\mathrm{R}$ & $\mathrm{R}$ & + & t & - & t & + & + & + & - & - & - & D516V & D516V \\
\hline 4 & S & $\mathrm{R}$ & $\mathrm{R}$ & $S$ & + & t & t & + & + & - & - & - & - & + & S531L & S531L \\
\hline 5 & $\mathrm{R}$ & S & $\mathrm{R}$ & $S$ & + & t & t & t & + & - & - & - & - & t & S531L & S531L \\
\hline 6 & S & S & $\mathrm{R}$ & $S$ & + & + & t & - & - & $+1-$ & - & - & - & - & UDR & Deletion $522-525$ \\
\hline 7 & S & $\mathrm{R}$ & $\mathrm{R}$ & $\mathrm{R}$ & + & t & - & t & + & + & + & - & - & - & D516V & D516V \\
\hline 8 & S & $\mathrm{R}$ & $\mathrm{R}$ & $\mathrm{R}$ & + & t & - & t & + & t & + & - & - & - & D $516 \mathrm{~V}$ & D516V \\
\hline 9 & $\mathrm{R}$ & $\mathrm{R}$ & $\mathrm{R}$ & $S$ & + & + & t & t & - & + & - & - & + & - & $\mathrm{H} 526 \mathrm{D}$ & $\mathrm{H} 526 \mathrm{D}$ \\
\hline 10 & S & $\mathrm{R}$ & $\mathrm{R}$ & $\mathrm{R}$ & + & t & - & t & + & + & + & - & - & - & D $516 \mathrm{~V}$ & D516V \\
\hline 11 & $\mathrm{R}$ & $\mathrm{R}$ & $\mathrm{R}$ & $\mathrm{R}$ & + & t & - & t & + & + & + & - & - & - & D516V & D516V \\
\hline 12 & $\mathrm{R}$ & $\mathrm{R}$ & $\mathrm{R}$ & $\mathrm{R}$ & + & t & - & t & + & + & + & - & - & - & D516V & D $516 \mathrm{~V}$ \\
\hline 13 & S & $\mathrm{R}$ & $\mathrm{R}$ & $\mathrm{R}$ & + & t & t & t & + & - & - & - & - & t & S531L & S531L \\
\hline 14 & $\mathrm{R}$ & $\mathrm{R}$ & $\mathrm{R}$ & $\mathrm{R}$ & + & + & + & t & + & - & - & - & - & + & S531L & S531L \\
\hline 15 & S & $\mathrm{R}$ & $\mathrm{R}$ & $\mathrm{R}$ & + & t & - & t & + & t & + & - & - & - & D $516 \mathrm{~V}$ & D $516 \mathrm{~V}$ \\
\hline 16 & S & $\mathrm{R}$ & $\mathrm{R}$ & $\mathrm{R}$ & + & t & - & + & + & + & + & - & - & - & D $516 \mathrm{~V}$ & S509T/D516V \\
\hline 17 & S & $\mathrm{R}$ & $\mathrm{R}$ & $S$ & + & - & t & t & + & + & - & - & - & - & UDR & Q513K \\
\hline 18 & S & $\mathrm{R}$ & $\mathrm{R}$ & $\mathrm{R}$ & + & + & t & t & + & + & - & - & - & - & UD & WT \\
\hline 19 & S & $\mathrm{R}$ & $\mathrm{R}$ & $\mathrm{R}$ & + & t & t & t & + & - & - & - & - & + & S531L & S531L \\
\hline 20 & $\mathrm{R}$ & $\mathrm{R}$ & $\mathrm{R}$ & $S$ & + & $+/-$ & t & t & + & t & - & - & - & - & UDR & Q513P \\
\hline 21 & S & $\mathrm{R}$ & $\mathrm{R}$ & $S$ & + & + & + & + & + & - & - & - & - & + & S531L & S531L \\
\hline 22 & S & $\mathrm{R}$ & $\mathrm{R}$ & $S$ & + & + & t & t & - & + & - & - & + & - & $\mathrm{H} 526 \mathrm{D}$ & H526D \\
\hline 23 & S & $\mathrm{R}$ & $\mathrm{R}$ & $\mathrm{R}$ & + & t & - & t & + & + & + & - & - & - & D516V & D516V \\
\hline 24 & S & $\mathrm{R}$ & $\mathrm{R}$ & $\mathrm{R}$ & + & + & - & t & + & + & + & - & - & - & D516V & D516V \\
\hline 25 & S & $\mathrm{R}$ & $\mathrm{R}$ & $S$ & + & + & - & t & + & + & + & - & - & - & D516V & D516V \\
\hline 26 & S & $\mathrm{S}$ & $\mathrm{R}$ & $S$ & + & t & t & + & + & - & - & - & - & + & S531L & S531L \\
\hline 27 & $\mathrm{R}$ & $\mathrm{R}$ & $\mathrm{R}$ & $\mathrm{R}$ & + & t & + & + & + & - & - & - & - & + & S531L & S531L \\
\hline 28 & $\mathrm{R}$ & $\mathrm{R}$ & $\mathrm{R}$ & $\mathrm{R}$ & + & t & + & + & + & + & - & - & - & - & WT & $\mathrm{WT}^{b}$ \\
\hline 29 & $\mathrm{R}$ & $\mathrm{R}$ & $\mathrm{R}$ & $\mathrm{R}$ & + & + & + & + & + & + & - & - & - & - & WT & WT \\
\hline Co. 1 & S & S & $S$ & $S$ & + & t & + & + & - & - & - & - & - & - & UDR & R529R \\
\hline Co. 2 & S & S & $\mathrm{S}$ & $\mathrm{S}$ & + & t & + & + & + & + & - & - & - & - & WT & WT \\
\hline Co. 3 & S & S & S & S & + & t & + & + & + & + & - & - & - & - & WT & WT \\
\hline Co. 4 & $\mathrm{~S}$ & S & $\mathrm{S}$ & $\mathrm{S}$ & + & t & + & + & + & + & - & - & - & - & WT & WT \\
\hline Co. 5 & S & S & $\mathrm{S}$ & $S$ & + & t & + & + & + & + & - & - & - & - & WT & WT \\
\hline Co. 6 & S & S & $\mathrm{S}$ & $S$ & + & + & t & t & + & + & - & - & - & - & WT & WT \\
\hline $\mathrm{H} 37 \mathrm{Rv}$ & S & S & $S$ & $S$ & + & + & + & + & + & + & - & - & - & - & WT & WT \\
\hline
\end{tabular}

a. Abbreviations: Co., control; Seq., DNA sequencing of the hypervariable region; S, susceptible; R, resistant; +, positive; -, negative; +/-, indeterminate; UD, undetermined; UDR, undetermined resistant; WT, wild type. Probes R2, R4a, R4b, and R5 are specific for mutations D516V, H526Y, H526D, and S531L, respectively.

b. A V146F mutation was found in this isolate. 
Table 4. Frequency of mutations in RMP-resistant $M$. tuberculosis isolates from different geographic regions

\begin{tabular}{|c|c|c|c|c|c|c|c|c|c|}
\hline \multirow[b]{2}{*}{ Mutated codon(s) } & \multicolumn{9}{|c|}{$\%$ Mutations in geographic region: } \\
\hline & $\begin{array}{c}\text { Africa }^{a} \\
\text { (reference 22; } \\
n=105 \text { ) }\end{array}$ & $\begin{array}{c}\text { Asia }^{b} \\
\text { (reference 8; } \\
n=77 \text { ) }\end{array}$ & $\begin{array}{c}\text { Australia } \\
\text { (reference 31; } \\
n=33 \text { ) }\end{array}$ & $\begin{array}{c}\text { Brazil }^{c} \\
\text { (reference 27; } \\
n=82 \text { ) }\end{array}$ & $\begin{array}{l}\text { Germany }^{d} \\
\text { (reference } 20 \\
n=36 \text { ) }\end{array}$ & $\begin{array}{c}\text { Greece }^{e} \\
\text { (reference 13; } \\
n=17 \text { ) }\end{array}$ & $\begin{array}{c}\text { Italy } \\
\text { (reference } 18 ; \\
n=37 \text { ) }\end{array}$ & $\begin{array}{c}\text { United States } \\
\text { (reference 30; } \\
n=61 \text { ) }\end{array}$ & $\begin{array}{r}\text { Hungary } f \\
(n=29)\end{array}$ \\
\hline 572 & & & 3.0 & & & & & & \\
\hline 533 & 3.0 & & & 1.2 & & & & 3.3 & \\
\hline 531 & 53.5 & 46.8 & 48.5 & 53.7 & 36.1 & 47.1 & 56.7 & 34.4 & 31.0 \\
\hline 526 & 21.2 & 18.2 & 30.3 & 20.6 & 22.2 & 17.7 & 29.7 & 39.3 & 6.8 \\
\hline 522 & $10^{g}$ & 1.3 & 6.1 & 2.4 & & & & 1.6 & \\
\hline 521 & & & & & & & & 1.6 & \\
\hline 518 & 1.0 & & & & & & & 1.6 & \\
\hline 516 & 13.1 & 16.9 & 9.1 & 7.4 & 2.8 & 5.9 & 2.7 & 6.6 & 37.9 \\
\hline 514 & & 2.6 & & & & & & 1.6 & \\
\hline 513 & 3.0 & 6.5 & & 1.2 & & & & & 6.8 \\
\hline 511 & 2.0 & 1.3 & & 1.2 & & & & & \\
\hline 508 & & & & & & 17.7 & & & \\
\hline 505 & & & & & & 5.9 & & & \\
\hline 504 & 2.0 & & & & & & & & \\
\hline 531 and 526 & & & & 1.2 & 2.8 & 5.9 & 2.7 & & \\
\hline No mutation & 14.1 & 6.5 & 3.0 & 3.7 & 33.3 & & & 8.2 & $10.3^{h}$ \\
\hline
\end{tabular}

a. South Africa $(n=87)$, Sierra Leone and Uganda $(n=16)$, and Namibia $(n=2)$.

b. Bangladesh $(n=2)$, India $(n=4)$, Indonesia $(n=1)$, Malaysia $(n=14)$, Myanmar $(n=15)$, Nepal $(n=2)$, the Philippines $(n=4)$, Thailand $(n=14)$, and Yemen $(n=21)$.

c. Less common mutations (1.2\% each) were as follows: 531 and 514; 516 and 511; 515 and 511; 526 and 531; 524, 525, and 526 and deletion 527; deletion 515, 516, 517, and 518; 513 and deletion 514, 515, and 516.

d. A rare (2.8\%) double mutation was detected at codons 526 and 511.

e. $\quad$ Rare (2.7\% each) triple mutations were detected at codons 553, 541, and 526; 525, 523, and 516; and 516, 512, and 511.

f. A deletion (522-525) that has not been reported previously was detected in one strain (3.4\%).

g. Silent mutation.

h. In one of these three strains, an N-terminal V146F mutation was detected.

\subsection{Molecular epidemiology of resistant M. tuberculosis strains in Hungary}

In order to display the degree of relatedness of the resistant isolates tested during our study, a dendrogram was generated by applying the Dice coefficient and the UPGMA (Figure 4). Thirty-one isolates (45.6\%) showed unique fingerprint patterns, while 37 isolates (54.4\%) had an IS6110 RFLP pattern and a spoligotyping pattern identical to those of at least one other isolate and were thus grouped into 12 different clusters (Figure 4). Eleven (16.2\%) patients belonged to the largest cluster (cluster 2), 12 (17.6\%) patients belonged to four smaller clusters with 3 patients each (clusters 1, 4, 10, and 12), and 14 (20.6\%) patients belonged to seven clusters with 2 patients each (clusters 3, 5, 6, 7, 8, 9, and 11) (Figure 4). 

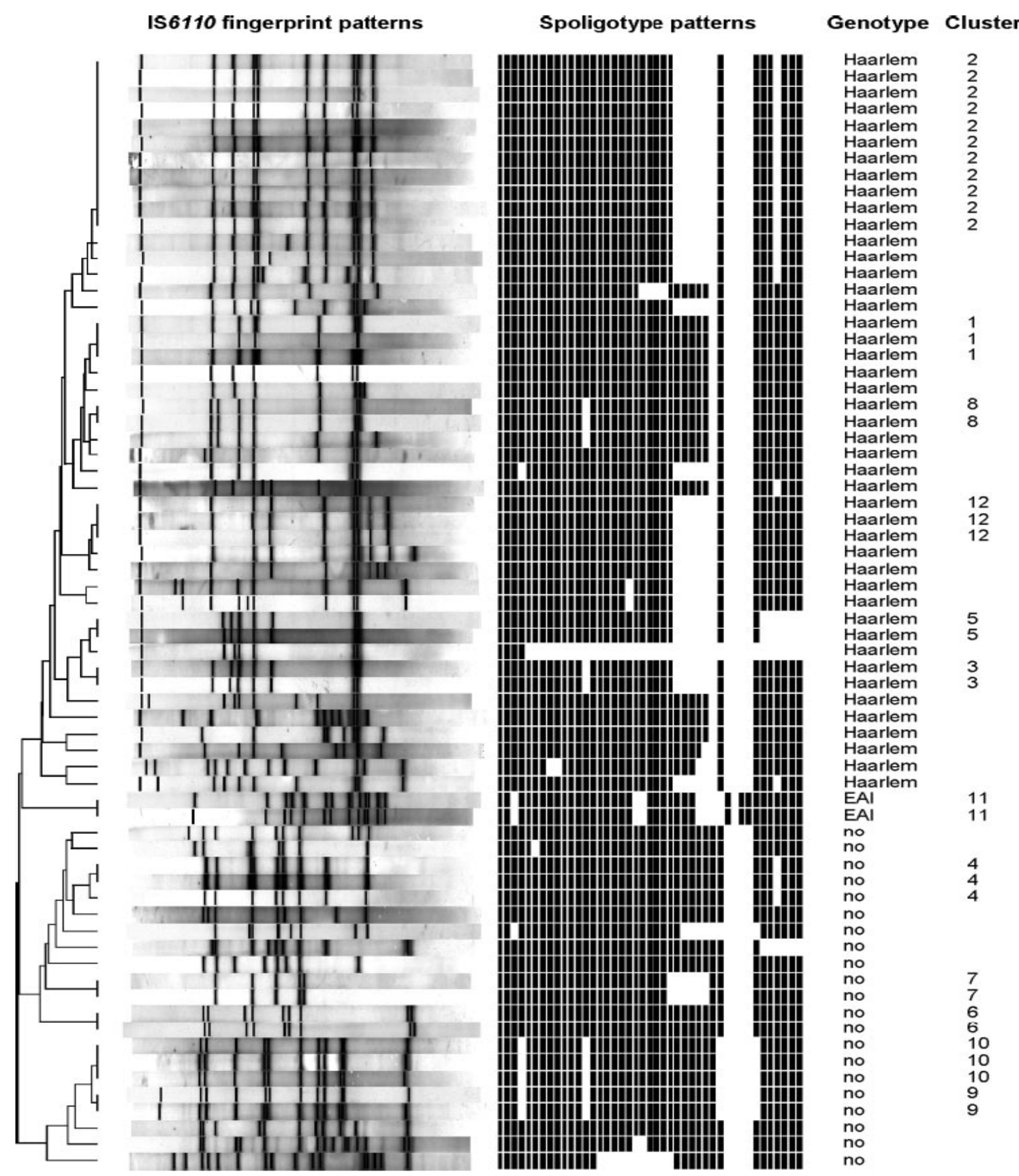

Figure 4. IS6110 fingerprint and spoligotyping patterns of the 68 patients with drug-resistant tuberculosis. EAI, East African-Indian.

Analysis of the spoligotyping patterns revealed that a remarkably high number (45 [66.2\%]) of patients were infected with strains of the Haarlem genotype, while two (2.9\%) patients were infected with an East African-Indian genotype strain, and no classification of major genotypes was possible for 21 (30.9\%) isolates (Figure 4). It is 
noteworthy that none of the patients were infected by strains of the Beijing genotype during this period in Hungary.

The characteristics of the 68 patients with drug-resistant tuberculosis are summarized in Table 5. All the patients were human immunodeficiency virus-negative. Statistical analysis did not find any significant difference between clustered and non-clustered patients with regard to their demographic characteristics or their medical and social risk factors.

Table 5. Characteristics of the 68 clustered and non-clustered patients from Hungary with drug resistant-tuberculosis

\begin{tabular}{|c|c|c|c|c|}
\hline \multirow[b]{2}{*}{ Characteristic } & \multicolumn{3}{|c|}{ No. (\%) of specimens } & \multirow[b]{2}{*}{ P value $^{\mathrm{a}}$} \\
\hline & $\begin{array}{c}\text { Clustered } \\
(\mathbf{n}=37)\end{array}$ & $\begin{array}{c}\text { Nonclustered } \\
(n=31)\end{array}$ & $\begin{array}{c}\text { Total } \\
(n=68)\end{array}$ & \\
\hline Gender & & & & 0.4951 \\
\hline Male & $30(81.1)$ & $23(74.2)$ & $53(77.9)$ & \\
\hline Female & 7 (18.9) & $8(25.8)$ & $15(22.1)$ & \\
\hline Mean age (yr) & 48.7 & 53.7 & 50.9 & $0.1360^{\mathrm{b}}$ \\
\hline Male & 47.5 & 51.9 & 49.4 & \\
\hline Female & 53.9 & 58.9 & 56.5 & \\
\hline Previous treatment & & & & 0.9637 \\
\hline No & $30(81.1)$ & $25(80.6)$ & 55 (80.9) & \\
\hline Yes & 7 (18.9) & $6(19.4)$ & $13(19.1)$ & \\
\hline AFB microscopy & & & & 0.5148 \\
\hline Positive & $21(56.8)$ & $20(64.5)$ & $41(60.3)$ & \\
\hline Negative & $16(43.2)$ & $11(35.5)$ & 27 (39.7) & \\
\hline Alcohol abuse & $11(29.7)$ & $9(29.0)$ & $20(29.4)$ & 0.9499 \\
\hline Homeless & $9(24.3)$ & $4(12.9)$ & $13(19.1)$ & 0.2329 \\
\hline Contact & $1(2.7)$ & $1(3.2)$ & $2(2.9)$ & 0.8988 \\
\hline Foreign origin & $1(2.7)$ & $1(3.2)$ & $2(2.9)$ & 0.8988 \\
\hline $\begin{array}{l}\text { Resident of congregate } \\
\text { facility }\end{array}$ & $1(2.7)$ & 0 & $1(1.5)$ & 0.3565 \\
\hline
\end{tabular}

${ }^{\text {a }}$ Chi-square test.

${ }^{\mathrm{b}}$ Mann-Whitney U test.

A review of the database of the NTSC revealed that many of the related isolates were from patients located in similar geographic regions. For example, of the 11 patients of cluster 2 (the largest cluster), 5 (2 homeless) were from neighbouring downtown districts of 
Budapest, and 3 were residents of two adjacent, high-incidence, northeast counties (Heves and Nógrád Counties). Similar coincidences of genotype and geographic location were observed for patients in all other clusters: two of the three patient in cluster 1, both patients in cluster 3 , all three patients in cluster 4 , both patients in cluster 5 , both patients in cluster 6 , both patients in cluster 7 , both patients in cluster 8 , two of the three patients in cluster 10 , both patients in cluster 11, and two of the three patients in cluster 12 were from the same geographic location. Three patients with MDR tuberculosis were not reported to the NTSC.

Results of susceptibility testing of the 68 resistant isolates by the proportion method are presented in Table 6.

Table 6. Drug susceptibility patterns of M. tuberculosis strains isolated from the 68 clustered and non-clustered patients from Hungary with drug-resistant tuberculosis

\begin{tabular}{lcccc}
\hline \multirow{1}{*}{ Characteristic } & \multicolumn{3}{c}{ No. (\%) of specimens } & \multirow{2}{*}{ P value } \\
\cline { 2 - 4 } & $\begin{array}{c}\text { Clustered } \\
(\mathbf{n}=\mathbf{3 7 )}\end{array}$ & $\begin{array}{c}\text { Nonclustered } \\
(\mathbf{n}=\mathbf{3 1})\end{array}$ & $\begin{array}{c}\text { Total } \\
(\mathbf{n}=\mathbf{6 8})\end{array}$ & \\
\hline Mono-INH resistant & $6(16.2)$ & $7(22.6)$ & $13(19.1)$ & 0.5062 \\
Mono-RMP resistant & $1(2.7)$ & $1(3.2)$ & $2(2.9)$ & 0.8988 \\
Mono-SM resistant & $2(5.4)$ & $7(22.6)$ & $9(13.2)$ & 0.0374 \\
Polyresistant (not MDR) & $7(18.9)$ & $9(29.0)$ & $16(23.5)$ & 0.3275 \\
MDR & $21(56.8)$ & $7(22.6)$ & $28(41.2)$ & 0.0043 \\
\hline
\end{tabular}

Notably, 21 (75.0\%) of the MDR strains were clustered. Comparison of susceptibility patterns of clustered and non-clustered patients revealed a statistically significant difference for mono-SM-resistant $(\mathrm{P}=0.0374)$ and MDR $(\mathrm{P}=0.0043)$ patients. In addition, in several clusters, a good correlation was found between the IS6110 fingerprints and the drug susceptibility patterns, implying a close relationship between these strains (data not shown).

\subsection{Identification of a new $M$. tuberculosis strain among homeless people in Budapest}

The study population of the homeless people consisted of 61 men (92.4\%) and 5 women (7.6\%). All were born in Hungary. The patients were from 31 to 73 years old, and the mean age was 47.8 years (men: 48.2 years; women: 44.4 years). All patients were HIV 
negative. The spoligotyping patterns of all strains were analyzed by using the SpolDB4 database, a follow-up of the SpoIDB3 project [66, 67]. Fifty-one (78\%) strains could be classified into 7 shared types [ST50 $(\mathrm{n}=17)$, ST1773 $(\mathrm{n}=10)$, ST53 $(\mathrm{n}=8)$, ST52 $(\mathrm{n}=6)$, ST49 (n = 4), ST62 (n = 3), ST1771 (n = 3)] while 15 strains remained orphan (10 pseudoorphans that were unique in this study but were already found in the database and 5 true orphans). None of the patients were infected by strains of the Beijing genotype also in this population. Further analysis of the database revealed that strains belonging to share type ST1773 (777777547760471, octal designation) have a unique spoligotyping pattern (Figure 5) that may well define a local lineage.

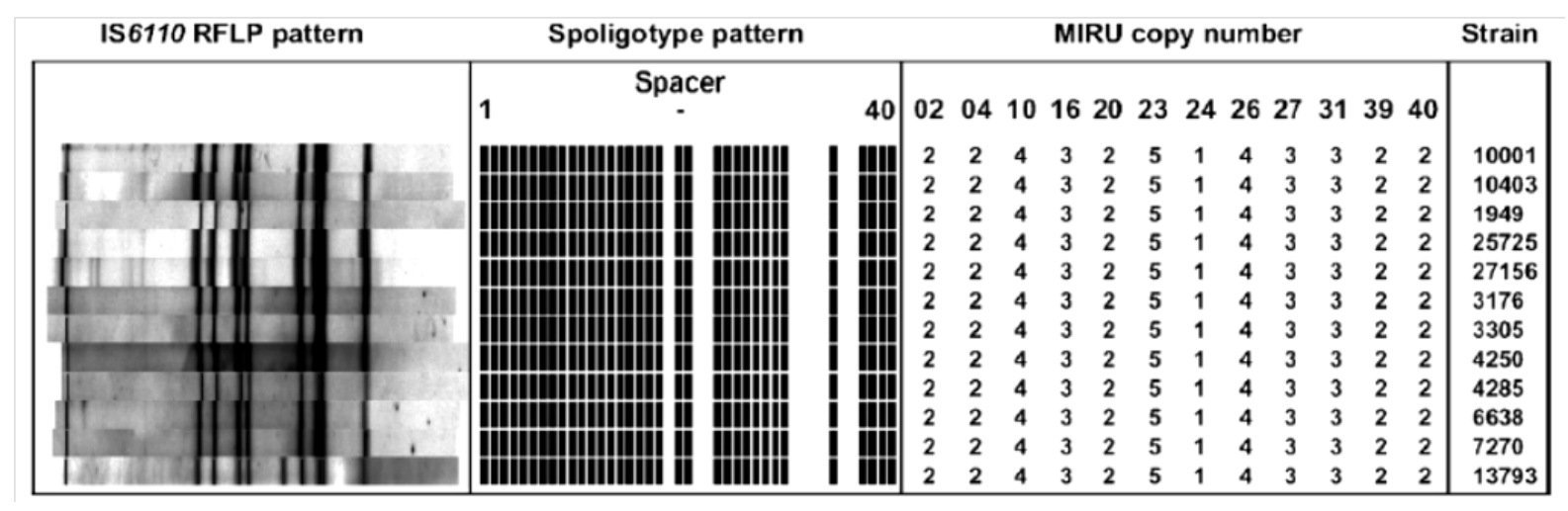

Figure 5. IS6110 fingerprint, spoligotype and mycobacterial interspred repetitive unit typing patterns of the 12 patients belonging to the Budapest cluster

The identified pattern is only one spacer different from the pattern of ST370 (777777747760471) with 18 isolates from Sweden $(n=1)$, Estonia $(n=1)$, USA $(n=2)$, Austria ( $n=5)$, Belgium ( $n=1)$, Tunisia $(n=1)$, and Brazil $(n=6)$. It is also very close to pattern ST65 (777777777760471) which was first described in Austria and could be a potential ancestor.

By IS6110 RFLP-fingerprinting analysis 47 (71.2\%) isolates could be classified into 11 clusters. Thirty-four (51.5\%) patients belonged to 5 major clusters (size from 4 to 11 individuals), and $13(19.7 \%)$ to 6 smaller clusters with 3 or less individuals. The IS6110 RFLP-fingerprinting analysis of members of shared type ST1773 showed that 11 of the 12 strains belonged to the same IS6110 cluster. Interestingly, there was only 1 band difference between the one IS6110 RFLP non-clustered strain and the 11 strains in the largest cluster (Figure 5). In order to characterize more accurately the identified local lineage MIRU typing 
was performed on these 12 isolates. The MIRU analysis showed identical patterns (pattern 224325143322) for all 12 strains (Figure 5). When introducing the MIRU findings into the international MIRU provisional web page (http://www.ibl.fr/mirus/mirus.html) the Budapest pattern showed matches only with two other strains of the database. When introduced into the SITVIT database (Institut Pasteur de Guadeloupe, unpublished results), which contains both spoligotyping and MIRU alleles from all-over the world, the Budapest spoligo-MIRU combined pattern did not match with any other strain of the database. However, 20 clinical isolates from the USA and Belgium in the SITVIT database with spoligotyping patterns ST91, ST119, or ST197, all belonging to the X genotype family of strains, shared the same pattern of the Budapest clade, reflecting a genetic convergence on or a genetic divergence from this MIRU-type. Analysis of polymorphisms at codon 463 of the katG and at codon 95 of the gyrA in all 12 isolates of share type ST1773 showed that they belong to the Principal Genetic Group 2, which suggests that this clone could be deeply rooted in the M. tuberculosis global phylogenetical tree [68, 69].

The retrospective review of the database of the NTSC revealed that all 12 patients of share type ST1773 were from the $8^{\text {th }}$ District of Budapest (one of the poorest districts with the highest tuberculosis incidence). Further investigation of the records found that $2(16.6 \%)$ of the 12 patients were residents of the same homeless shelter. 


\section{Discussion}

The use of the radiometric Bactec 460 TB broth-based system (Becton Dickinson Diagnostic Instrument Systems, Sparks, Md.) considerably improves the recovery of and decreases the time required to detect mycobacteria; however, this procedure is still laborintensive and requires attention to special safety and regulatory issues regarding radioisotopes [32]. Previous reports have demonstrated that the manual Bactec MGIT (BBL Becton Dickinson Microbiology Systems, Cockeysville, Md.) and MB Redox (Biotest AG, Dreieich, Germany) systems are suitable non-radiometric alternatives to Bactec 12B. However, these methods still require manual processing and are best suited for laboratories which cannot afford an automated system due to the low number of processed specimens.

According to our results, the automated Bactec MGIT 960 system displayed a rate of recovery (96.4\%) of $M$. tuberculosis higher than those previously reported for the manual Bactec MGIT, i.e., 89.4\% by Pfyffer et al. [70], 81.3\% by Somoskövi and Magyar [71], and 85\% by Piersimoni et al. [72], and also higher than those previously reported for the same walk-away system, i.e., $77 \%$ by Hanna et al. [73] and $88 \%$ by Tortoli et al. [74]. The automated Bactec MGIT 960 was also more efficient than the similarly fully automated systems (sensitivity, 85.3 and 89\%) [75, 76] and showed a comparable sensitivity to one other system (96\%) [77].

The lower rate of recovery observed by Hanna et al. and Tortoli et al. may have been due to a higher contamination rate with the automated Bactec MGIT 960. Hanna et al. found that after removal of the contaminated cohorts from their analysis, the sensitivity of the automated Bactec MGIT 960 increased from 77 to 86\% [73]. Contamination did not cause a serious problem in our study. The rates of contamination were $3.7 \%, 2.9 \%$, and $1.2 \%$ for the automated Bactec MGIT 960, Bactec 12B, and LJ medium, respectively (Table 1). We found that the rate of contamination with the automated Bactec MGIT 960 was lower than the values reported previously for other semi-automated broth-based systems, but was in line with the rates of contamination observed with the manual Bactec MGIT. However, the concentration of $\mathrm{NaOH}$ used in the other semi-automated system studies was lower (2\%) than that used both in the present study (4\%) and in the manual Bactec MGIT studies (3\%). Moreover, the delay between specimen collection and processing due to longer specimen shipment times at some sites (i.e., 2 to 5 days at one of the test sites in the study by Hanna et 
al.) and the different patient population may also explain these discrepancies [70, 73]. Our laboratory usually receives the majority of samples within 30 min after collection. This rapid delivery could impact both the contamination rate and the rate of recovery.

It is generally recommended that the solid medium should not be used alone, but be used in combination with a liquid-based culture system to increase the sensitivity of cultivation for mycobacteria [32, 37, 70]. This combination is considered to be the "gold standard.” Recovery rates were also compared when each liquid medium was combined with LJ medium. The rate of recovery of $M$. tuberculosis found in our study for the automated Bactec MGIT 960 plus solid medium was in line with the findings of 92\% reported by Pfyffer et al. [70], 94.6\% reported by Somoskövi and Magyar [71], and 92\% reported by Piersimoni et al. [72] for the manual Bactec MGIT plus solid medium; 94\% reported by Woods et al. [76] and 96.1\% reported by Tortoli et al. [75] for ESP II plus solid medium; and 97\% reported by Hanna et al. [73] and 94\% reported by Tortoli et al. [74] for the automated Bactec MGIT 960 plus solid medium.

The times to detection of $M$. tuberculosis from AFB smear-positive specimens were comparable for the automated Bactec MGIT 960 and Bactec 12B (12.6 versus 13.8 days). The time to detection with the automated Bactec MGIT 960 was shorter than the time of 15.3 days reported by Piersimoni et al. [72] for the manual Bactec MGIT but was longer than the time of 9.9 days observed by Pfyffer et al. [70] and the 7.2 days reported by Somoskövi and Magyar [71], both for the manual Bactec MGIT, and the 10.6 days reported by Hanna et al. [73] and the 12.5 days reported by Tortoli et al. [74] for the automated Bactec MGIT 960. In comparison with other semi-automated systems, the time to detection of M. tuberculosis with the automated Bactec MGIT 960 in this study was lower than the 14.5 days found by Woods et al. [76] for ESP II but was higher than the 10.3 days given by Benjamin et al. [77] for $\mathrm{MB} / \mathrm{BacT}$. However, the proportion of AFB smear-positive specimens was not exactly the same in all of the studies.

In our study, the mean time to detection of M. tuberculosis in AFB smear-negative specimens was slightly shorter with the automated Bactec MGIT 960 than with Bactec 12B (15.8 versus 17.2 days). Also, the time to detection with the automated Bactec MGIT 960 was shorter than those reported by Hanna et al. (18.1 days) [73], Tortoli et al. (19.6 days) [74], Pfyffer et al. (20.3 days) [70], Somoskövi and Magyar (19.1 days) [71], and Piersimoni et al. (22.4 days) [72] for either the automated Bactec MGIT 960 or the manual Bactec 
MGIT. The mean time to detection of M. tuberculosis in AFB smear-negative specimens with the automated Bactec MGIT 960 was also much shorter than those reported for ESP II and MB/BacT, i.e., 18.9 days by Woods et al. [76] and 20.1 days by Benjamin et al. [77]. Our results indicate that the automated Bactec MGIT 960 system may be much faster for the recovery of $M$. tuberculosis from AFB smear-negative specimens than the manual Bactec MGIT method and the similarly automated broth-based systems.

Mycobacterium tuberculosis and other members of the $M$ tuberculosis complex use several strategies to resist the action of antimicrobial agents. First, the mycobacterial cell is surrounded by a specialized, highly hydrophobic cell wall that results in decreased permeability to many compounds. Active drug efflux systems and degrading or inactivating enzymes, and the genes that are associated with these functions, have been found in $M$ tuberculosis [43, 78]. However, genetic studies have shown that resistance of $M$ tuberculosis to anti-mycobacterial drugs is the consequence of spontaneous mutations in genes that encode either the target of the drug, or enzymes that are involved in drug activation. Resistance-associated point mutations, deletions, or insertions have been described for all first-line drugs (INH, RMP, PZA, EMB, and SM), and for several second-line and newer drugs (ethionamide, fluoroquinolones, macrolides, nitroimidazopyrans) [42, 56, 79, 80]. However, no single genetic alteration has yet been found that results in the MDR phenotype (defined as resistance at least to INH and RMP). Rather, MDR develops by sequential acquisition of mutations at different loci, usually because of inappropriate patient treatment. Because MDR strains are the result of cumulative mutations, growth of M. tuberculosis can successfully be controlled in the host by concomitant treatment with more than one drug. Thus, treatment regimens that consist of three to four drugs are used routinely to treat patients with tuberculosis.

The LiPA has been reported to be an easy-to-use test for the rapid detection of RMP resistance. The test is available in a kit format and, therefore, especially useful for routine work in clinical laboratories that are not capable of carrying out DNA sequencing [43, 81]. In the present study, LiPA was able to detect a genetic alteration in 26 (89.7\%) of the 29 rifampin resistant strains and to identify the particular mutation in 22 strains (75.9\%) (Table 3). 
The LiPA can provide the type of mutation for only the four most common mutations of the rро $\beta$ gene (S531L, H526Y, H526D, and D516V), while in cases of other mutations it indicates only the presence of a genetic alteration. Our findings suggest that in a geographic area such as East Hungary where less common or novel mutations of the rpo $\beta$ gene occur more frequently, the interpretation of the LiPA results may be more difficult. In such an environment, the characterization of the type of the mutation (and the resultant amino acid change) by DNA sequencing is indispensable in order to avoid the report of any false RMP resistance results.

Although more than $96 \%$ of the RMP-resistant strains have a mutation within the 81-bp core region of the $г$ $р о \beta$ gene, a recent study revealed that a mutation associated with RMP resistance can also occur in other regions of the gene, although less frequently [42]. The present study revealed three RMP resistant isolates where neither the DNA sequencing of the 81-bp region nor the LiPA detected any mutations (Table 3). However, the DNA sequencing for the detection of the V146F mutation was positive in one of these three isolates (strain 28, Table 3). All the other study isolates had the wild-type sequence in this region. The two isolates with no mutations in either region indicate that at present the confirmation of molecular results by conventional tests is still warranted. In addition, since the routinely applied DNA sequencing methods usually examine only the 81-bp region of the rpo $\beta$ gene [65], in cases where resistance is demonstrated in conventional susceptibility testing but no mutation is found we also suggest screening for the V146F mutation. If this assay also fails to detect a mutation, then other rare mutations of the rpo $\beta$ gene, heteroresistance (a mixture of susceptible and resistant strains), or, less likely, another mechanism of resistance may be involved [42, 57, 82].

The present study is the first to provide a molecular epidemiological insight into the patterns and transmission dynamics of drug-resistant tuberculosis in Hungary. The strains that were included in the study exhibit a medium degree of DNA polymorphism (43 different RFLP patterns in 68 isolates). Since strain diversity is inversely associated with the incidence of the disease, this observation may indicate that the incidence of drug-resistant tuberculosis is actually underestimated by presently available conventional epidemiologic data [83]. The rate of clustered cases (54.4\%) was much higher than the rate of clustered drug-resistant 
cases observed in studies performed in Poland (38.9\%), Belgrade, Central Serbia (43.5\%), and Germany (33\%), while it was lower than that in Estonia (67.2\%) [2, 47, 84, 85]. The high rate of clustered cases and the high rate (81.1\%) of new cases among clustered cases indicate that a significant portion of the drug-resistant cases resulted from recent transmission. This result and the fact that $75 \%$ of the MDR cases were also clustered show that the monitoring and control of drug-resistant tuberculosis is rather inadequate in Hungary (Table 6). The rate of clustered MDR cases was lower in Poland (50.7\%) and Germany (49.4\%), was similar in Belgrade (70\%), and was higher in Estonia (95.8\%) [45, 46, 84-87].

Although statistical analysis did not reveal any statistically significant association between clustering and the various demographic and epidemiologic characteristics, it is noteworthy that nearly one-third of the clustered patients were alcohol abusers, and almost one-quarter of them were homeless (Table 5). Moreover, since $28.6 \%$ of the MDR cases were homeless and $61 \%$ of the homeless cases were infected by MDR strains, it is clear that, as in other countries (The Netherlands and France), homelessness plays a major role in the transmission of drug-resistant tuberculosis in the capital, where all these cases were found $[48,88]$.

Since contact tracing in Hungary is focused mainly on transmission between close family contacts of diseased individuals, the present study could not determine relationships among all clustered patients. However, the overall correlation of residence and drug resistance profile of patients with clustering strongly supports the close relationship of the strains within a particular cluster.

Another troubling finding of this study was that 3 (10.7\%) of the 28 MDR cases were not reported to the NTSC. According to the NTSC, in 2003 and 2004, less than $40 \%$ of the newly diagnosed tuberculosis patients received the mandatory four-drug regimen from their physicians [21, 44]. This non-adherence of clinicians to national regulations could also contribute to the increase and transmission of drug-resistant tuberculosis. As the number of patients with drug-resistant tuberculosis in a particular hospital can be low, the lack of familiarity with treatment of patients with drug-resistant tuberculosis might also worsen the situation. Indeed, the importance of this problem was underlined by a recent survey conducted in France. That study revealed a 59\% treatment failure rate for 51 patients with drug-resistant tuberculosis that were treated in a total of 42 different clinical sites, only 35 of which were managed by a respiratory disease specialist [89]. 
Some M. tuberculosis genotypes, like the W-Beijing and Haarlem families, received special clinical and public health attention because of their greater ability to be transmitted $[66,90]$. Beijing strains showed a high prevalence in Russia and Estonia (30 to 50\% of all cases), while the Haarlem genotype is more prevalent in northern Europe (20\%) [84, 91]. Analysis of the spoligotyping patterns of the strains revealed that a remarkably high number (66.2\%) of the patients were infected by strains of the Haarlem genotype, while none of the patients were infected by the Beijing genotype (Figure 4). It is noteworthy that the presence of the Beijing genotype was observed in every European country from which genotyping results are available [66]. These data, and the fact that all but two patients were Hungarian, indicate that in contrast to other European Community countries (i.e., Germany, The Netherlands, and Poland), drug-resistant tuberculosis in Hungary is the result of active incountry circulation of historical clones with European descent (Haarlem family) and not the result of the importation of strains (i.e., Beijing family) from neighbouring countries (Romania and former republics of the Soviet Union) with a high rate of Beijing genotype and/or drug-resistant tuberculosis cases.

The incidence of tuberculosis among the homeless shows an alarming increase in Hungary. However, conventional contact tracing of homeless patients is complicated and sometimes even unsuccessful since these individuals are often uncooperative or mobile [92]. In a previous retrospective study using IS6110 RFLP-fingerprinting, we have found a high rate of recent transmission among homeless tuberculosis patients in Budapest similar to other European big cities $[22,88]$. In the present study, we have extended this investigation and revealed the likelihood of a local microepidemy in a district of Budapest with the highest tuberculosis incidence. Traditional epidemiologic methods showed that $16.6 \%$ of these clustered patients were staying in the same homeless shelter at the time of diagnosing the disease. Although, our survey could not confirm any conventional epidemiologic links between the other cases in the cluster it is very likely that these patients have shared the same day time warming places or other non-overnight out-reach units since most of these homeless facilities are in the 8th district of the capital where the patients had lived.

Without the knowledge of genetic diversity of M. tuberculosis in other parts of the country and the prevalence of this strain in non-homeless patients, the historical presence of this clade cannot be completely ruled out since complex ecological, social causes and 
demographical history could also be responsible for the prevalence of some endemic clades throughout long period of times [93, 94]. However, thorough molecular genetic characterization has shown that the observed microepidemy was due to a specific clade that for the time being has not been described elsewhere [66, 67]. The observed spoligotyping patterns also did not exhibit any similarity to spoligotyping patterns of $M$. tuberculosis strains that were recently recovered from $18^{\text {th }}$ century Hungarian mummies in a neighbouring city of Budapest [95]. Although, additional molecular epidemiologic analysis of strains isolated from non-homeless patients in Budapest and in Hungary will also be necessary to determine the actual prevalence of this genotype, since all the patients were newly diagnosed and none of them were elderly, these cases may well represent newly acquired infections due to an actively spreading clade rather than infections or reactivations of a prevalent local lineage. In addition, the high specificity of the ST1773 clade for Budapest is also evident from its distribution within more than 40,000 isolates of the SpolDB4 database from over 130 countries [52, 66, 67]. The MIRU typing, presently among the most accurate complementary techniques to demonstrate the clonality of strains, also confirmed the congruence of all 12 isolates. 


\section{Concluding remarks}

Tuberculosis was recognised as a major global public health problem in the early 1990s, when the World Health Assembly adopted a resolution calling for increased efforts to control the disease. Although, the EU TB notification rates are among the lowest in the in the world, the global TB situation has a recognizable impact on the European situation. To respond to the challenges in TB control, in 2008 ECDC developed the framework for actions to be taken by and within countries, as well as at European Community level, to combat TB. The Action Plan is compatible with both the Global Plan to Stop TB and the plan for highpriority countries in Europe. Within the action plan the strengthening and use of the laboratory services are one of the major points. The laboratories are providing the diagnostic services as well as the molecular typing data important for the monitoring of tuberculosis. As the European efforts cannot be successful without well implemented national planes, ECDC has organised surveillance and laboratory networks to support the Member States.

In every Member State as well as in Hungary, the availability of the prompt and reliable diagnostic service has a crucial importance. In this study several isolation methods were evaluated for Hungarian settings. Our results show that automation of the cultivation process is high on the list of priorities for laboratories dealing with large specimen loads. Although the recently developed MB/BacT (Organon Teknika, Turnhout, Belgium) and ESP II (Difco Laboratories, Detroit, Mich.) culture systems suitable solutions, the capacity of these instruments is rather low (MB/BacT, 240 vials per instrument; ESP II, 384 vials per instrument). Therefore, several units are required, which might be expensive even for laboratories in high-income countries. The automated Bactec MGIT 960 system is a highcapacity, fully automated, continuous-monitoring instrument that can test up to 960 vials for the presence of mycobacteria using non-radiometric fluorescence technology [73, 74].

The recently introduced fully automated Bactec MGIT 960 has been shown to be a viable alternative to the radiometric Bactec 12B, manual Bactec MGIT, ESP II, and $\mathrm{MB} / \mathrm{BacT}$ systems for the rapid and reliable laboratory diagnosis of tuberculosis. In contrast to the Bactec 12B system, it is a non-radiometric assay and there is no need for needles for the inoculation or testing of vials. Further advantages of the fully automated system include no requirement for flushing of vials prior to inoculation, manual loading of racks with vials for each test, and establishment of a reading schedule. Therefore, it is less labour-intensive 
and hence may free laboratory staff for other duties. In addition, the capacity of the automated Bactec MGIT 960 is much higher than that of ESP II or MB/BacT and therefore its application is more useful for laboratories dealing with large numbers of specimens daily. However, in the laboratories with the low specimen load the other low capacity systems might be used. The Hungarian surveillance data show that the laboratory capacity needs strengthening and needs to be better organised. Despite the recently developed methods are available in the country the proportion of the cultured specimens remains below $90 \%$ and the culture confirmation is still not over $50 \%$.

The recent worldwide increase in the incidence of drug-resistant strains of Mycobacterium tuberculosis has highlighted the need for faster and more accurate detection of resistance to RMP, one of the most important anti-tuberculosis drugs. RMP is most effective in killing actively metabolizing $M$. tuberculosis cells, and resistance to RMP often results in high clinical relapse rates. Due to the prolonged turnaround time for conventional susceptibility testing, patients infected with drug-resistant tuberculosis may be inadequately treated and thus remain infectious for longer period than those infected with susceptible strains. Based on collective observations that mutations resulting in an amino acid change within the 81-bp core region of the RNA polymerase-subunit ( $r p o \beta)$ gene are found in more than $96 \%$ of RMP-resistant $M$. tuberculosis strains, several molecular methods have been developed for the rapid (24- to 48-h) detection of mutations in this region. In addition, other studies revealed that mutations associated with RMP resistance can also occur in other regions of the rpo $\beta$ gene, although less frequently. It has also been shown that the information provided by these molecular tests can serve as a molecular epidemiological marker since the relative frequency of the alleles associated with resistance can vary geographically.

Our study demonstrated that frequencies of particular mutations in RMP-resistant M. tuberculosis isolates from East-Hungary are different from those that have been reported for isolates from other geographic areas (Table 4). Our findings demonstrate the importance of validating molecular tests for the detection of RMP resistance using DNA sequence analysis and thus determining the frequencies of particular mutations in the test region before introducing the assay into routine clinical service. However, the use of the line-probe assays for the rapid testing of resistance genes is a useful tool for the screening of MDR-TB even in the low-income countries. The early initiation of the adequate TB treatment may lead to 
better prevention of the transmission of MDR-TB and the more successful control. At this time Hungary belongs to the low incidence countries regarding the MDR-TB. However, the use of the newly developed line-probe assays is recommended to improve the prevention of the transmission of MDR-TB in the country.

In the last decade, several molecular epidemiological methods have been developed in order to obtain adequate information on the $M$. tuberculosis epidemiology and spread, providing the data that traditional epidemiological methods cannot. The molecular epidemiology methods are essential to follow the spread and recent transmission of resistant strains of M. tuberculosis, particularly the MDR/XDR TB strains [96]. Up to recent time, the internationally accepted "gold-standard" method for typing was the IS6110 RFLP-fingerprinting. However, the IS6110 RFLP-fingerprinting is effectively replaced by simpler and faster PCR-based methods, such as the spoligotyping, MIRU and VNTR analysis. The IS6110 transposon element shows a specific distribution and stability within the genome and provided the opportunity to an internationally accepted standardized method to be developed and used. The IS6110 RFLP-fingerprinting method is sensitive enough to differentiate at appropriate level the strains belonging to the same IS6110 genotype family (classification into clusters) from the not related genotypes. The strains classified into a cluster with confirmed epidemiological link reflects the recent transmission among patients.

The Direct Repeat locus of the Mycobacterium tuberculosis complex is a member of the Clustered regularly interspaced short palindromic repeats (CRISPR) sequences family. Spoligotyping is the widely used PCR-based reverse-hybridization blotting technique that assays the genetic diversity of this locus and is useful both for clinical laboratory, molecular epidemiology, evolutionary and population genetics. However, the spoligotyping is less sensitive than the IS6110 RFLP-fingerprinting method, but it perfectly differentiates the dominant genotypes spread around the world. The method is perfectly suitable for genotyping the M. tuberculosis strains with low number of IS6110 bands (e.g. South Asian strains). When used in association with VNTR or MIRU, spoligotyping is a fast, robust, and cost effective genotyping technique, alternative to traditional IS6110 RFLP-fingerprinting. The exact knowledge of the epidemiological situation obtained by molecular epidemiology studies is essential for the successful implementation of the national tuberculosis programs.

The results of this study indicate that more effective control steps are needed to detect and intercept the transmission of drug-resistant tuberculosis in Hungary. The solution would 
be the introduction of real-time cohort analysis of cases by the NTSC in order to increase the adherence of clinicians to national guidelines. In addition, the establishment of reference centres, or teams with expert physicians and microbiologists, for consultation and treatment of drug-resistant cases may also be helpful. Within the EU the laboratory network for molecular surveillance is established and the services are available for all the Member States. More intensive participation of Hungary can lead to better understanding, monitoring and control of MDR-TB at the national level.

The development and the introduction of the new molecular epidemiological methods led to the growing importance of laboratory-based surveillance in addition to the traditional clinical-based surveillance. Using the laboratory-based surveillance the M. tuberculosis strains can be clustered, and thus leads to a deeper understanding of disease epidemiology, as well as the pathways of transmission and the transmission frequency. Quick identification of the contacts and epidemiological links gives an opportunity to take appropriate measures to slow down and finally to stop the spread of infection. The rapid detection of outbreaks and micro-epidemics in closed communities (prisons, elderly homes, day-care settings, schools, homeless shelters, etc.) and in vulnerable populations has the major public health importance.

The present study defined the specific molecular features of a potentially locally emerged M. tuberculosis lineage in Budapest that led to a microepidemy among the homeless in the capital. These data may serve as a reference for better monitoring and understanding of the patterns and transmission dynamics of tuberculosis in this high-risk population and more generally this is the first report on genetic diversity of $M$. tuberculosis in today's Hungarians. The findings also indicate that tuberculosis control and prevention steps among the homeless need to be strengthened in the capital and across the whole country.

Despite the sub-optimal situation in the laboratory surveillance, Hungary has the potential for quick improvements. This study shows that all newly developed diagnostic methods were evaluated in Hungary and those are accessible since 2000. Since 2004, as the member of EU, Hungary has access to all EU funded relevant networks and the lack of own capacity should not have a major impact on the performance. However, improvements in the organisation and use of resources needed as well the more efficient integration of the laboratory services with the national tuberculosis surveillance activities and the national public health programmes. 


\section{Acknowledgements}

The author wishes to express his thanks to:

Professor Erzsébet Nagy, his supervisor, for her excellent support and encouragement;

Dr. Ákos Somoskövi, for introducing him to the field of mycobacteriology; for stimulating discussions, good advices and his outstanding personal support;

Professor Pál Magyar, his supervisor, for his continuous support and encouragement, and for providing the facilities for scientific research;

Professor Imre Hutás, for stimulating discussions and his great personal support;

Dr. Sabine Rüsch-Gerdes, Dr. Stefan Niemann, for sharing their great knowledge and introducing him into the field of research;

Dr. Davide Manissero, for sharing his great knowledge and introducing him into the field of TB monitoring and control;

Zsuzsanna Szilágyi, Katalin Gulyás, for their excellent technical assistance. 


\section{References}

1. Metchock, B.G., F.S. Nolte, and R.J. Wallace, Mycobacterium, in Manual of clinical microbiology, 7th ed., P.R. Murray, et al., Editors. 1999, ASM Press: Washington, D.C. p. 399-437.

2. van Soolingen, D., et al., Molecular epidemiology of tuberculosis in the Netherlands: a nationwide study from 1993 through 1997. J Infect Dis, 1999. 180(3): p. 726-36.

3. Aranaz, A., et al., Mycobacterium tuberculosis subsp. caprae subsp. nov.: a taxonomic study of a new member of the Mycobacterium tuberculosis complex isolated from goats in Spain. Int J Syst Bacteriol, 1999. 49 Pt 3: p. 1263-73.

4. $\quad$ Calmette, A., et al., Premunition des nouveaux-nes contre la tuberculose par le vaccin BCG, 1921-1926. Ann. Inst. Pasteur (Paris), 1926. 40: p. 89-133.

5. $\quad$ Iseman, M.D., What's in a name ... TB or not TB? Tuber Lung Dis, 1996. 77(2): p. 102.

6. Brosch, R., et al., A new evolutionary scenario for the Mycobacterium tuberculosis complex. Proc Natl Acad Sci U S A, 2002. 99(6): p. 3684-9.

7. Schluger, N.W., Recent advances in our understanding of human host responses to tuberculosis. Respir Res, 2001. 2(3): p. 157-63.

8. $\quad$ Schluger, N.W. and W.N. Rom, The host immune response to tuberculosis. Am J Respir Crit Care Med, 1998. 157(3 Pt 1): p. 679-91.

9. Dlugovitzky, D., et al., Circulating profile of Th1 and Th2 cytokines in tuberculosis patients with different degrees of pulmonary involvement. FEMS Immunol Med Microbiol, 1997. 18(3): p. 203-7.

10. Somoskövi, Á., et al., Accessory function and costimulatory molecule expression of alveolar macrophages in patients with pulmonary tuberculosis. Immunobiology, 2000. 201(3-4): p. 450-60.

11. Somoskövi, Á., et al., Different cytokine patterns correlate with the extension of disease in pulmonary tuberculosis. Eur Cytokine Netw, 1999. 10(2): p. 135-42.

12. WHO, Global Tuberculosis Control; a short update to the 2009 report. 2009, World Health Organisation: Geneva.

13. Manangan, L.P. and W.R. Jarvis, Preventing multidrug-resistant tuberculosis and errors in tuberculosis treatment around the globe. Chest, 2000. 117(3): p. 620-3.

14. Somoskövi, Á., L.M. Parsons, and M. Salfinger, The molecular basis of resistance to isoniazid, rifampin, and pyrazinamide in Mycobacterium tuberculosis. Respir Res, 2001. 2(3): p. 164-8.

15. Dye, C. and B.G. Williams, Criteria for the control of drug-resistant tuberculosis. Proc Natl Acad Sci U S A, 2000. 97(14): p. 8180-5.

16. Espinal, M.A., et al., Global trends in resistance to antituberculosis drugs. World Health Organization-International Union against Tuberculosis and Lung Disease Working Group on Anti-Tuberculosis Drug Resistance Surveillance. N Engl J Med, 2001. 344(17): p. 1294-303.

17. Small, P.M. and P.I. Fujiwara, Management of tuberculosis in the United States. N Engl J Med, 2001. 345(3): p. 189-200.

18. ECDC, Tuberculosis surveillance in Europe, 2008. 2010, European Centre for Disease Prevention and Control: Stockholm.

19. WHO, Stop TB Strategy. 2006, Geneva: World Health Organisation. 
20. Hutás, I., [Tuberculosis at the threshold of the 21st century]. Orv Hetil, 1998. 139(19): p. 1139-45.

21. Jónás, J., et al., Annual report of the Hungarian medical care centers in respiratory medicine, 2004. 2005, Korányi National Institute for Tuberculosis and Respiratory Medicine: Budapest, Hungary.

22. Lukács, J., et al., Conventional and molecular epidemiology of tuberculosis in homeless patients in Budapest, Hungary. J Clin Microbiol, 2004. 42(12): p. 5931-4.

23. UN, The Millennium Development Goals Report 2008, New York: United Nations.

24. WHO, Implementing the Stop TB Strategy. A handbook for national tuberculosis control programmes 2008, Geneva: World Health Organisation.

25. WHO/Europe, The Berlin Declaration on Tuberculosis. 2007, Copenhagen: WHO Regional Office for Europe.

26. WHO/Europe, Plan to stop TB in 18 high-priority countries in the European Region, 2007-2015. 2007, Copenhagen: WHO Regional Office for Europe.

27. ECDC, A framework action plan to fight tuberculosis in the European Union. 2008, Stockholm: European Centre for Disease Prevention and Control.

28. Styrt, B.A., et al., Turnaround times for mycobacterial cultures. J Clin Microbiol, 1997. 35(4): p. 1041-2.

29. Tenover, F.C., et al., The resurgence of tuberculosis: is your laboratory ready? J Clin Microbiol, 1993. 31(4): p. 767-70.

30. Gordin, F. and G. Slutkin, The validity of acid-fast smears in the diagnosis of pulmonary tuberculosis. Arch Pathol Lab Med, 1990. 114(10): p. 1025-7.

31. Kim, T.C., et al., Acid-fast bacilli in sputum smears of patients with pulmonary tuberculosis. Prevalence and significance of negative smears pretreatment and positive smears post-treatment. Am Rev Respir Dis, 1984. 129(2): p. 264-8.

32. Salfinger, M. and G.E. Pfyffer, The new diagnostic mycobacteriology laboratory. Eur J Clin Microbiol Infect Dis, 1994. 13(11): p. 961-79.

33. Cohn, M.L., R.F. Waggoner, and J.K. McClatchy, The 7H11 medium for the cultivation of mycobacteria. Am Rev Respir Dis, 1968. 98(2): p. 295-6.

34. Middlebrook, G. and M.L. Cohn, Bacteriology of tuberculosis: laboratory methods. Am J Public Health Nations Health, 1958. 48(7): p. 844-53.

35. McGowan, J.E., Jr., B. Metchock, and F.S. Nolte, Laboratory diagnosis of tuberculosis: past, present, and future. J Med Assoc Ga, 1995. 84(5): p. 215-20.

36. Heifets, L.B., Expedited detection of drug resistance in tuberculosis patients. Ann Emerg Med, 1994. 24(3): p. 457-61.

37. Kent, P.T. and G.P. Kubica, A guide for the level III laboratory. 1985, Atlanta, USA: Centers for Disease Control.

38. Somoskovi, A., et al., [Routine direct detection of Mycobacterium tuberculosis with a rapid test of polymerase chain reaction applied to a Hungarian patient population]. Orv Hetil, 2001. 142(38): p. 2085-90.

39. Somoskövi, Á., et al., Laboratory diagnosis of nontuberculous mycobacteria. Clin Chest Med, 2002. 23(3): p. 585-97.

40. Canetti, G., et al., Advances in techniques of testing mycobacterial drug sensitivity, and the use of sensitivity tests in tuberculosis control programmes. Bull. W. H. O., 1969. 41: p. 21-43. 
41. Hunt, J.M., et al., Detection of a genetic locus encoding resistance to rifampin in mycobacterial cultures and in clinical specimens. Diagn Microbiol Infect Dis, 1994. 18(4): p. 219-27.

42. Heep, M., et al., Mutations in the beginning of the rpoB gene can induce resistance to rifamycins in both Helicobacter pylori and Mycobacterium tuberculosis. Antimicrob Agents Chemother, 2000. 44(4): p. 1075-7.

43. De Beenhouwer, H., et al., Rapid detection of rifampicin resistance in sputum and biopsy specimens from tuberculosis patients by PCR and line probe assay. Tuber Lung Dis, 1995. 76(5): p. 425-30.

44. Jónás, J., et al., Annual report of the Hungarian medical care centers in respiratory medicine, 2003. 2004, Korányi National Institute for Tuberculosis and Respiratory Medicine: Budapest, Hungary.

45. Mester, J., et al., Analysis of tuberculosis surveillance in Hungary in 2000. Int J Tuberc Lung Dis, 2002. 6(11): p. 966-73.

46. Diel, R., et al., Epidemiology of tuberculosis in Hamburg, Germany: long-term population-based analysis applying classical and molecular epidemiological techniques. J Clin Microbiol, 2002. 40(2): p. 532-9.

47. Niemann, S., S. Rusch-Gerdes, and E. Richter, IS6110 fingerprinting of drug-resistant Mycobacterium tuberculosis strains isolated in Germany during 1995. J Clin Microbiol, 1997. 35(12): p. 3015-20.

48. van Embden, J.D., et al., Strain identification of Mycobacterium tuberculosis by DNA fingerprinting: recommendations for a standardized methodology. J Clin Microbiol, 1993. 31(2): p. 406-9.

49. Kremer, K., et al., Comparison of methods based on different molecular epidemiological markers for typing of Mycobacterium tuberculosis complex strains: interlaboratory study of discriminatory power and reproducibility. J Clin Microbiol, 1999. 37(8): p. 2607-18.

50. Pataki, G., Á. Megyesi, and I. Fehér, Annual report of the Hungarian medical care centers in respiratory medicine, 2002. 2003, National Korányi Institute for Tuberculosis and Respiratory Medicine: Budapest.

51. Somoskövi, A., et al., Comparison of recoveries of mycobacterium tuberculosis using the automated BACTEC MGIT 960 system, the BACTEC 460 TB system, and Lowenstein-Jensen medium. J Clin Microbiol, 2000. 38(6): p. 2395-7.

52. Garcia de Viedma, D., et al., Analysis of Mycobacterium tuberculosis genotypes in Madrid and identification of two new families specific to Spain-related settings. J Clin Microbiol, 2005. 43(4): p. 1797-806.

53. Supply, P., et al., Automated high-throughput genotyping for study of global epidemiology of Mycobacterium tuberculosis based on mycobacterial interspersed repetitive units. J Clin Microbiol, 2001. 39(10): p. 3563-71.

54. Sreevatsan, S., et al., Restricted structural gene polymorphism in the Mycobacterium tuberculosis complex indicates evolutionarily recent global dissemination. Proc Natl Acad Sci U S A, 1997. 94(18): p. 9869-74.

55. Rindi, L., et al., Detection of Mycobacterium tuberculosis genotypic groups by a duplex real-time PCR targeting the katG and gyrA genes. J Microbiol Methods, 2004. 59(2): p. 283-7. 
56. Hirano, K., C. Abe, and M. Takahashi, Mutations in the rpoB gene of rifampinresistant Mycobacterium tuberculosis strains isolated mostly in Asian countries and their rapid detection by line probe assay. J Clin Microbiol, 1999. 37(8): p. 2663-6.

57. Matsiota-Bernard, P., G. Vrioni, and E. Marinis, Characterization of rpoB mutations in rifampin-resistant clinical Mycobacterium tuberculosis isolates from Greece. J Clin Microbiol, 1998. 36(1): p. 20-3.

58. Pozzi, G., et al., rpoB mutations in multidrug-resistant strains of Mycobacterium tuberculosis isolated in Italy. J Clin Microbiol, 1999. 37(4): p. 1197-9.

59. Rinder, H., et al., Disequilibria in the distribution of rpoB alleles in rifampicinresistant M. tuberculosis isolates from Germany and Sierra Leone. Microb Drug Resist, 1997. 3(2): p. 195-7.

60. Schilke, K., et al., Universal pattern of RpoB gene mutations among multidrugresistant isolates of Mycobacterium tuberculosis complex from Africa. Int J Tuberc Lung Dis, 1999. 3(7): p. 620-6.

61. Traore, H., et al., Detection of rifampicin resistance in Mycobacterium tuberculosis isolates from diverse countries by a commercial line probe assay as an initial indicator of multidrug resistance. Int J Tuberc Lung Dis, 2000. 4(5): p. 481-4.

62. Valim, A.R., et al., Mutations in the rpoB gene of multidrug-resistant Mycobacterium tuberculosis isolates from Brazil. J Clin Microbiol, 2000. 38(8): p. 3119-22.

63. Williams, D.L., et al., Characterization of rifampin-resistance in pathogenic mycobacteria. Antimicrob Agents Chemother, 1994. 38(10): p. 2380-6.

64. Yuen, L.K., D. Leslie, and P.J. Coloe, Bacteriological and molecular analysis of rifampin-resistant Mycobacterium tuberculosis strains isolated in Australia. J Clin Microbiol, 1999. 37(12): p. 3844-50.

65. Zhang, Y. and A. Telenti, Genetics of drug resistance in Mycobacterium tuberculosis, in Molecular genetics of mycobacteria, G.F. Hatfull and W.R.J. Jacobs, Editors. 2000, ASM Press: Washington, D.C. p. 235-254.

66. Brudey, K., et al., Mycobacterium tuberculosis complex genetic diversity: mining the fourth international spoligotyping database (SpolDB4) for classification, population genetics and epidemiology. BMC Microbiol, 2006. 6: p. 23.

67. Filliol, I., et al., Snapshot of moving and expanding clones of Mycobacterium tuberculosis and their global distribution assessed by spoligotyping in an international study. J Clin Microbiol, 2003. 41(5): p. 1963-70.

68. Filliol, I., et al., Global phylogeny of Mycobacterium tuberculosis based on single nucleotide polymorphism (SNP) analysis: insights into tuberculosis evolution, phylogenetic accuracy of other DNA fingerprinting systems, and recommendations for a minimal standard SNP set. J Bacteriol, 2006. 188(2): p. 759-72.

69. Gutacker, M.M., et al., Single-nucleotide polymorphism-based population genetic analysis of Mycobacterium tuberculosis strains from 4 geographic sites. J Infect Dis, 2006. 193(1): p. 121-8.

70. Pfyffer, G.E., et al., Comparison of the Mycobacteria Growth Indicator Tube (MGIT) with radiometric and solid culture for recovery of acid-fast bacilli. J Clin Microbiol, 1997. 35(2): p. 364-8.

71. Somoskövi, Á. and P. Magyar, Comparison of the mycobacteria growth indicator tube with MB redox, Lowenstein-Jensen, and Middlebrook 7 H11 media for recovery of mycobacteria in clinical specimens. J Clin Microbiol, 1999. 37(5): p. 1366-9. 
72. Piersimoni, C., et al., Multicenter evaluation of the MB-Redox medium compared with radiometric BACTEC system, mycobacteria growth indicator tube (MGIT), and Lowenstein-Jensen medium for detection and recovery of acid-fast bacilli. Diagn Microbiol Infect Dis, 1999. 34(4): p. 293-9.

73. Hanna, B.A., et al., Multicenter evaluation of the BACTEC MGIT 960 system for recovery of mycobacteria. J Clin Microbiol, 1999. 37(3): p. 748-52.

74. Tortoli, E., et al., Use of BACTEC MGIT 960 for recovery of mycobacteria from clinical specimens: multicenter study. J Clin Microbiol, 1999. 37(11): p. 3578-82.

75. Tortoli, E., et al., Multicenter comparison of ESP Culture System II with BACTEC $460 T B$ and with Lowenstein-Jensen medium for recovery of mycobacteria from different clinical specimens, including blood. J Clin Microbiol, 1998. 36(5): p. 137881.

76. Woods, G.L., et al., Clinical evaluation of difco ESP culture system II for growth and detection of mycobacteria. J Clin Microbiol, 1997. 35(1): p. 121-4.

77. Benjamin, W.H., Jr., et al., Comparison of the MB/BacT system with a revised antibiotic supplement kit to the BACTEC 460 system for detection of mycobacteria in clinical specimens. J Clin Microbiol, 1998. 36(11): p. 3234-8.

78. Cooksey, R.C., et al., Evaluation of the invader assay, a linear signal amplification method, for identification of mutations associated with resistance to rifampin and isoniazid in Mycobacterium tuberculosis. Antimicrob Agents Chemother, 2000. 44(5): p. 1296-301.

79. Goble, M., et al., Treatment of 171 patients with pulmonary tuberculosis resistant to isoniazid and rifampin. N Engl J Med, 1993. 328(8): p. 527-32.

80. Heep, M., et al., Frequency of rpoB mutations inside and outside the cluster I region in rifampin-resistant clinical Mycobacterium tuberculosis isolates. J Clin Microbiol, 2001. 39(1): p. 107-10.

81. Rossau, R., et al., Evaluation of the INNO-LiPA Rif. TB assay, a reverse hybridization assay for the simultaneous detection of Mycobacterium tuberculosis complex and its resistance to rifampin. Antimicrob Agents Chemother, 1997. 41(10): p. 2093-8.

82. Taniguchi, H., et al., Rifampicin resistance and mutation of the rpoB gene in Mycobacterium tuberculosis. FEMS Microbiol Lett, 1996. 144(1): p. 103-8.

83. Hermans, P.W., et al., Analysis of the population structure of Mycobacterium tuberculosis in Ethiopia, Tunisia, and The Netherlands: usefulness of DNA typing for global tuberculosis epidemiology. J Infect Dis, 1995. 171(6): p. 1504-13.

84. Krüüner, A., et al., Spread of drug-resistant pulmonary tuberculosis in Estonia. J Clin Microbiol, 2001. 39(9): p. 3339-45.

85. Sajduda, A., et al., Molecular epidemiology of drug-resistant Mycobacterium tuberculosis strains isolated from patients with pulmonary tuberculosis in Poland: $a$ 1-year study. Int J Tuberc Lung Dis, 2004. 8(12): p. 1448-57.

86. Kubica, T., S. Rusch-Gerdes, and S. Niemann, The Beijing genotype is emerging among multidrug-resistant Mycobacterium tuberculosis strains from Germany. Int J Tuberc Lung Dis, 2004. 8(9): p. 1107-13.

87. Vukovic, D., et al., Molecular epidemiology of pulmonary tuberculosis in Belgrade, central serbia. J Clin Microbiol, 2003. 41(9): p. 4372-7.

88. Gutierrez, M.C., et al., Molecular fingerprinting of Mycobacterium tuberculosis and risk factors for tuberculosis transmission in Paris, France, and surrounding area. J Clin Microbiol, 1998. 36(2): p. 486-92. 
89. Flament-Saillour, M., et al., Outcome of multi-drug-resistant tuberculosis in France: a nationwide case-control study. Am J Respir Crit Care Med, 1999. 160(2): p. 587-93.

90. Glynn, J.R., et al., Worldwide occurrence of Beijing/W strains of Mycobacterium tuberculosis: a systematic review. Emerg Infect Dis, 2002. 8(8): p. 843-9.

91. Toungoussova, O.S., et al., Spread of drug-resistant Mycobacterium tuberculosis strains of the Beijing genotype in the Archangel Oblast, Russia. J Clin Microbiol, 2002. 40(6): p. 1930-7.

92. Li, J., et al., Finding contacts of homeless tuberculosis patients in New York City. Int J Tuberc Lung Dis, 2003. 7(12 Suppl 3): p. S397-404.

93. Day, N.P., et al., A link between virulence and ecological abundance in natural populations of Staphylococcus aureus. Science, 2001. 292(5514): p. 114-6.

94. McGrath, J.W., Social networks of disease spread in the lower Illinois valley: a simulation approach. Am J Phys Anthropol, 1988. 77(4): p. 483-96.

95. Fletcher, H.A., et al., Molecular analysis of Mycobacterium tuberculosis DNA from a family of 18th century Hungarians. Microbiology, 2003. 149(Pt 1): p. 143-51.

96. Mostrom, P., et al., Methods used in the molecular epidemiology of tuberculosis. Clin Microbiol Infect, 2002. 8(11): p. 694-704. 
Annex 1. The list of the publications on which the Thesis are based 


\title{
Comparison of Recoveries of Mycobacterium tuberculosis Using the Automated BACTEC MGIT 960 System, the BACTEC 460 TB System, and Löwenstein-Jensen Medium
}

\author{
ÁKOS SOMOSKÖVI,* CSABA KÖDMÖN, ÁKOS LANTOS, ZOLTÁN BÁRTFAI, \\ LILLA TAMÁSI, JUDIT FÜZY, AND PÁL MAGYAR \\ Department of Respiratory Medicine, Semmelweis University, H-1536 Budapest 114, Hungary
}

Received 12 October 1999/Returned for modification 29 January 2000/Accepted 16 February 2000

\begin{abstract}
Using two different liquid media and one conventional solid medium, a total of $\mathbf{5 7}$ mycobacterial isolates (Mycobacterium tuberculosis, $n=55$; nontuberculous mycobacteria, $n=2$ ) were recovered from 377 clinical specimens. The rates of recovery of $M$. tuberculosis were $96.4 \%$ with the BACTEC MGIT 960 liquid medium, 92.7\% with BACTEC 12B liquid medium, and $81.8 \%$ with the Löwenstein-Jensen (LJ) medium. The mean time to detection of $M$. tuberculosis in smear-positive specimens was 12.6 days for BACTEC MGIT 960 medium, 13.8 days for BACTEC 12B medium, and 20.1 days for $\mathrm{LJ}$ medium, and in smear-negative specimens it was 15.8 days for BACTEC MGIT 960 medium, 17.7 days for BACTEC 12B medium, and 42.2 days for LJ medium. The rates of contamination were 3.7, 2.9, and $1.2 \%$ for the BACTEC MGIT 960, BACTEC 12B, and LJ media, respectively. In conclusion, the nonradiometric, fully automated 7-mI BACTEC MGIT 960 system can be considered a viable alternative to the semiautomated, radiometric BACTEC 460 TB system.
\end{abstract}

The use of the radiometric BACTEC 460 TB broth-based system (Becton Dickinson Diagnostic Instrument Systems, Sparks, Md.) considerably improves the recovery of and decreases the time required to detect mycobacteria; however, this procedure is still labor-intensive and requires attention to special safety and regulatory issues regarding radioisotopes (7). Previous reports have demonstrated that the 4-ml Mycobacteria Growth Indicator Tube (MGIT; BBL Becton Dickinson Microbiology Systems, Cockeysville, Md.) and MB Redox (Biotest AG, Dreieich, Germany) systems are suitable nonradiometric alternatives to BACTEC $460 \mathrm{~TB}(4,5,8)$. However, these methods still require manual processing and are best suited for laboratories which cannot afford or, due to the low number of processed specimens, do not need instrumentation. Automation of the cultivation process is high on the list of priorities for laboratories dealing with large specimen loads. Although the recently developed MB/BacT (Organon Teknika, Turnhout, Belgium) and ESP II (Difco Laboratories, Detroit, Mich.) culture systems provide a fully automated, walk-away cultivation process, the capacity of these instruments is rather low (MB/BacT, 240 vials per instrument; ESP II, 384 vials per instrument) $(1,6,9,11)$. Therefore, several units are required, which might be expensive even for laboratories in high-income countries. The BACTEC MGIT 960 system is a high-capacity, fully automated, continuous-monitoring instrument that can test up to 960 7-ml MGIT vials for the presence of mycobacteria using nonradiometric fluorescence technology $(2,10)$. The culture vials contain a fluorescent sensor that responds to the concentration of oxygen in the culture medium. The instrument's photodetectors measure the fluorescence in each vial every $60 \mathrm{~min}$. The level of fluorescence corresponds to the amount of oxygen consumed by the organisms in the inoculated specimens, and this, in turn, is proportional to the number of bacteria present. When a certain level of fluorescence is

\footnotetext{
* Corresponding author. Mailing address: Department of Respiratory Medicine, Laboratory for Mycobacteria, Semmelweis Medical School, P.O. Box 250, H-1536 Budapest 114, Hungary. Phone: 36-1355-8682. Fax: 36-1-214-2498. E-mail: akos@pulm.sote.hu.
}

reached, the instrument indicates that the vial is positive. The purpose of this study was to evaluate the fully automated 7-ml BACTEC MGIT 960 system for the detection of mycobacteria in clinical specimens and compare the results with those of the reference BACTEC 460 TB system and of Löwenstein-Jensen (LJ) solid medium in terms of recovery rate, mean time to detection, and contamination rate.

The Department of Respiratory Medicine of Semmelweis Medical School is a university-based tertiary care medical center with its own laboratory facility. A total of 377 consecutive clinical specimens (288 sputum, 51 bronchoalveolar lavage or bronchial mucus aspirate, 32 gastric juice, and 6 pleural effusion) from 243 patients were processed between 29 March 1999 and 31 May 1999. All patients were human immunodeficiency virus negative. All clinical specimens were digested and decontaminated by the $\mathrm{N}$-acetyl-L-cysteine- $\mathrm{NaOH}$ method as described by Kent and Kubica (3). A 4\% concentration (starting concentration) of $\mathrm{NaOH}$ was used. After decontamination, smears were prepared from the concentrated sediments of the specimens for Ziehl-Neelsen (ZN) acid-fast staining. The remaining sediment was suspended in $1.5 \mathrm{ml}$ of sterile phosphate-buffered saline ( $\mathrm{pH}$ 6.8). Before inoculation, BACTEC MGIT 960 and BACTEC 12B vials were supplemented as described by the manufacturer. We inoculated 0.5 $\mathrm{ml}$ of the processed specimen into BACTEC MGIT 960, $0.5 \mathrm{ml}$ into BACTEC $12 \mathrm{~B}$, and $0.2 \mathrm{ml}$ onto each of two LJ medium slants. All inoculated media were incubated at $37^{\circ} \mathrm{C}$. BACTEC MGIT 960 vials were introduced into the BACTEC MGIT 960 instrument as recommended by the manufacturer and tested either until they were found to be positive or for 6 weeks. The BACTEC 12B vials were read twice weekly for the first 2 weeks and weekly thereafter for 4 weeks. When the growth index of a BACTEC $12 \mathrm{~B}$ vial reached $\geq 10$, the vial was tested daily until the vial attained a growth index of $\geq 100$, at which time it was considered presumptively positive. If no ${ }^{14} \mathrm{CO}_{2}$ production was detected after 6 weeks, the BACTEC 12B vial was regarded as negative. LJ medium slants were examined weekly for 8 weeks for the visible appearance of colonies. After confirmation of mycobacterial growth in a liquid or solid medium, 
TABLE 1. Rates of recovery of mycobacteria and contaminants by BACTEC MGIT 960, BACTEC 12B, and LJ medium

\begin{tabular}{llllr}
\hline \multirow{2}{*}{ Medium } & \multicolumn{3}{c}{ No. $(\%)$ of isolates recovered ${ }^{a}$} \\
\cline { 2 - 5 } & $\begin{array}{c}\text { All } \\
\text { organisms }\end{array}$ & M. tuberculosis & NTM & Contaminants \\
\hline BACTEC MGIT 960 & $55(96.5)$ & $53(96.4)$ & $2(100)$ & $14(3.7)$ \\
BACTEC 12B & $53(93.0)$ & $51(92.7)$ & $2(100)$ & $11(2.9)$ \\
LJ & $46(80.7)$ & $45(81.8)$ & $1(50)$ & $4(1.2)$ \\
\hline
\end{tabular}

${ }^{a}$ The total number of organisms recovered was 57 , of which 55 were $M$. tuberculosis, 2 were NTM, and 17 were contaminants. $\chi^{2}$ test for differences in recovery of mycobacteria: BACTEC MGIT 960 versus LJ medium, $P<0.05$ (significant). $\chi^{2}$ test for differences in recovery of $M$. tuberculosis: BACTEC MGIT 960 versus LJ medium, $P<0.05$ (significant).

the parallel media were read daily. On the day of detection, all positive liquid and solid media were examined by $\mathrm{ZN}$ staining to confirm the presence of acid-fast bacteria (AFB) and subcultured onto Columbia agar with $5 \%$ sheep blood (bioMerieux Microbiology Systems, Marcy l'Etoile, France) to check for contaminants. Cultures found AFB positive by microscopy were identified by means of the AccuProbe culture identification test (Gen-Probe, San Diego, Calif.) and conventional biochemical tests (3). The $\chi^{2}$ test was used to evaluate differences between recovery rates in different media. Analysis of variance (ANOVA) and the Newman-Keuls test were used to establish significant differences in relation to the duration of growth.

A total of 57 specimens $(15.1 \%)$ were positive for mycobacteria, of which $14(24.6 \%)$ were AFB smear positive and 43 (75.4\%) were AFB smear negative. The mycobacterial species identified were Mycobacterium tuberculosis $(n=55)$, Mycobacterium avium complex $(n=1)$, and Mycobacterium xenopi $(n=$ 1 ). The numbers of isolates of mycobacteria recovered by BACTEC MGIT 960, BACTEC 12B, and LJ medium are presented in Table 1. As a single medium, BACTEC MGIT 960 recovered $53(96.4 \%)$ of the $55 \mathrm{M}$. tuberculosis isolates, BACTEC 12B recovered $51(92.7 \%)$ of the 55 isolates, and LJ medium recovered $45(81.8 \%)$ of the 55 isolates. A statistically significant difference was demonstrated between BACTEC MGIT 960 and LJ medium $(P<0.05)$.

In the present study, the automated 7-ml BACTEC MGIT 960 system displayed a rate of recovery $(96.4 \%)$ of $M$. tuberculosis higher than those previously reported for the manual 4-ml MGIT, i.e., $89.4 \%$ by Pfyffer et al. (4), $81.3 \%$ by Somoskövi and Magyar (8), and $85 \%$ by Piersimoni et al. (5), and also higher than those previously reported for the same walk-away system, i.e., $77 \%$ by Hanna et al. (2) and $88 \%$ by Tortoli et al. (10). The 7-ml BACTEC MGIT 960 was also more efficient than the similarly fully automated ESP II system (sensitivity, 85.3 and $89 \%)(9,11)$ and showed a sensitivity comparable to that of the MB/BacT system (96\%) (1).

The lower rate of recovery observed by Hanna et al. and Tortoli et al. may have been due to a higher contamination rate with the 7-ml BACTEC MGIT 960. Hanna et al. found that after removal of the contaminated cohorts from their analysis, the sensitivity of BACTEC MGIT 960 increased from 77 to $86 \%$ (2). Contamination did not cause a serious problem in our study. The rates of contamination were $3.7,2.9$, and $1.2 \%$ for BACTEC MGIT 960, BACTEC 12B, and LJ medium, respectively (Table 1). We found that the rate of contamination with the 7-ml Bactec MGIT 960 was lower than the values reported previously for other walk-away broth-based systems $(1,2,9,10$, 11) but was in line with the rates of contamination observed with the 4-ml MGIT $(4,5,8)$. However, the concentration of
$\mathrm{NaOH}$ used in the other walk-away system studies was lower $(2 \%)$ than that used both in the present study (4\%) and in the 4-ml MGIT studies (3\%) (1, 2, 4, 5, 8, 9, 10, 11). Moreover, the delay between specimen collection and processing due to longer specimen shipment times at some sites (i.e., 2 to 5 days at one of the test sites in the study by Hanna et al.) and the different patient population may also explain these discrepancies $(2,4)$. Our laboratory usually receives the majority of samples within $30 \mathrm{~min}$ after collection. This rapid delivery could impact both the contamination rate and the rate of recovery.

Two M. tuberculosis isolates grew in the 7-ml BACTEC MGIT 960 but not on BACTEC 12B or LJ medium. BACTEC $12 \mathrm{~B}$ or $\mathrm{LJ}$ medium did not detect any isolates alone. It is possible that those two isolates recovered by the 7-ml MGIT alone did not metabolize the $\left[{ }^{14} \mathrm{C}\right]$ palmitic acid in BACTEC $12 \mathrm{~B}$ or that the higher volume of the 7-ml BACTEC MGIT 960 diluted potential growth inhibitors in the specimen. Each system detected all 14 smear-positive specimens, and all of these contained M. tuberculosis. For the smear-negative specimens, the M. tuberculosis recovery rates were 39 (95.1\%) of 41 isolates with BACTEC MGIT 960, $37(90.2 \%)$ of 41 isolates with BACTEC 12B, and $31(75.6 \%)$ of 41 isolates with LJ medium. Again, a statistically significant difference was found between BACTEC MGIT 960 and LJ medium $(P<0.05)$. The number of nontuberculous mycobacteria (NTM) in this study was too low to allow a meaningful statistical comparison for this group.

It is generally recommended that a solid medium not be used alone but be used in combination with a liquid-based culture system to increase the sensitivity of cultivation for mycobacteria $(3,4,7)$. This combination is considered to be the "gold standard." Recovery rates were also compared when each liquid medium was combined with LJ medium. The recovery rates obtained for $M$. tuberculosis were $53(96.4 \%)$ of 55 isolates with BACTEC MGIT 960 plus LJ medium and 51 $(92.7 \%)$ of 55 isolates with BACTEC $12 \mathrm{~B}$ plus LJ medium. The statistical analysis did not reveal any significant difference between the two combinations. Therefore, combination of the 7-ml BACTEC MGIT 960 and LJ medium could be a reliable alternative for the standard liquid radiometric plus solid medium combination. The rate of recovery of $M$. tuberculosis found in our study for the 7-ml BACTEC MGIT 960 plus solid medium was in line with the findings of $92 \%$ reported by Pfyffer et al. (4), $94.6 \%$ reported by Somoskövi and Magyar (8), and $92 \%$ reported by Piersimoni et al. (4) for the 4-ml MGIT plus solid medium; $94 \%$ reported by Woods et al. (11) and $96.1 \%$ reported by Tortoli et al. (9) for ESP II plus solid medium; and $97 \%$ reported by Hanna et al. (2) and $94 \%$ reported by Tortoli et al. (10) for the 7-ml Bactec MGIT 960 plus solid medium.

The mean (range) times to detection of all M. tuberculosis isolates were 14.3 (6 to 24 ), 16.6 (8 to 23 ), and 35.8 (14 to 58) days with BACTEC MGIT 960, BACTEC 12B, and LJ medium, respectively. ANOVA and the Newman-Keuls test revealed statistically significant differences between BACTEC MGIT 960 and LJ medium and between BACTEC 12B and LJ medium $(P<0.001$ and $P<0.001$, respectively). The difference between the two liquid media was not statistically significant. The mean times to detection of growth of all mycobacteria and $M$. tuberculosis with regard to results of acid-fast microscopy are listed in Table 2 . The times to detection of $M$. tuberculosis from smear-positive specimens were comparable for the 7-ml BACTEC MGIT 960 and BACTEC 12B (12.6 versus 13.8 days). The time to detection with the 7-ml BACTEC MGIT 960 was shorter than the time of 15.3 days reported by Piersimoni et al. (5) for the manual MGIT but was 
TABLE 2. Mean time to detection of all mycobacteria and M. tuberculosis in clinical specimens

\begin{tabular}{lllc}
\hline \multirow{2}{*}{\multicolumn{1}{c}{ Medium }} & \multicolumn{3}{c}{ Mean no. of days (range) to detection ${ }^{a}$ of: } \\
\cline { 2 - 4 } & & \multicolumn{2}{c}{ M. tuberculosis } \\
\cline { 2 - 3 } & Mycobacteria & $\begin{array}{c}\text { Smear } \\
\text { positive }\end{array}$ & $\begin{array}{c}\text { Smear } \\
\text { negative }\end{array}$ \\
\hline BACTEC MGIT 960 & $13.2(6-24)$ & $12.6(8-18)$ & $15.8(6-24)$ \\
BACTEC 12B & $16.8(8-23)$ & $13.8(8-23)$ & $17.7(9-23)$ \\
LJ & $36.2(14-58)$ & $20.1(14-27)$ & $42.2(18-58)$ \\
\hline
\end{tabular}

${ }^{a}$ ANOVA, $P<0.001$. Newman-Keuls test for differences in mean times to detection of mycobacteria and M. tuberculosis: BACTEC MGIT 960 versus LJ medium, $P<0.001$ (significant), BACTEC 12B versus LJ medium, $P<0.001$ (significant).

longer than the time of 9.9 days observed by Pfyffer et al. (4) and the 7.2 days reported by Somoskövi and Magyar (8), both for the 4-ml MGIT, and the 10.6 days reported by Hanna et al. (2) and the 12.5 days reported by Tortoli et al. (10) for the 7-ml BACTEC MGIT 960. In comparison with other walk-away systems, the time to detection of M. tuberculosis with the 7-ml BACTEC MGIT 960 in this study was lower than the 14.5 days found by Woods et al. (11) for ESP II but was higher than the 10.3 days given by Benjamin et al. (1) for MB/BacT. However, the proportion of smear-positive specimens was not exactly the same in all of the studies.

In our study, the mean time to detection of M. tuberculosis in smear-negative specimens was slightly shorter with the 7-ml BACTEC MGIT 960 than with BACTEC 12B (15.8 versus 17.2 days). Also, the time to detection with the 7-ml BACTEC MGIT 960 was shorter than those reported by Hanna et al. (18.1 days) (2), Tortoli et al. (19.6 days) (10), Pfyffer et al. (20.3 days) (4), Somoskövi and Magyar (19.1 days) (8), and Piersimoni et al. (22.4 days) (5) for either the 7-ml BACTEC MGIT 960 or the manual 4-ml MGIT. The mean time to detection of M. tuberculosis in smear-negative specimens with the 7-ml BACTEC MGIT 960 was also much shorter than those reported for ESP II and MB/BacT, i.e., 18.9 days by Woods et al. (11) and 20.1 days by Benjamin et al. (1). Our results indicate that the automated 7-ml BACTEC MGIT 960 system may be much faster for the recovery of $M$. tuberculosis from smearnegative specimens than the manual 4-ml MGIT method and the similarly automated broth-based systems.

We did not observe any false-positive cultures (instrument positive but smear and Columbia agar subculture negative) with BACTEC MGIT 960 during the study. However, in eight BACTEC MGIT 960 vials signaled to be positive by the instrument, the confirmatory acid-fast microscopy was negative and the presence of AFB could be detected on $\mathrm{ZN}$-stained smears only after incubation for 3 to 4 more days.

In summary, the recently introduced fully automated 7-ml Bactec MGIT 960 has been shown to be a viable alternative to the radiometric BACTEC 460 TB, manual 4-ml MGIT, ESP II, and $\mathrm{MB} / \mathrm{BacT}$ systems for the rapid and reliable laboratory diagnosis of tuberculosis. In contrast to the BACTEC $460 \mathrm{~TB}$ system, it is a nonradiometric assay and there is no need for needles for the inoculation or testing of vials. Further advantages of the fully automated system include no requirement for flushing of vials prior to inoculation, manual loading of racks with vials for each test, and establishment of a reading schedule. Therefore, it is less labor-intensive and hence may free laboratory staff for other duties. In addition, the capacity of the 7-ml BACTEC MGIT 960 is much higher than that of ESP II or $\mathrm{MB} / \mathrm{BacT}$ and therefore its application is more useful for laboratories dealing with large numbers of specimens daily.

We thank L. M. Parsons, M. Salfinger, and M. Magyar for their stimulating discussions and critical review of the manuscript and Z. S. Szilágyi, K. Gulyás, and Á. Dombai for their excellent technical assistance.

A. Somoskövi was supported in part by grant 1D43TW00915 from the Fogarty International Center, National Institutes of Health.

\section{REFERENCES}

1. Benjamin, W. H., Jr., K. B. Waites, A. Beverly, L. Gibbs, M. Waller, S. Nix, S. A. Moser, and M. Willert. 1998. Comparison of the MB/BacT system with a revised antibiotic supplement kit to the Bactec 460 system for detection of mycobacteria in clinical specimens. J. Clin. Microbiol. 36:3234-3238.

2. Hanna, B. A., A. Ebrahimzadeh, B. Elliot, M. A. Morgan, S. M. Novak, S. Rüsch-Gerdes, M. Acio, D. F. Dunbar, T. M. Holmes, C. H. Rexer, C. Savthyakumar, and A. M. Vannier. 1999. Multicenter evaluation of the Bactec MGIT 960 system for recovery of mycobacteria. J. Clin. Microbiol. 37:748-752

3. Kent, P. T., and G. P. Kubica. 1985. Public health mycobacteriology. A guide for a level III laboratory. Centers for Disease Control, Atlanta, Ga

4. Pfyffer, G. E., H.-M. Welscher, P. Kissling, C. Cieslak, M. J. Casal, J. Gutierrez, and S. Rüsch-Gerdes. 1997. Comparison of the Mycobacteria Growth Indicator Tube (MGIT) with radiometric and solid culture for recovery of acid-fast bacilli. J. Clin. Microbiol. 35:364-368.

5. Piersimoni, C., C. Scarparo, P. Cichero, M. Del Pezzo, I. Covelli, G. Gesu, D. Nista, M. Scagnelli, and F. Mandler. 1999. Multicenter evaluation of the MB-Redox medium compared with radiometric Bactec system, Mycobacteria Growth Indicator Tube (MGIT), and Löwenstein-Jensen medium for detection and recovery of acid-fast bacilli. Diagn. Microbiol. Infect. Dis. 34:293-299.

6. Rohner, P., B. Ninet, C. Metral, S. Emler, and R. Auckenthaler. 1997. Evaluation of the $\mathrm{MB} / \mathrm{BacT}$ system and comparison to the Bactec 460 system and solid media for isolation of mycobacteria from clinical specimens. J. Clin. Microbiol. 35:3127-3131.

7. Salfinger, M., and G. E. Pfyffer. 1994. The new diagnostic mycobacteriology laboratory. Eur. J. Clin. Microbiol. Infect. Dis. 11:961-979.

8. Somoskövi, Á., and P. Magyar. 1999. Comparison of the Mycobacteria Growth Indicator Tube with MB Redox, Löwenstein-Jensen, and Middlebrook 7H11 media for recovery of mycobacteria in clinical specimens. J. Clin. Microbiol. 37:1366-1369.

9. Tortoli, E., P. Chichero, M. G. Chirillo, M. R. Gismondo, L. Bono, G. Gesu, M. T. Simonetti, G. Volpe, G. Nardi, and P. Marone. 1998. Multicenter comparison of ESP culture system II with Bactec 460 TB and with Löwenstein-Jensen medium for recovery of mycobacteria from different clinical specimens, including blood. J. Clin. Microbiol. 36:1378-1381.

10. Tortoli, E., P. Cichero, C. Piersimoni, M. T. Simonetti, G. Gesu, and D. Nista. 1999. Use of Bactec MGIT 960 for recovery of mycobacteria from clinical specimens: multicenter study. J. Clin. Microbiol. 37:3578-3582.

11. Woods, G. L., G. Fish, M. Plaunt, and T. Murphy. 1997. Clinical evaluation of Difco ESP culture system II for growth and detection of mycobacteria. J. Clin. Microbiol. 35:121-124. 
II. 


\title{
Molecular Characterization of Rifampin-Resistant Isolates of Mycobacterium tuberculosis from Hungary by DNA Sequencing and the Line Probe Assay
}

\author{
ZOLTÁN BÁRTFAI, ${ }^{1}$ ÁKOS SOMOSKÖVI, ${ }^{1,2 *}$ CSABA KÖDMÖN, ${ }^{1}$ NÓRA SZABÓ, $, 3,4$ \\ ERZSÉBET PUSKÁS, ${ }^{5}$ LÁSZLÓNÉ KOSZTOLÁNYI, ${ }^{5}$ ESZTER FARAGÓ, ${ }^{6}$ \\ JUDIT MESTER, ${ }^{4}$ LINDA M. PARSONS, ${ }^{2}$ AND MAX SALFINGER ${ }^{2,7,8}$
}

Department of Respiratory Medicine, School of Medicine, Semmelweis University, ${ }^{1}$ and Korányi National Institute for Tuberculosis and Respiratory Medicine, ${ }^{4}$ Budapest, Prodia Laboratory for Mycobacteria, Jósa Hospital, Nyíregyháza, ${ }^{3}$ Borsod-Abaúj-Zeplén County Bureau of Public Health and Medical Officers, Miskolc, ${ }^{5}$ and Laboratory for Mycobacteria, School of Medicine, University of Debrecen, Debrecen, ${ }^{6}$ Hungary, and Wadsworth Center, New York State Department of Health, ${ }^{2}$ Department of Medicine, Albany Medical College, ${ }^{7}$ and Department of Biomedical Sciences, School of Public Health,

State University of New York at Albany, ${ }^{8}$ Albany, New York

Received 24 January 2001/Returned for modification 2 May 2001/Accepted 30 May 2001

\begin{abstract}
Two regions of $r p o B$ associated with rifampin resistance were sequenced in 29 rifampin-resistant (determined by the proportion method) isolates of Mycobacterium tuberculosis obtained from patients from three counties in Hungary. Of the 29 resistant strains, 27 had a mutation in either the 81-bp region (26 strains) or the $\mathrm{N}$-terminal region ( 1 strain), while the other 2 strains had no mutations in either region. The locations and frequencies of the mutations differed from those previously reported. The most common mutation in this study, D516V, was found in 38\% of the Hungarian strains, a frequency 2 to 10 times higher than that found in studies from other countries. These same 29 isolates were also evaluated with the Inno-LiPA Rif. TB test (LiPA), a reverse hybridization assay for the rapid detection of rifampin resistance. Although LiPA detected the presence of an $r p o B$ mutation in 26 of the resistant isolates, the type of mutation could not be determined in 4 isolates because the mutations present were not among those included on the LiPA strip. In addition, a silent mutation in one of the rifampin-susceptible control strains was interpreted as rifampin resistant by LiPA. These findings demonstrate the importance of validating this rapid molecular test by comparison with DNA sequence results in each geographic location before incorporating the test into routine diagnostic work.
\end{abstract}

The recent worldwide increase in the incidence of drugresistant strains of Mycobacterium tuberculosis has highlighted the need for faster and more accurate detection of resistance to rifampin (RMP), one of the most important antituberculosis drugs (17). RMP is most effective in killing actively metabolizing M.tuberculosis, and resistance to RMP often results in high clinical relapse rates $(5,15)$. Because of the prolonged turnaround time for conventional susceptibility testing, patients infected with drug-resistant tuberculosis may be inadequately treated and thus remain infectious for longer periods than those infected with susceptible strains.

Based on collective observations that mutations resulting in an amino acid change within the 81-bp core region of the RNA polymerase $\beta$-subunit $(r p o B)$ gene are found in more than $96 \%$ of RMP-resistant M. tuberculosis strains, several molecular methods have been developed for the rapid (24- to 48-h) detection of mutations in this region $(3,10,16,18,24,25,28-30)$. In addition, other studies revealed that mutations associated with RMP resistance can also occur in other regions of the $r p o B$ gene, although less frequently $(6,7,23)$. It has also been shown elsewhere that the information provided by these molecular tests can serve as a molecular epidemiological marker

\footnotetext{
* Corresponding author. Mailing address: Wadsworth Center, New York State Department of Health, P.O. Box 509, Albany, NY 122010509. Phone: (518) 474-2196. Fax: (518) 474-6964. E-mail: somoskov $@$ wadsworth.org or medve@pulm.sote.hu.
}

since the relative frequency of the alleles associated with resistance can vary geographically $(10,20)$.

Therefore, the aim of the present study was to determine the drug resistance profile of 29 RMP-resistant M. tuberculosis isolates obtained in East Hungary and to detect and identify mutations present in the $r p o B$ gene. Two molecular assays were used. In the first, two regions of $r p o B$ that have been associated with RMP resistance were amplified by PCR and the DNA sequence was determined. The results of the DNA sequencing were then compared with results from a commercially available rapid test, the PCR-based reverse hybridization line probe assay (Inno-LiPA Rif. TB Test [LiPA]; Innogenetics N.V., Ghent, Belgium).

After 20 years of decline, the incidence of pulmonary tuberculosis in Hungary increased by $18.1 \%$ between 1990 and 1999 (rising from 31.0 to 36.6 per 100,000 inhabitants) (1). In addition, East Hungary (Borsod-Abaúj-Zemplén, Hajdú-Bihar, and Szabolcs-Szatmár-Bereg counties) is the part of the country with the highest incidence of tuberculosis generally (38.7, 51.5, and 56.7 per 100,000 inhabitants, respectively) and drugresistant tuberculosis specifically (1). In 1999, these three counties collectively reported 888 of the 3,912 (22.7\%) tuberculosis cases in Hungary. The 29 RMP-resistant isolates examined in this study were isolated from patients in these three counties during 1999 (1).

The M. tuberculosis H37Rv ATCC 27294 strain and six clin- 
TABLE 1. Resistance patterns of RMP-resistant M. tuberculosis isolates from Hungary by the proportion method, the line probe assay, and DNA sequencing of the 81-bp region of the $r p o B$ gene $^{a}$

\begin{tabular}{|c|c|c|c|c|c|c|c|c|c|c|c|c|c|c|c|c|}
\hline \multirow{3}{*}{ Strain } & \multicolumn{14}{|c|}{ Result by method: } & \multirow{2}{*}{\multicolumn{2}{|c|}{ Mutation }} \\
\hline & \multicolumn{4}{|c|}{ Proportion method } & \multicolumn{10}{|c|}{ LiPA } & & \\
\hline & SM & INH & RMP & EMB & TB-P & $\mathrm{S} 1$ & S2 & $\mathrm{S} 3$ & S4 & S5 & R2 & R4a & $\mathrm{R} 4 \mathrm{~b}$ & R5 & LiPA & Seq. \\
\hline 1 & $\mathrm{~S}$ & $\mathrm{R}$ & $\mathrm{R}$ & $\mathrm{R}$ & + & + & - & + & + & + & + & - & - & - & D516V & D516V \\
\hline 2 & $\mathrm{~S}$ & $\mathrm{R}$ & $\mathrm{R}$ & $\mathrm{S}$ & + & + & + & + & + & - & - & - & - & + & S531L & S531L \\
\hline 3 & $\mathrm{~S}$ & $\mathrm{R}$ & $\mathrm{R}$ & $\mathrm{R}$ & + & + & - & + & + & + & + & - & - & - & D516V & D516V \\
\hline 4 & $\mathrm{~S}$ & $\mathrm{R}$ & $\mathrm{R}$ & $\mathrm{S}$ & + & + & + & + & + & - & - & - & - & + & S531L & S531L \\
\hline 5 & $\mathrm{R}$ & $\mathrm{S}$ & $\mathrm{R}$ & $\mathrm{S}$ & + & + & + & + & + & - & - & - & - & + & S531L & S531L \\
\hline 6 & $\mathrm{~S}$ & $\mathrm{~S}$ & $\mathrm{R}$ & $\mathrm{S}$ & + & + & + & - & - & $+1-$ & - & - & - & - & UDR & Deletion 522-525 \\
\hline 7 & $\mathrm{~S}$ & $\mathrm{R}$ & $\mathrm{R}$ & $\mathrm{R}$ & + & + & - & + & + & + & + & - & - & - & D516V & D516V \\
\hline 8 & $\mathrm{~S}$ & $\mathrm{R}$ & $\mathrm{R}$ & $\mathrm{R}$ & + & + & - & + & + & + & + & - & - & - & D516V & D516V \\
\hline 9 & $\mathrm{R}$ & $\mathrm{R}$ & $\mathrm{R}$ & $\mathrm{S}$ & + & + & + & + & - & + & - & - & + & - & H526D & H526D \\
\hline 10 & $\mathrm{~S}$ & $\mathrm{R}$ & $\mathrm{R}$ & $\mathrm{R}$ & + & + & - & + & + & + & + & - & - & - & D516V & D516V \\
\hline 11 & $\mathrm{R}$ & $\mathrm{R}$ & $\mathrm{R}$ & $\mathrm{R}$ & + & + & - & + & + & + & + & - & - & - & D516V & D516V \\
\hline 12 & $\mathrm{R}$ & $\mathrm{R}$ & $\mathrm{R}$ & $\mathrm{R}$ & + & + & - & + & + & + & + & - & - & - & D516V & D516V \\
\hline 13 & $\mathrm{~S}$ & $\mathrm{R}$ & $\mathrm{R}$ & $\mathrm{R}$ & + & + & + & + & + & - & - & - & - & + & S531L & S531L \\
\hline 14 & $\mathrm{R}$ & $\mathrm{R}$ & $\mathrm{R}$ & $\mathrm{R}$ & + & + & + & + & + & - & - & - & - & + & S531L & S531L \\
\hline 15 & $\mathrm{~S}$ & $\mathrm{R}$ & $\mathrm{R}$ & $\mathrm{R}$ & + & + & - & + & + & + & + & - & - & - & D516V & D516V \\
\hline 16 & $\mathrm{~S}$ & $\mathrm{R}$ & $\mathrm{R}$ & $\mathrm{R}$ & + & + & - & + & + & + & + & - & - & - & D516V & S509T/D516V \\
\hline 17 & $\mathrm{~S}$ & $\mathrm{R}$ & $\mathrm{R}$ & $\mathrm{S}$ & + & - & + & + & + & + & - & - & - & - & UDR & Q513K \\
\hline 18 & $\mathrm{~S}$ & $\mathrm{R}$ & $\mathrm{R}$ & $\mathrm{R}$ & + & + & + & + & + & + & - & - & - & - & UD & WT \\
\hline 19 & S & $\mathrm{R}$ & $\mathrm{R}$ & $\mathrm{R}$ & + & + & + & + & + & - & - & - & - & + & S531L & S531L \\
\hline 20 & $\mathrm{R}$ & $\mathrm{R}$ & $\mathrm{R}$ & $\mathrm{S}$ & + & $+/-$ & + & + & + & + & - & - & - & - & UDR & Q513P \\
\hline 21 & $\mathrm{~S}$ & $\mathrm{R}$ & $\mathrm{R}$ & $\mathrm{S}$ & + & + & + & + & + & - & - & - & - & + & S531L & S531L \\
\hline 22 & $\mathrm{~S}$ & $\mathrm{R}$ & $\mathrm{R}$ & $\mathrm{S}$ & + & + & + & + & - & + & - & - & + & - & H526D & H526D \\
\hline 23 & $\mathrm{~S}$ & $\mathrm{R}$ & $\mathrm{R}$ & $\mathrm{R}$ & + & + & - & + & + & + & + & - & - & - & D516V & D516V \\
\hline 24 & $\mathrm{~S}$ & $\mathrm{R}$ & $\mathrm{R}$ & $\mathrm{R}$ & + & + & - & + & + & + & + & - & - & - & D516V & D516V \\
\hline 25 & $\mathrm{~S}$ & $\mathrm{R}$ & $\mathrm{R}$ & $\mathrm{S}$ & + & + & - & + & + & + & + & - & - & - & D516V & D516V \\
\hline 26 & $\mathrm{~S}$ & $\mathrm{~S}$ & $\mathrm{R}$ & $\mathrm{S}$ & + & + & + & + & + & - & - & - & - & + & S531L & S531L \\
\hline 27 & $\mathrm{R}$ & $\mathrm{R}$ & $\mathrm{R}$ & $\mathrm{R}$ & + & + & + & + & + & - & - & - & - & + & S531L & S531L \\
\hline 28 & $\mathrm{R}$ & $\mathrm{R}$ & $\mathrm{R}$ & $\mathrm{R}$ & + & + & + & + & + & + & - & - & - & - & WT & $\mathrm{WT}^{b}$ \\
\hline 29 & $\mathrm{R}$ & $\mathrm{R}$ & $\mathrm{R}$ & $\mathrm{R}$ & + & + & + & + & + & + & - & - & - & - & WT & WT \\
\hline Co. 1 & $\mathrm{~S}$ & $\mathrm{~S}$ & $\mathrm{~S}$ & $\mathrm{~S}$ & + & + & + & + & - & - & - & - & - & - & UDR & R529R \\
\hline Co. 2 & $\mathrm{~S}$ & $\mathrm{~S}$ & $\mathrm{~S}$ & $\mathrm{~S}$ & + & + & + & + & + & + & - & - & - & - & WT & WT \\
\hline Co. 3 & $S$ & S & $\mathrm{S}$ & $\mathrm{S}$ & + & + & + & + & + & + & - & - & - & - & WT & WT \\
\hline Co. 4 & $\mathrm{~S}$ & $\mathrm{~S}$ & $\mathrm{~S}$ & $\mathrm{~S}$ & + & + & + & + & + & + & - & - & - & - & WT & WT \\
\hline Co. 5 & $\mathrm{~S}$ & $\mathrm{~S}$ & $\mathrm{~S}$ & $\mathrm{~S}$ & + & + & + & + & + & + & - & - & - & - & WT & WT \\
\hline Co. 6 & $\mathrm{~S}$ & $\mathrm{~S}$ & $\mathrm{~S}$ & $\mathrm{~S}$ & + & + & + & + & + & + & - & - & - & - & WT & WT \\
\hline H37Rv & $\mathrm{S}$ & $\mathrm{S}$ & $\mathrm{S}$ & $\mathrm{S}$ & + & + & + & + & + & + & - & - & - & - & WT & WT \\
\hline
\end{tabular}

${ }^{a}$ Abbreviations: Co., control; Seq., DNA sequencing of the hypervariable region; S, susceptible; R, resistant; +, positive; -, negative; +/-, indeterminate; UD, undetermined; UDR, undetermined resistant; WT, wild type. Probes R2, R4a, R4b, and R5 are specific for mutations D516V, H526Y, H526D, and S531L, respectively.

${ }^{b} \mathrm{~A}$ V146F mutation was found in this isolate.

ical M. tuberculosis isolates that were pansusceptible for all four first-line antituberculosis drugs were used as controls. All cultures were identified by means of the AccuProbe culture identification test (Gen-Probe Inc., San Diego, Calif.) and conventional biochemical tests $(11,14)$.

Susceptibility testing of all 36 isolates for isoniazid (INH), RMP, ethambutol (EMB), and streptomycin (SM) was carried out by the proportion method on Löwenstein-Jensen medium as described by Canetti et al. (2). The critical concentrations for INH, RMP, EMB, and SM were 0.2, 40, 1.0, and $10 \mu \mathrm{g} / \mathrm{ml}$, respectively. Of the 29 RMP-resistant isolates, only $2(6.9 \%)$ were resistant to RMP alone. Twenty-six $(89.7 \%)$ were also resistant to INH (and thus classified as multidrug resistant), 18 $(62.1 \%)$ were also resistant to EMB, and $9(31.0 \%)$ were also resistant to SM (Table 1). In all, 20 of the 29 (70.0\%) were resistant to at least three of the four first-line drugs.

On the day of detection of growth index 999, 200- $\mu$ l aliquots from Bactec 12B subcultures of the susceptible control and RMP-resistant isolates were incubated at $80^{\circ} \mathrm{C}$ for $1 \mathrm{~h}$ to heat kill the mycobacterial cells. Using primers rpo95 (5'-CCACC CAGGACGTGGAGGCGATCACACCG-3') and rpo397 (5' GTCAACCCGTTCGGGTTCATCGAAACG-3') flanking the 81-bp region of $r p o B(9)$, a 329-bp product was generated from all 36 isolates. The same primers were used for DNA sequencing of both strands using the automated Applied Biosystems 377 DNA sequencer (Applied Biosystems, Foster City, Calif.). A recent study demonstrated that, in some RMP-resistant strains with the wild-type sequence in the 81-bp region, a $\mathrm{V} 146 \mathrm{~F}$ mutation was found in the N-terminal region (7). In order to detect the presence of this mutation, amplification and sequencing were performed using primers Tb176-f $\left(5^{\prime}-\mathrm{C}\right.$ TTCTCCGGGTCGATGTCGTTG-3') and Tb176-r (5'-CGC GCTTGTCGACGTCAAACTC-3') as described previously (7). A 365-bp product was generated and sequenced using the same primers.

The heat-killed samples were also used for production of a biotinylated 256-bp fragment of the $r p o B$ gene using the LiPA kit according to the instructions of the manufacturer (Innoge- 
TABLE 2. Frequency of mutations in RMP-resistant M. tuberculosis isolates from different geographic regions

\begin{tabular}{|c|c|c|c|c|c|c|c|c|c|}
\hline \multirow[b]{2}{*}{ Mutated codon(s) } & \multicolumn{9}{|c|}{$\%$ Mutations in geographic region: } \\
\hline & $\begin{array}{c}\text { Africa }^{a} \\
\text { (reference 22; } \\
n=105 \text { ) }\end{array}$ & $\begin{array}{c}\text { Asia }^{b} \\
\text { (reference 8; } \\
n=77 \text { ) }\end{array}$ & $\begin{array}{c}\text { Australia } \\
\text { (reference } 31 \\
n=33 \text { ) }\end{array}$ & $\begin{array}{c}\text { Brazil }^{c} \\
\text { (reference 27; } \\
n=82 \text { ) }\end{array}$ & $\begin{array}{l}\text { Germany }^{d} \\
\text { (reference 20; } \\
n=36 \text { ) }\end{array}$ & $\begin{array}{c}\text { Greece }^{e} \\
\text { (reference 13; } \\
n=17 \text { ) }\end{array}$ & $\begin{array}{c}\text { Italy } \\
\text { (reference } 18 \\
n=37 \text { ) }\end{array}$ & $\begin{array}{l}\text { United States } \\
\text { (reference } 30 \\
\quad n=61 \text { ) }\end{array}$ & $\begin{array}{l}\text { Hungary }^{f} \\
(n=29)\end{array}$ \\
\hline 572 & & & 3.0 & & & & & & \\
\hline 533 & 3.0 & & & 1.2 & & & & 3.3 & \\
\hline 531 & 53.5 & 46.8 & 48.5 & 53.7 & 36.1 & 47.1 & 56.7 & 34.4 & 31.0 \\
\hline 526 & 21.2 & 18.2 & 30.3 & 20.6 & 22.2 & 17.7 & 29.7 & 39.3 & 6.8 \\
\hline 522 & $10^{g}$ & 1.3 & 6.1 & 2.4 & & & & 1.6 & \\
\hline 521 & & & & & & & & 1.6 & \\
\hline 518 & 1.0 & & & & & & & 1.6 & \\
\hline 516 & 13.1 & 16.9 & 9.1 & 7.4 & 2.8 & 5.9 & 2.7 & 6.6 & 37.9 \\
\hline 514 & & 2.6 & & & & & & 1.6 & \\
\hline 513 & 3.0 & 6.5 & & 1.2 & & & & & 6.8 \\
\hline 511 & 2.0 & 1.3 & & 1.2 & & & & & \\
\hline 508 & & & & & & 17.7 & & & \\
\hline 505 & & & & & & 5.9 & & & \\
\hline 504 & 2.0 & & & & & & & & \\
\hline 531 and 526 & & & & 1.2 & 2.8 & 5.9 & 2.7 & & \\
\hline No mutation & 14.1 & 6.5 & 3.0 & 3.7 & 33.3 & & & 8.2 & $10.3^{h}$ \\
\hline $\begin{array}{l}{ }^{a} \text { South Africa }(n \\
{ }^{b} \text { Bangladesh }(n= \\
(n=21) . \\
{ }^{c} \text { Less common n } \\
517 \text {, and } 518 ; 513 \\
{ }^{d} \text { A rare }(2.8 \%) \\
{ }^{e} \text { Rare }(2.7 \% \text { eac } \\
{ }^{f} \text { A deletion }(522 \\
{ }^{g} \text { Silent mutation } \\
{ }^{h} \text { In one of these }\end{array}$ & $\begin{array}{l}=87) \text {, Sierra } \mathrm{L} \\
=2 \text { ), India }(n=4 \\
\text { utations }(1.2 \% \\
\text { nd deletion } 514 \text {, } \\
\text { louble mutation } \\
\text { h) triple mutatio } \\
-525) \text { that has no }\end{array}$ & $\begin{array}{l}\text { one and Ugand } \\
) \text { Indonesia ( } n= \\
\text { ach) were as fol } \\
515 \text {, and } 516 \text {. } \\
\text { was detected at } \\
\text { ns were detectec } \\
\text { t been reported } \\
\text { N-terminal V14 }\end{array}$ & $\begin{array}{l}\text { a }(n=16) \text {, and } \\
=1), \text { Malaysia }(n \\
\text { lows: } 531 \text { and } 514 \\
\text { codons } 526 \text { and } 5 \\
\text { at codons } 553, \\
\text { previously was d }\end{array}$ & $\begin{array}{l}\text { Namibia }(n=2) \text {. } \\
=14) \text {, Myanmar } \\
4 ; 516 \text { and } 511 ; 51 \\
511 \text {. and } 526 ; 525 \\
\text { letected in one st }\end{array}$ & $\begin{array}{l}n=15), \text { Nepal } \\
5 \text { and } 511 ; 526 \\
\text { 523, and } 516 \text {; } \\
\text { rain }(3.4 \%) \text {. }\end{array}$ & $\begin{array}{l}l=2) \text {, the Phili } \\
\text { nd } 531 ; 524,525 \\
\text { nd } 516,512 \text {, anc }\end{array}$ & $\begin{array}{l}\text { pines }(n=4), \text { Th } \\
\text { and } 526 \text { and dele } \\
511 .\end{array}$ & $\begin{array}{l}\text { ailand }(n=14) \text {, } \\
\text { tion } 527 \text {; deletio }\end{array}$ & and Yemen \\
\hline
\end{tabular}

netics). The biotin-labeled PCR product was denatured and hybridized to a strip with 10 specific oligonucleotide probes (19 to 23 bases long). One probe is specific for the M. tuberculosis complex (TB-P), while five partially overlapping wild-type probes (S1 to $\mathrm{S} 5$ ) encompass the region of the $r p o B$ gene encoding amino acids 509 to 534 . Four other probes are specific for the most common mutations, D516V, H526Y, H526D, and S531L (probes R2, R4a, R4b, and R5, respectively) (Innogenetics). Hybridized PCR product was detected, and the LiPA results were evaluated as described elsewhere (4).

In contrast with previous reports (Table 2) $(8,13,19,20,22$, $26,27,30,31)$, the frequency of occurrence of particular mutations was different in the isolates from East Hungary, with 11 $(37.9 \%)$ isolates carrying the less common D516V mutation. Nine $(31.0 \%)$ isolates had an S531L mutation, and two $(6.9 \%)$ isolates had an H526D mutation. These mutations were also correctly detected in the LiPA. In addition, DNA sequencing identified a double mutation (S509T and D516V) and a deletion (deletion 522-525) that have not been reported in the literature before (32). In these two cases, the LiPA was unable to detect the correct type of the mutation. However, it indicated the presence of the genetic alteration (Table 1). The LiPA also did not reveal the type of mutation in two additional strains with rare mutation patterns (Q513K and Q513P) (Table 1). Moreover, the test provided a false-resistant result with a pansusceptible control strain with a silent mutation (R529R) (Table 1).

The LiPA has been reported to be an easy-to-use test for the rapid detection of RMP resistance. The test is available in a kit format and, therefore, especially useful for routine work in clinical laboratories that are not capable of carrying out DNA sequencing $(4,21)$. In the present study, LiPA was able to detect a genetic alteration in $26(89.7 \%)$ of the 29 RMPresistant strains and to identify the particular mutation in 22 strains $(75.9 \%)$ (Table 1$)$.

The LiPA can provide the type of mutation for only the four most common mutations of the rpoB gene (S531L, H526Y, H526D, and D516V), while in cases of other mutations it indicates only the presence of a genetic alteration. Our findings suggest that in a geographic area such as East Hungary where less common or novel mutations of the $r p o B$ gene occur more frequently, the interpretation of the LiPA results may be more difficult. In such an environment, the characterization of the type of the mutation (and the resultant amino acid change) by DNA sequencing is indispensable in order to avoid the report of any false RMP resistance results.

Although more than $96 \%$ of the RMP-resistant strains have a mutation within the 81-bp core region of the $r p o B$ gene, a recent study revealed that a mutation associated with RMP resistance can also occur in other regions of the gene, although less frequently (7). The present study revealed three RMPresistant isolates where neither the DNA sequencing of the 81-bp region nor the LiPA detected any mutations (Table 1). However, the DNA sequencing for the detection of the V146F mutation was positive in one of these three isolates (strain 28, Table 1). All the other study isolates had the wild-type sequence in this region. The two isolates with no mutations in either region indicate that at present the confirmation of molecular results by conventional tests is still warranted. In addition, since the routinely applied DNA sequencing methods usually examine only the 81-bp region of the $r p o B$ gene (32), in cases where resistance is demonstrated in conventional suscep- 
tibility testing but no mutation is found we also suggest screening for the V146F mutation. If this assay also fails to detect a mutation, then other rare mutations of the $r p o B$ gene, heteroresistance (a mixture of susceptible and resistant strains), or, less likely, another mechanism of resistance may be involved (6, 12, 23).

In conclusion, this study demonstrated that frequencies of particular mutations in RMP-resistant M. tuberculosis isolates from East Hungary are different from those that have been reported for isolates from other geographic areas (Table 2). DNA sequencing of the two regions of the $r p o B$ gene identified mutations in 27 (93.1\%) of the investigated 29 RMP-resistant isolates, and the LiPA identified mutations in 26 isolates $(89.7 \%)$. However, the rapid LiPA was unable to determine the type of mutation in 4 of the 26 strains because these isolates contained unique mutations not included on the test strip. In addition, the one isolate that contained a V146F mutation in the N-terminal region of the $r p o B$ gene was falsely interpreted as RMP susceptible in the LiPA. Finally, the LiPA gave a false-resistant result with one of the RMP-susceptible control strains that contained a silent mutation. These findings demonstrate the importance of validating molecular tests for the detection of RMP resistance using DNA sequence analysis and thus determining the frequencies of particular mutations in the test region before introducing the assay into routine clinical service.

This study was supported in part by grant F-23350 from the Hungarian Scientific Research Fund. Á. Somoskövi was supported by grants 1D43TW00915 and 2D43TW00233 from the Fogarty International Center, National Institutes of Health, Bethesda, Md.

\section{REFERENCES}

1. Annual report of the Hungarian medical care centers in respiratory medicine. 1999. National Korányi Institute for Tuberculosis and Respiratory Medicine, Budapest, Hungary.

2. Canetti, G., W. Fox, A. Khomenko, N. Mahler, N. K. Menon, D. A. Mitchison, N. Rist, and N. A. Smeley. 1969. Advances in techniques of testing mycobacterial drug sensitivity, and the use of sensitivity tests in tuberculosis control programmes. Bull. W. H. O. 41:21-43.

3. Cooksey, R. C., B. P. Holloway, M. C. Oldenburg, S. Listenbee, and C. W. Miller. 2000. Evaluation of the invader assay, a linear signal amplification method, for identification of mutations associated with resistance to rifampin and isoniazid in Mycobacterium tuberculosis. Antimicrob. Agents Chemother. 44:1296-1301.

4. De Beenhouwer, H., Z. Lhiang, G. Jannes, W. Mijs, L. Machtelinckx, R. Rossau, H. Traore, and F. Portaels. 1995. Rapid detection of rifampicin resistance in sputum and biopsy specimens from tuberculosis patients by PCR and line probe assay. Tuber. Lung Dis. 76:425-430.

5. Goble, M., M. D. Iseman, L. A. Madsen, D. Waite, L. Ackerson, and C. R. Horsburgh, Jr. 1993. Treatment of 171 patients with pulmonary tuberculosis resistant to isoniazid and rifampin. N. Engl. J. Med. 328:527-532.

6. Heep, M., B. Brandstatter, U. Rieger, N. Lehn, E. Richter, S. Rusch-Gerdes, and S. Niemann. 2001. Frequency of rpoB mutations inside and outside the cluster I region in rifampin-resistant clinical Mycobacterium tuberculosis isolates. J. Clin. Microbiol. 39:107-110.

7. Heep, M., U. Rieger, D. Beck, and N. Lehn. 2000. Mutations in the beginning of the $r p o B$ gene can induce resistance to rifamycins in both Helicobacter pylori and Mycobacterium tuberculosis. Antimicrob. Agents Chemother. 44: 1075-1077.

8. Hirano, K., C. Abe, and M. Takahashi. 1999. Mutations in the rpoB gene of rifampin-resistant Mycobacterium tuberculosis strains isolated mostly in Asian countries and their rapid detection by line probe assay. J. Clin. Microbiol. 37:2663-2666.

9. Hunt, J. M., G. D. Roberts, L. Stockman, T. A. Felmlee, and D. H. Persing. 1994. Detection of a genetic locus encoding resistance to rifampin in mycobacterial cultures and in clinical specimens. Diagn. Microbiol. Infect. Dis. 18:219-227.
10. Kapur, V., L. L. Li, S. Iordanescu, M. R. Hamrick, A. Wanger, B. N. Kreiswirth, and J. M. Musser. 1994. Characterization by automated DNA sequencing of mutations in the gene $(r p o B)$ encoding the RNA polymerase $\beta$ subunit in rifampin-resistant Mycobacterium tuberculosis strains from New York City and Texas. J. Clin. Microbiol. 32:1095-1098.

11. Kent, P. T., and G. P. Kubica. 1985. Public health mycobacteriology. A guide for a level III laboratory. Centers for Disease Control, Atlanta, Ga.

12. Marttila, H. J., H. Soini, E. Vyshnevskaya, B. I. Vyshnevskiy, T. F. Otten, A. V. Vasilyef, and M. K. Viljanen. 1999. Line probe assay in the rapid detection of rifampin-resistant Mycobacterium tuberculosis directly from clinical specimens. Scand. J. Infect. Dis. 31:269-273.

13. Matsiota-Bernard, P., G. Vrioni, and E. Marinis. 1998. Characterization of rpoB mutations in rifampin-resistant clinical Mycobacterium tuberculosis isolates from Greece. J. Clin. Microbiol. 36:20-23.

14. Metchock, B. G., F. S. Nolte, and R. J. Wallace. 1999. Mycobacterium, p. 399-437. In P. R. Murray, E. J. Baron, M. A. Pfaller, F. C. Tenover, and R. H. Yolken (ed.), Manual of clinical microbiology, 7th ed. ASM Press, Washington, D.C.

15. Mitchison, D. A., and A. J. Nunn. 1986. Influence of initial drug resistance on the response to short-course chemotherapy of pulmonary tuberculosis. Am. Rev. Respir. Dis. 133:423-430.

16. Musser, J. M. 1995. Antimicrobial agent resistance in mycobacteria: molecular genetic insights. Clin. Microbiol. Rev. 8:496-514.

17. Parsons, L. M., J. R. Driscoll, H. W. Taber, and M. Salfinger. 1997. Drug resistance in tuberculosis. Infect. Dis. Clin. N. Am. 11:905-928.

18. Piatek, A. S., S. Tyagi, A. C. Pol, A. Telenti, L. P. Miller, F. R. Kramer, and D. Alland. 1998. Molecular beacon sequence analysis for detecting drug resistance in Mycobacterium tuberculosis. Nat. Biotechnol. 16:359-363.

19. Pozzi, G., M. Meloni, E. Iona, G. Orru, O. F. Thoresen, M. L. Ricci, M. R. Oggioni, L. Fattorini, and G. Orefici. 1999. rpoB mutations in multidrugresistant strains of Mycobacterium tuberculosis isolated in Italy. J. Clin. Microbiol. 37:1197-1199.

20. Rinder, H., P. Dobner, K. Feldmann, M. Rifai, G. Bretzel, S. Rusch-Gerdes, and T. Loscher. 1997. Disequilibria in the distribution of $r p o B$ alleles in rifampicin-resistant $M$. tuberculosis isolates from Germany and Sierra Leone. Microb. Drug Resist. 3:195-197.

21. Rossau, R., H. Traore, H. De Beenhouwer, W. Mijs, G. Jannes, P. De Rijk, and F. Portaels. 1997. Evaluation of the INNO-LiPA Rif. TB assay, a reverse hybridization assay for the simultaneous detection of Mycobacterium tuberculosis complex and its resistance to rifampin. Antimicrob. Agents Chemother. 41:2093-2098.

22. Schilke, K., K. Weyer, G. Bretzel, B. Amthor, J. Brandt, V. Sticht-Groh, P. B. Fourie, and W. H. Haas. 1999. Universal pattern of $r p o B$ gene mutations among multidrug-resistant isolates of Mycobacterium tuberculosis complex from Africa. Int. J. Tuberc. Lung Dis. 3:620-626.

23. Taniguchi, H., H. Aramaki, Y. Nikaido, Y. Mizuguchi, M. Nakamura, T. Koga, and S. Yoshida. 1996. Rifampicin resistance and mutation of the rpoB gene in Mycobacterium tuberculosis. FEMS Microbiol. Lett. 144:103-108.

24. Telenti, A., P. Imboden, F. Marchesi, D. Lowrie, S. Cole, M. J. Colston, L. Matter, K. Schopfer, and T. Bodmer. 1993. Detection of rifampicin-resistance mutations in Mycobacterium tuberculosis. Lancet 341:647-650.

25. Telenti, A., P. Imboden, F. Marchesi, T. Schmidheini, and T. Bodmer. 1993. Direct, automated detection of rifampin-resistant Mycobacterium tuberculosis by polymerase chain reaction and single-strand conformation polymorphism analysis. Antimicrob. Agents Chemother. 37:2054-2058.

26. Traore, H., K. Fissette, I. Bastian, M. Devleeschouwer, and F. Portaels. 2000 Detection of rifampicin resistance in Mycobacterium tuberculosis isolates from diverse countries by a commercial line probe assay as an initial indicator of multidrug resistance. Int. J. Tuberc. Lung Dis. 4:481-484.

27. Valim, A. R., M. L. Rossetti, M. O. Ribeiro, and A. Zaha. 2000. Mutations in the $r p o B$ gene of multidrug-resistant Mycobacterium tuberculosis isolates from Brazil. J. Clin. Microbiol. 38:3119-3122.

28. Victor, T. C., A. M. Jordaan, A. van Rie, G. D. van der Spuy, M. Richardson, P. D. van Helden, and R. Warren. 1999. Detection of mutations in drug resistance genes of Mycobacterium tuberculosis by a dot-blot hybridization strategy. Tuber. Lung Dis. 79:343-348.

29. Watterson, S. A., S. M. Wilson, M. D. Yates, and F. A. Drobniewski. 1998. Comparison of three molecular assays for rapid detection of rifampin resistance in Mycobacterium tuberculosis. J. Clin. Microbiol. 36:1969-1973.

30. Williams, D. L., C. Waguespack, K. Eisenach, J. T. Crawford, F. Portaels, M. Salfinger, C. M. Nolan, C. Abe, V. Sticht-Groh, and T. P. Gillis. 1994. Characterization of rifampin resistance in pathogenic mycobacteria. Antimicrob. Agents Chemother. 38:2380-2386.

31. Yuen, L. K., D. Leslie, and P. J. Coloe. 1999. Bacteriological and molecular analysis of rifampin-resistant Mycobacterium tuberculosis strains isolated in Australia. J. Clin. Microbiol. 37:3844-3850.

32. Zhang, Y., and A. Telenti. 2000. Genetics of drug resistance in Mycobacterium tuberculosis, p. 235-254. In G. F. Hatfull and W. R. Jacobs, Jr. (ed.), Molecular genetics of mycobacteria. ASM Press, Washington, D.C. 
III. 


\title{
Molecular Epidemiology of Drug-Resistant Tuberculosis in Hungary ${ }^{\nabla}$
}

\author{
Csaba Ködmön, ${ }^{1}$ Stefan Niemann, ${ }^{2}$ Judit Lukács, ${ }^{3}$ Éva Sör, ${ }^{3}$ Sándor Dávid, ${ }^{4}$ and Ákos Somoskövi ${ }^{3 *}$ \\ National Public Health and Medical Officers Service, Miskolc, Hungary ${ }^{1}$; Reference Center for Mycobacteria, Forschungszentrum Borstel, \\ Borstel, Germany²; Department of Respiratory Medicine, School of Medicine, Semmelweis University, Budapest, Hungary ${ }^{3}$; and \\ Korányi National Institute for Tuberculosis and Respiratory Medicine, Budapest, Hungary ${ }^{4}$
}

Received 19 June 2006/Returned for modification 23 August 2006/Accepted 30 August 2006

\begin{abstract}
Sixty-eight drug-resistant Mycobacterium tuberculosis isolates (44.2\% of all resistant cases) were analyzed by IS6110 restriction fragment length polymorphism fingerprinting and spoligotyping to provide a deeper insight into the status of drug-resistant tuberculosis in Hungary. A total of 54.4\% of the drug-resistant cases and $75 \%$ of the multidrug-resistant cases could be clustered. Analysis of the spoligotyping patterns of the strains revealed a high rate $(66.2 \%)$ of infection by the Haarlem genotype, while none of the patients were infected by the Beijing genotype. The magnitude and the dynamics of drug-resistant tuberculosis are underestimated in Hungary.
\end{abstract}

One of the greatest concerns of tuberculosis control programs is the emergence and spread of drug-resistant and multidrug-resistant (MDR) (resistance at least to isoniazid [INH] and rifampin [RIF]) tuberculosis. In Hungary in 2003, there were $93(7.9 \%)$ patients with drug-resistant tuberculosis (including MDR tuberculosis) and $16(1.4 \%)$ patients with MDR, and in 2004, the number of drug-resistant and MDR cases was $61(5.1 \%)$ and $9(0.75 \%)$, respectively $(8,9)$. However, it is important that the rate of culture-confirmed cases was only $42.7 \%$ in 2003 and $48.6 \%$ in 2004. Moreover, mandatory susceptibility testing was performed for only $48.8 \%$ of the culturepositive isolates in 2003 and $48.5 \%$ of the culture-positive strains in $2004(8,9)$. Consequently, the actual extent and type of drug-resistant tuberculosis in Hungary are unknown.

Therefore, a retrospective population-based study was performed to provide a molecular insight into the extent of drugresistant tuberculosis in Hungary using DNA fingerprinting analysis. For this purpose, all drug-resistant Mycobacterium tuberculosis complex strains (68 isolates) that were identified in the Hungarian Reference Laboratory for Mycobacteria at the Koranyi National Institute for Tuberculosis and Respiratory Medicine in 2003 and 2004 were analyzed by IS6110 restriction fragment length polymorphism (RFLP) fingerprinting and spoligotyping. These isolates were submitted to the reference center for susceptibility testing from all over Hungary and represented $44.2 \%$ of all drug-resistant tuberculosis cases reported in Hungary in 2003 and $2004(8,9)$.

Testing of all isolates for susceptibility to INH, RIF, ethambutol, and streptomycin (SM) was carried out by the proportion method on Löwenstein-Jensen medium as described previously by Canetti et al. (2). Conventional epidemiologic data were obtained from the database of the National Tuberculosis Surveillance System (NTSC) as described elsewhere previously (13).

\footnotetext{
* Corresponding author. Mailing address: Department of Respiratory Medicine, School of Medicine, Semmelweis University, P.O. Box 250, 1536 Budapest 114, Hungary. Phone: 36-309-633-398. Fax: 36-1214-2498. E-mail: akos@pulm.sote.hu.

${ }^{\nabla}$ Published ahead of print on 13 September 2006.
}

IS6110 RFLP fingerprinting were performed in line with a standardized protocol as described previously $(3,14,17)$. Spoligotyping was performed with a commercially available kit (Isogen Bioscience BV, Maarssen, The Netherlands) according to the instructions of the manufacturer (10). The IS6110 fingerprint and spoligotype patterns of the examined strains were analyzed using Bionumerics software, version 3.5 (Applied Maths, Sint-Martens-Latem, Belgium), as described previously $(3,14,17)$. Clusters were defined as groups of patients with $M$. tuberculosis strains showing identical IS6110 RFLP (same number of IS6110 bands at identical positions [position tolerance, $1.2 \%]$ ) and spoligotype patterns.

The chi-square test and the Mann-Whitney U test were employed to evaluate differences in demographic, epidemiologic, and drug resistance characteristics between clustered and nonclustered patients. Values of $P$ of less than 0.05 were considered significant.

In order to display the degree of relatedness of the isolates, a dendrogram was generated by applying the Dice coefficient and the unweighted-pair group method with arithmetic mean (Fig. 1). Thirty-one isolates (45.6\%) showed unique fingerprint patterns, while 37 isolates (54.4\%) had an IS6110 RFLP pattern and a spoligotyping pattern identical to those of at least one other isolate and were thus grouped into 12 different clusters (Fig. 1). Eleven (16.2\%) patients belonged to the largest cluster (cluster 2), 12 (17.6\%) patients belonged to four smaller clusters with 3 patients each (clusters 1, 4, 10, and 12), and $14(20.6 \%)$ patients belonged to seven clusters with 2 patients each (clusters 3, 5, 6, 7, 8, 9, and 11) (Fig. 1).

Analysis of the spoligotyping patterns revealed that a remarkably high number (45 [66.2\%]) of patients were infected with strains of the Haarlem genotype, while two (2.9\%) patients were infected with an East African-Indian genotype strain, and no classification of major genotypes was possible for $21(30.9 \%)$ isolates (Fig. 1). It is noteworthy that none of the patients were infected by strains of the Beijing genotype.

The characteristics of the 68 human immunodeficiency virus-negative patients with drug-resistant tuberculosis are summarized in Table 1. Statistical analysis did not find any significant difference between clustered and nonclustered patients 
IS6110 fingerprint patterns
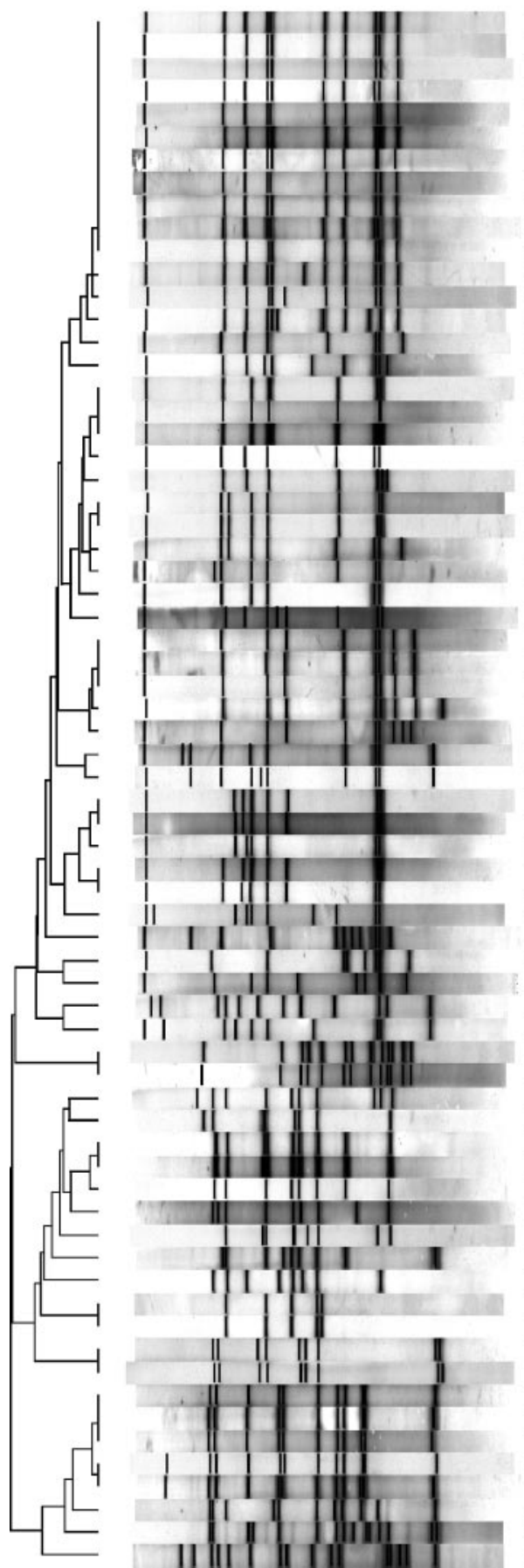

Spoligotype patterns

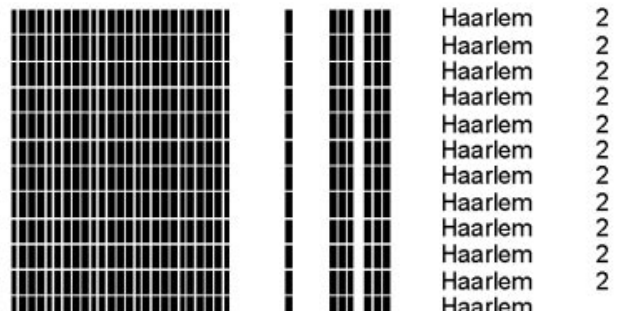

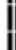

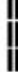

FIG. 1. IS6110 fingerprint and spoligotyping patterns of the 68 patients with drug-resistant tuberculosis. EAI, East African-Indian.

with regard to their demographic characteristics or their medical and social risk factors.

A review of the database of the NTSC revealed that many of the related isolates were from patients located in similar geographic regions. For example, of the 11 patients of cluster 2 (the largest cluster), 5 ( 2 homeless) were from neighboring downtown districts of Budapest (the capital), and 3 were residents of two adjacent, high-incidence, northeast counties
(Heves and Nógrád Counties). Similar coincidences of genotype and geographic location were observed for patients in all other clusters: two of the three patient in cluster 1 , both patients in cluster 3, all three patients in cluster 4, both patients in cluster 5 , both patients in cluster 6 , both patients in cluster 7 , both patients in cluster 8 , two of the three patients in cluster 10 , both patients in cluster 11 , and two of the three patients in cluster 12 were from the same geographic location. Three 
TABLE 1. Characteristics of the 68 clustered and nonclustered patients from Hungary with drug resistant-tuberculosis

\begin{tabular}{|c|c|c|c|c|}
\hline \multirow[b]{2}{*}{ Characteristic } & \multicolumn{3}{|c|}{ No. $(\%)$ of specimens } & \multirow{2}{*}{$\begin{array}{c}P \\
\text { value }^{a}\end{array}$} \\
\hline & $\begin{array}{l}\text { Clustered } \\
(n=37)\end{array}$ & $\begin{array}{l}\text { Nonclustered } \\
\quad(n=31)\end{array}$ & $\begin{array}{c}\text { Total } \\
(n=68)\end{array}$ & \\
\hline Gender & & & & 0.4951 \\
\hline Male & $30(81.1)$ & $23(74.2)$ & $53(77.9)$ & \\
\hline Female & $7(18.9)$ & $8(25.8)$ & $15(22.1)$ & \\
\hline Mean age (yr) & 48.7 & 53.7 & 50.9 & $0.1360^{b}$ \\
\hline Male & 47.5 & 51.9 & 49.4 & \\
\hline Female & 53.9 & 58.9 & 56.5 & \\
\hline Treatment history & & & & 0.9637 \\
\hline No & $30(81.1)$ & $25(80.6)$ & $55(80.9)$ & \\
\hline Yes & $7(18.9)$ & $6(19.4)$ & $13(19.1)$ & \\
\hline Smear microscopy & & & & 0.5148 \\
\hline Positive & $21(56.8)$ & $20(64.5)$ & $41(60.3)$ & \\
\hline Negative & $16(43.2)$ & $11(35.5)$ & $27(39.7)$ & \\
\hline Alcohol abuse & $11(29.7)$ & $9(29.0)$ & $20(29.4)$ & 0.9499 \\
\hline Homeless & $9(24.3)$ & $4(12.9)$ & $13(19.1)$ & 0.2329 \\
\hline Contact & $1(2.7)$ & $1(3.2)$ & $2(2.9)$ & 0.8988 \\
\hline Immigrant & $1(2.7)$ & $1(3.2)$ & $2(2.9)$ & 0.8988 \\
\hline $\begin{array}{l}\text { Resident of congregate } \\
\text { facility }\end{array}$ & $1(2.7)$ & 0 & $1(1.5)$ & 0.3565 \\
\hline
\end{tabular}

patients with MDR tuberculosis were not reported to the NTSC.

Results of susceptibility testing of the 68 resistant isolates by the proportion method are presented in Table 2. Notably, 21 (75.0\%) of the MDR strains were clustered. Comparison of susceptibility patterns of clustered and nonclustered patients revealed a statistically significant difference for mono-SM-resistant $(P=0.0374)$ and $\operatorname{MDR}(P=0.0043)$ patients. In addition, in several clusters, a good correlation was found between the IS6110 fingerprints and the drug susceptibility patterns, implying a close relationship between these strains (data not shown).

The present study is the first to provide a molecular epidemiological insight into the patterns and transmission dynamics of drug-resistant tuberculosis in Hungary. The strains that were included in the study exhibit a medium degree of DNA polymorphism (43 different RFLP patterns in 68 isolates). Since strain diversity is inversely associated with the incidence of the disease, this observation may indicate that the incidence of drug-resistant tuberculosis is actually underestimated by presently available conventional epidemiologic data (7).

The rate of clustered cases $(54.4 \%)$ was much higher than the rate of clustered drug-resistant cases observed in studies performed in Poland $(38.9 \%)$, Belgrade, Central Serbia $(43.5 \%)$, and Germany (33\%), while it was lower than that in Estonia $(67.2 \%)(11,14,15,19)$. The high rate of clustered cases and the high rate $(81.1 \%)$ of new cases among clustered cases indicate that a significant portion of the drug-resistant cases resulted from recent transmission. This result and the
TABLE 2. Drug susceptibility patterns of M. tuberculosis strains isolated from the 68 clustered and nonclustered patients from Hungary with drug-resistant tuberculosis

\begin{tabular}{lllcl}
\hline \multirow{4}{*}{ Drug susceptibility } & \multicolumn{3}{c}{ No. (\%) of specimens } & $P$ \\
\cline { 2 - 4 } & $\begin{array}{c}\text { Clustered } \\
(n=37)\end{array}$ & $\begin{array}{c}\text { Nonclustered } \\
(n=31)\end{array}$ & $\begin{array}{c}\text { Total } \\
(n=68)\end{array}$ & value \\
\hline Mono-INH resistant & $6(16.2)$ & $7(22.6)$ & $13(19.1)$ & 0.5062 \\
Mono-RIF resistant & $1(2.7)$ & $1(3.2)$ & $2(2.9)$ & 0.8988 \\
Mono-SM resistant & $2(5.4)$ & $7(22.6)$ & $9(13.2)$ & 0.0374 \\
Polyresistant (not MDR) & $7(18.9)$ & $9(29.0)$ & $16(23.5)$ & 0.3275 \\
MDR & $21(56.8)$ & $7(22.6)$ & $28(41.2)$ & 0.0043
\end{tabular}

fact that $75 \%$ of the MDR cases were also clustered show that the monitoring and control of drug-resistant tuberculosis is rather inadequate in Hungary (Table 2). The rate of clustered MDR cases was lower in Poland $(50.7 \%)$ and Germany $(49.4 \%)$, was similar in Belgrade $(70 \%)$, and was higher in Estonia $(95.8 \%)(3,11,12,15,19)$.

Although statistical analysis did not reveal any statistically significant association between clustering and the various demographic and epidemiologic characteristics, it is noteworthy that nearly one-third of the clustered patients were alcohol abusers, and almost one-quarter of them were homeless (Table 1). Moreover, since $28.6 \%$ of the MDR cases were homeless and $61 \%$ of the homeless cases were infected by MDR strains, it is clear that, as in other countries (The Netherlands and France), homelessness plays a major role in the transmission of drug-resistant tuberculosis in the capital, where all these cases were found $(6,18)$.

Since contact tracing in Hungary is focused mainly on transmission between close family contacts of diseased individuals, the present study could not determine relationships among all clustered patients. However, the overall correlation of residence and drug resistance profile of patients with clustering strongly supports the close relationship of the strains within a particular cluster.

Another troubling finding of this study was that $3(10.7 \%)$ of the $28 \mathrm{MDR}$ cases were not reported to the NTSC. According to the NTSC, in 2003 and 2004, less than $40 \%$ of the newly diagnosed tuberculosis patients received the mandatory fourdrug regimen from their physicians $(8,9)$. This nonadherence of clinicians to national regulations could also contribute to the increase and transmission of drug-resistant tuberculosis. As the number of patients with drug-resistant tuberculosis in a particular hospital can be low, the lack of familiarity with treatment of patients with drug-resistant tuberculosis might also worsen the situation. Indeed, the importance of this problem was underlined by a recent survey conducted in France. That study revealed a $59 \%$ treatment failure rate for 51 patients with drug-resistant tuberculosis that were treated in a total of 42 different clinical sites, only 35 of which were managed by a respiratory disease specialist (4).

Some M. tuberculosis genotypes, like the W-Beijing and Haarlem families, received special clinical and public health attention because of their greater ability to be transmitted (1, 5). Beijing strains showed a high prevalence in Russia and Estonia ( 30 to $50 \%$ of all cases), while the Haarlem genotype is more prevalent in northern Europe $(20 \%)(11,16)$. Analysis 
of the spoligotyping patterns of the strains revealed that a remarkably high number $(66.2 \%)$ of the patients were infected by strains of the Haarlem genotype, while none of the patients were infected by the Beijing genotype (Fig. 1). It is noteworthy that the presence of the Beijing genotype was observed in every European country from which genotyping results are available (1). These data, and the fact that all but two patients were Hungarian, indicate that in contrast to other European Community countries (i.e., Germany, The Netherlands, and Poland), drug-resistant tuberculosis in Hungary is the result of active in-country circulation of historical clones with European descent (Haarlem family) and not the result of the importation of strains (i.e., Beijing family) from neighboring countries (Romania and former republics of the Soviet Union) with a high rate of Beijing genotype and/or drug-resistant tuberculosis cases.

In conclusion, the results of this study indicate that more effective control steps are needed to detect and intercept the transmission of drug-resistant tuberculosis in Hungary. One solution to the problem could be the introduction of directly observed therapy. The other solution would be the introduction of real-time cohort analysis of cases by the NTSC in order to increase the adherence of clinicians to national guidelines. In addition, the establishment of reference centers, or teams with expert physicians and microbiologists, for consultation and treatment of drug-resistant cases may also be helpful.

C. Ködmön and S. Niemann contributed equally to this study.

Á. Somoskövi was supported by grant D43TW00233 from the Fogarty International Center, National Institutes of Health, Bethesda, MD.

We thank Keith Derbyshire for critical review of the manuscript.

\section{REFERENCES}

1. Brudey, K., J. R. Driscoll, L. Rigouts, W. M. Prodinger, A. Gori, S. A Al-Hajoj, C. Allix, L. Aristimuno, J. Arora, V. Baumanis, L. Binder, P. Cafrune, A. Cataldi, S. Cheong, R. Diel, C. Ellermeier, J. T. Evans, M. Fauville-Dufaux, S. Ferdinand, D. Garcia de Viedma, C. Garzelli, L. Gazzola, H. M. Gomes, M. C. Gutierrez, P. M. Hawkey, P. D. van Helden, G. V. Kadival, B. N. Kreiswirth, K. Kremer, M. Kubin, S. P. Kulkarni, B. Liens, T. Lillebaek, H. M. Ly, C. Martin, C. Martin, I. Mokrousov, O. Narvskaia, Y. F. Ngeow, L. Naumann, S. Niemann, I. Parwati, M. Z. Rahim, V. Rasolofo-Razanamparany, T. Rasolonavalona, M. L. Rossetti, S. RuschGerdes, A. Sajduda, S. Samper, I. Shemyakin, U. B. Singh, A. Somoskovi, R. Skuce, D. van Soolingen, E. M. Streicher, P. N. Suffys, E. Tortoli, T. Tracevska, V. Vincent, T. C. Victor, R. Warren, S. F. Yap, K. Zaman, F. Portaels, N. Rastogi, and C. Sola. 2006. Mycobacterium tuberculosis complex genetic diversity: mining the fourth international spoligotyping database (SpolDB4) for classification, population genetics and epidemiology. BMC Microbiol. 6:23.

2. Canetti, G., W. Fox, A. Khomenko, N. Mahler, N. K. Menon, D. A. Mitchison, N. Rist, and N. A. Smeley. 1969. Advances in techniques of testing mycobacterial drug sensitivity, and the use of sensitivity tests in tuberculosis control programmes. Bull. W. H. O. 41:21-43.

3. Diel, R., S. Schneider, K. Meywald-Walter, C. M. Ruf, S. Rusch-Gerdes, and S. Niemann. 2002. Epidemiology of tuberculosis in Hamburg, Germany: long-term population-based analysis applying classical and molecular epidemiological techniques. J. Clin. Microbiol. 40:532-539.

4. Flament-Saillour, M., J. Robert, V. Jarlier, and J. Grosset. 1999. Outcome of multi-drug-resistant tuberculosis in France: a nationwide case-control study. Am. J. Respir. Crit. Care Med. 160:587-593.

5. Glynn, J. R., J. Whiteley, P. J. Bifani, K. Kremer, and D. van Soolingen. 2002. Worldwide occurrence of Beijing/W strains of Mycobacterium tuberculosis: a systematic review. Emerg. Infect. Dis. 8:843-849.

6. Gutiérrez, M. C., V. Vincent, D. Aubert, J. Bizet, O. Gaillot, L. Lebrun, C. Le Pendeven, M. P. Le Pennec, D. Mathieu, C. Offredo, B. Pangon, and C. Pierre-Audigier. 1998. Molecular fingerprinting of Mycobacterium tuberculosis and risk factors for tuberculosis transmission in Paris, France, and surrounding area. J. Clin. Microbiol. 36:486-492.

7. Hermans, P. W., F. Messadi, H. Guebrexabher, D. van Soolingen, P. E. de Haas, H. Heersma, H. de Neeling, A. Ayoub, F. Portaels, D. Frommel, et al. 1995. Analysis of the population structure of Mycobacterium tuberculosis in Ethiopia, Tunisia, and The Netherlands: usefulness of DNA typing for global tuberculosis epidemiology. J. Infect. Dis. 171:1504-1513.

8. Jónás, J., K. B. Fodor, P. Kiss, M. P. Türgyei, and L. Nyári. 2004. Annual report of the Hungarian medical care centers in respiratory medicine, 2003. Korányi National Institute for Tuberculosis and Respiratory Medicine, Budapest, Hungary.

9. Jónás, J., K. B. Fodor, P. Kiss, M. P. Türgyei, and L. Nyári. 2005. Annual report of the Hungarian medical care centers in respiratory medicine, 2004. Korányi National Institute for Tuberculosis and Respiratory Medicine, Budapest, Hungary.

10. Kremer, K., D. van Soolingen, R. Frothingham, W. H. Haas, P. W. Hermans, C. Martin, P. Palittapongarnpim, B. B. Plikaytis, L. W. Riley, M. A. Yakrus, J. M. Musser, and J. D. van Embden. 1999. Comparison of methods based on different molecular epidemiological markers for typing of Mycobacterium tuberculosis complex strains: interlaboratory study of discriminatory power and reproducibility. J. Clin. Microbiol. 37:2607-2618.

11. Krüüner, A., S. E. Hoffner, H. Sillastu, M. Danilovits, K. Levina, S. B. Svenson, S. Ghebremichael, T. Koivula, and G. Källenius. 2001. Spread of drug-resistant pulmonary tuberculosis in Estonia. J. Clin. Microbiol. 39: 3339-3345.

12. Kubica, T., S. Rusch-Gerdes, and S. Niemann. 2004. The Beijing genotype is emerging among multidrug-resistant Mycobacterium tuberculosis strains from Germany. Int. J. Tuberc. Lung Dis. 8:1107-1113.

13. Mester, J., I. Vadasz, G. Pataki, L. Parsons, T. Fodor, M. Salfinger, and A. Somoskovi. 2002. Analysis of tuberculosis surveillance in Hungary in 2000. Int. J. Tuberc. Lung Dis. 6:966-973.

14. Niemann, S., S. Rusch-Gerdes, and E. Richter. 1997. IS6110 fingerprinting of drug-resistant Mycobacterium tuberculosis strains isolated in Germany during 1995. J. Clin. Microbiol. 35:3015-3020.

15. Sajduda, A., A. Brzostek, M. Poplawska, N. Rastogi, C. Sola, E. AugustynowiczKopec, Z. Zwolska, J. Dziadek, and F. Portaels. 2004. Molecular epidemiology of drug-resistant Mycobacterium tuberculosis strains isolated from patients with pulmonary tuberculosis in Poland: a 1-year study. Int. J. Tuberc. Lung Dis. 8:1448-1457.

16. Toungoussova, O. S., P. Sandven, A. O. Mariandyshev, N. I. Nizovtseva, G. Bjune, and D. A. Caugant. 2002. Spread of drug-resistant Mycobacterium tuberculosis strains of the Beijing genotype in the Archangel Oblast, Russia. J. Clin. Microbiol. 40:1930-1937.

17. van Embden, J. D., M. D. Cave, J. T. Crawford, J. W. Dale, K. D. Eisenach, B. Gicquel, P. Hermans, C. Martin, R. McAdam, T. M. Shinnick, and P. M. Small. 1993. Strain identification of Mycobacterium tuberculosis by DNA fingerprinting: recommendations for a standardized methodology. J. Clin. Microbiol. 31:406-409.

18. van Soolingen, D., M. W. Borgdorff, P. E. de Haas, M. M. Sebek, J. Veen, M. Dessens, K. Kremer, and J. D. van Embden. 1999. Molecular epidemiology of tuberculosis in The Netherlands: a nationwide study from 1993 through 1997. J. Infect. Dis. 180:726-736.

19. Vuković, D., S. Rüsch-Gerdes, B. Savić, and S. Niemann. 2003. Molecular epidemiology of pulmonary tuberculosis in Belgrade, Central Serbia. J. Clin. Microbiol. 41:4372-4377. 
IV. 


\title{
Molecular clues of a microepidemy among homeless tuberculosis patients in Budapest due to a new and local Mycobacterium tuberculosis clade
}

\author{
Csaba Ködmön ${ }^{\mathrm{a}}$, Stefan Niemann ${ }^{\mathrm{b}}$, M. Cristina Gutierrez ${ }^{\mathrm{c}}$, Christophe Sola ${ }^{\mathrm{d}}$, \\ Nalin Rastogi ${ }^{\mathrm{d}}$, Judit Lukács ${ }^{\mathrm{e}}$, Ákos Somoskövi ${ }^{\mathrm{e}, *}$ \\ ${ }^{a}$ National Public Health and Medical Officers Service, Miskolc, Hungary \\ ${ }^{\mathrm{b}}$ National Reference Center for Mycobacteria, Forschungszentrum Borstel, Borstel, Germany \\ ${ }^{\mathrm{c}}$ Laboratoire de Reference des Mycobacteries, Institut Pasteur, Paris, France \\ ${ }^{\mathrm{d}}$ Unité de la Tuberculose et des Mycobactéries, Institut Pasteur de Guadeloupe, Pointe-á-Pitre, Guadeloupe \\ ${ }^{\mathrm{e}}$ Department of Respiratory Medicine, School of Medicine, Semmelweis University, Budapest, Hungary
}

Received 24 April 2007; received in revised form 14 June 2007; accepted 15 June 2007

\begin{abstract}
In Budapest, the capital of Hungary, one of the most important tuberculosis related risk factor is homelessness. The aim of this retrospective study was the genetic characterization of Mycobacterium tuberculosis strains isolated from 66 homeless tuberculosis patients by spoligotyping and mycobacterial interspersed repetitive unit (MIRU) typing. The study identified a local microepidemy in the district with the highest tuberculosis incidence of Budapest. Further genetic characterization has shown that the microepidemy was due to a locally emerged Budapest-specific lineage of $M$. tuberculosis. These data may serve as a reference to better monitor and understand the patterns and transmission dynamics of tuberculosis in this at-risk population and is the first report on genetic diversity of M. tuberculosis in today's Hungarians. The findings also indicate that tuberculosis control and prevention steps among the homeless need to be strengthened.
\end{abstract}

(C) 2007 Elsevier B.V. All rights reserved.

Keywords: Mycobacterium tuberculosis; Spoligotyping; Mycobacterial interspersed repetitive unit; Budapest-specific lineage; Microepidemy; Homeless

\section{Introduction}

The incidence of tuberculosis in Hungary was 24.5 per 100,000 inhabitants (2476 cases) in 2004 (Jónás et al., 2005). Although the number of cases is showing a steady decline since 1996 (42.6 per 100,000 inhabitant [4278 cases]), tuberculosis is continuing to have a major public health impact since the notification rates by county showing significant geographic differences in particular parts of the country (Jónás et al., 2005; Mester et al., 2002). The highest notification rates were observed in three north-eastern counties and in Budapest and the capital region, representing more than 52.5\% (1204 patients) of all reported cases (Jónás et al., 2005). In 2002, we have identified that in Budapest one of the most important tuberculosis related risk factor is homelessness (Lukacs et al., 2004). In that year the incidence of tuberculosis in this at-risk

\footnotetext{
* Corresponding author. Tel.: +36 1355 8682; fax: +36 12142498.

E-mail address: somoskovi@pulm.sote.hu (Á. Somoskövi).
}

population was 676 per 100,000 people which increased to 726 per 100,000 people in 2004 (Jónás et al., 2005; Lukacs et al., 2004; Pataki et al., 2003). In 2004, 53\% of all homeless tuberculosis cases were notified in Budapest, representing $22.3 \%$ of all cases detected in the capital (Jónás et al., 2005). In our previous retrospective study using IS6110 DNA fingerprinting, we also revealed a high rate $(71.2 \%)$ of recent transmission of tuberculosis among the homeless in Budapest and that a considerable portion $(14.9 \%)$ of the patients were referred to hospital from the same shelters (Lukacs et al., 2004). In addition, strains from $16.6 \%$ of the patients (11 individuals) belonged to one major cluster with a unique IS6110 restriction fragment length (RFLP) fingerprint pattern which led to the assumption that this might indicate a microepidemy due to the presence of a local lineage (Lukacs et al., 2004).

The aim of the present study was to confirm this hypothesis by an in-depth analysis of these strains with regard to their origin and relatedness by katG-gyrA gene-polymorphism analysis, spoligotyping and the recently introduced mycobacterial interspersed repetitive unit (MIRU) molecular typing 
method. In addition, an intensive search was performed within an international spoligotyping database (SpolDB4) in order to define the worldwide occurrence and spread of strains of this genotype.

\section{Materials and methods}

Conventional epidemiologic data were obtained from the database of the NTSC as described elsewhere (Mester et al., 2002). Since this retrospective study was using isolates from specimens that were submitted for routine diagnostic purpose, no ethical approval was required.

A total of 66 mycobacterial strains were isolated from 66 newly diagnosed homeless patients with tuberculosis in the Hungarian Reference Laboratory for Mycobacteria at Koranyi Institute for Tuberculosis and Respiratory Medicine in 2002. These cases were representing $47.1 \%$ of all homeless tuberculosis cases in Budapest during the study period (Pataki et al., 2003). All cultures were identified as Mycobacterium tuberculosis complex by means of the AccuProbe culture identification test (Gen-Probe Inc., San Diego, CA) and conventional biochemical tests (Kent and Kubica, 1985; Somoskovi et al., 2000).

Spoligotyping was performed with a commercially available kit (Isogen Bioscience BV, Maarssen, The Netherlands) according to the instructions of the manufacturer (Kremer et al., 1999). Briefly, genomic mycobacterial DNA was directly extracted from cultures by heating at $90{ }^{\circ} \mathrm{C}$ for $30 \mathrm{~min}$. Oligonucleotides DRa and DRb were used as primers to amplify the whole DR region by polymerase chain reaction (PCR). The amplified biotinylated products were hybridized to a set of 43 immobilized oligonucleotides, each corresponding to one of the unique spacer DNA sequences within the DR locus. After hybridization, the membrane was washed, incubated with streptavidin-peroxidase conjugate (Boehringer Mannheim, Indianapolis, Ind.), and finally developed by chemiluminescence. The analysis of spoligotyping data was done in the SpolDB4 Information System which is an automated acces-based labeling and matching system (Garcia de Viedma et al., 2005).
Extraction of mycobacterial DNA for IS6110-RFLP fingerprinting was performed in line with a standardized protocol as described previously (Diel et al., 2002; Niemann et al., 1997; van Embden et al., 1993). The IS6110 fingerprint patterns of the examined strains were analyzed using the Bionumerics software version 3.0 (Applied Maths, SintMartens-Latem, Belgium) as described earlier (Diel et al., 2002; Niemann et al., 1997; van Embden et al., 1993). Clusters were defined as groups of patients with M. tuberculosis strains showing identical RFLP patterns (same number of IS6110 bands at identical positions [position tolerance, 1.3\%]).

The MIRU typing was done as described previously (Supply et al., 2001). MIRU is a PCR-based typing method which assigns the number of tandem repeats in 12 independent loci (MIRUs) that are polymorphic in M. tuberculosis. Therefore, the MIRU type consists of a 12-number code that indicates the number of tandem repeats found for each of the MIRU loci. Clustering analysis between genotypes was performed using Bionumerics version 3.0.

Classification of $M$. tuberculosis isolates into one of the three principal genetic groups delineated by Sreevatsan et al. was performed on the basis of the combination of polymorphisms at codon 463 of the katG gene and at codon 95 of the gyrA gene (Sreevatsan et al., 1997). Polymorphisms were evaluated by a real-time PCR assay with primers and probes designed for use with an Applied Biosystem 7000 real-time PCR system (Applied Biosystems, Foster City, CA) (Rindi et al., 2004).

\section{Results}

The study population consisted of 61 men $(92.4 \%)$ and 5 women $(7.6 \%)$ who were born in Hungary. The patients were from 31 to 73 years old, and the mean age was 47.8 years (men: 48.2 years; women: 44.4 years). All patients were HIV negative.

The spoligotyping patterns of all strains were analyzed by using the SpolDB4 database, a follow-up of the SpoIDB3 project (Brudey et al., 2006; Filliol et al., 2003). Fifty-one (78\%) strains could be classified into 7 shared types [ST50 $(n=17), \operatorname{ST} 1773(n=10)$, ST53 $(n=8)$, ST52 $(n=6)$, ST49 $(n=4), \operatorname{ST62}(n=3), \operatorname{ST} 1771 \quad(n=3)]$ while 15 strains

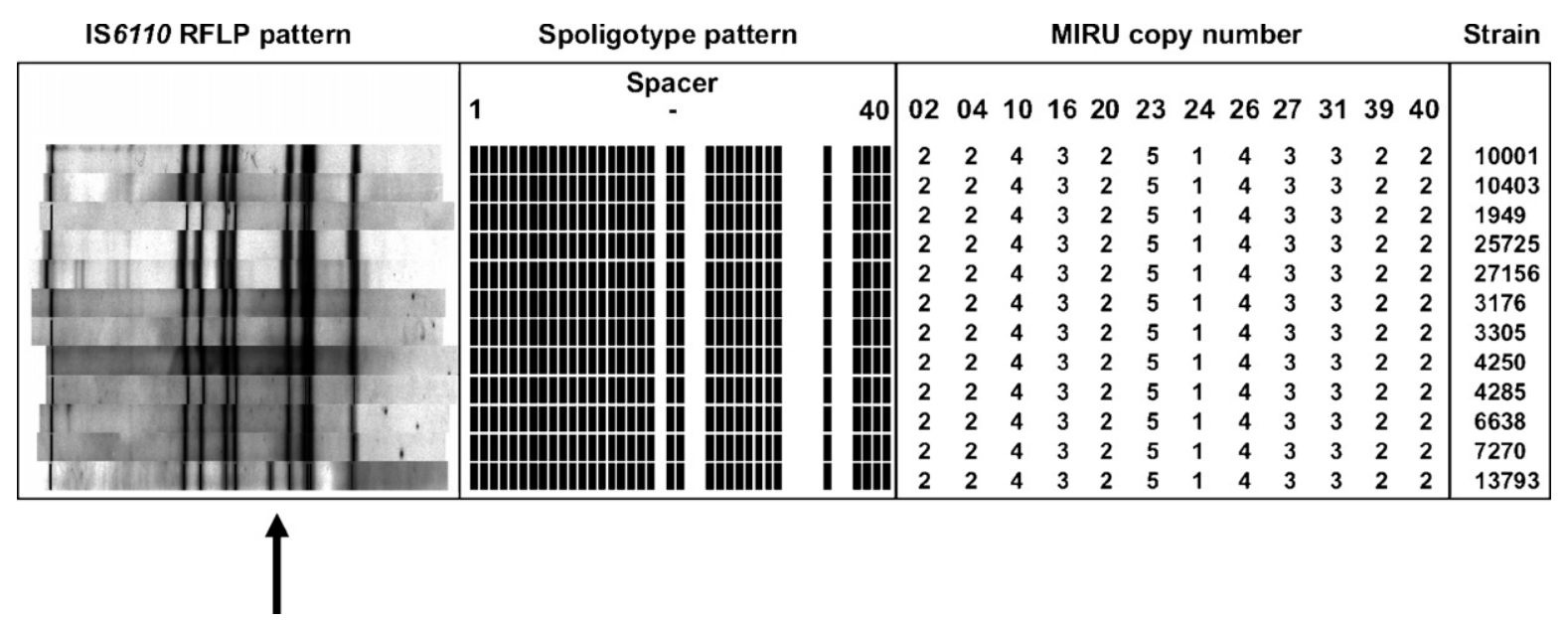

Fig. 1. IS6110 fingerprint, spoligotype and mycobacterial interspred repetitive unit typing patterns of the 12 patients belonging to the Budapest cluster. 
remained orphan (10 pseudo-orphans that were unique in this study but were already found in the database and 5 true orphans). None of the patients were infected by strains of the Beijing genotype. Further analysis of the database revealed that strains belonging to share type ST1773 (777777547760471, octal designation) have a unique spoligotyping pattern (Fig. 1) that may well define a local lineage. The identified pattern is only one spacer different from the pattern of ST370 (7777777477760471) with 18 isolates from Sweden $(n=1)$, Estonia $(n=1)$, USA $(n=2)$, Austria $(n=5)$, Belgium $(n=1)$, Tunisia $(n=1)$, and Brazil $(n=6)$. It is also very close to pattern ST65 (777777777760471) which was first described in Austria and could be a potential ancestor.

By IS6110 RFLP-fingerprint analysis 47 (71.2\%) isolates could be classified into 11 clusters. Thirty-four $(51.5 \%)$ patients belonged to 5 major clusters (size from 4 to 11 individuals), and $13(19.7 \%)$ to 6 smaller clusters with 3 or less individuals. The RFLP-fingerprint analysis of members of shared type ST1773 showed that 11 of the 12 strains belonged to the same IS6110 cluster. Interestingly, there was only 1 band difference between the one IS6110 RFLP non-clustered strain and the 11 strains in the largest cluster (Fig. 1).

In order to characterize more accurately the identified local lineage MIRU typing was performed on these 12 isolates. The MIRU analysis showed identical patterns (pattern 224325143322) for all 12 strains (Fig. 1). When introducing the MIRU findings into the international MIRU provisional web page (http://www.ibl.fr/mirus/mirus.html) the Budapest pattern showed matches only with two other strains of the database. When introduced into the SITVIT database (Institut Pasteur de Guadeloupe, unpublished results), which contains both spoligotyping and MIRU alleles from all-over the world, the Budapest spoligo-MIRU combined pattern did not match with any other strain of the database. However, 20 clinical isolates from the USA and Belgium in the SITVIT database with spoligotyping patterns ST91, ST119, or ST197, all belonging to the X genotype family of strains, shared the same MIT150 pattern of the Budapest clade, reflecting a genetic convergence on or a genetic divergence from this MIRU-type.

Analysis of polymorphisms at codon 463 of the $k a t G$ and at codon 95 of the gyrA in all 12 isolates of share type ST1773 showed that they belong to the Principal Genetic Group 2, which suggests that this clone could be deeply rooted in the $M$. tuberculosis global phylogenetical tree (Filliol et al., 2006; Gutacker et al., 2006).

The retrospective review of the database of the National Tuberculosis Surveillance System (NTSC) revealed that all 12 patients of share type ST1773 were from the VIIIth District of Budapest (one of the poorest districts with the highest tuberculosis incidence). Further investigation of the records found that $2(16.6 \%)$ of the 12 patients were residents of the same homeless shelter.

\section{Discussion}

The incidence of tuberculosis among the homeless shows an alarming increase in Hungary. However, conventional contact tracing of homeless patients is complicated and sometimes even unsuccessful since these individuals are often uncooperative or mobile ( $\mathrm{Li}$ et al., 2003). In a previous retrospective study using IS6110 fingerprinting, we have found a high rate of recent transmission among homeless tuberculosis patients in Budapest similar to other European megapolises (Gutierrez et al., 1998; Lukacs et al., 2004). In the present study, we have extended this investigation and revealed the likelihood of a local microepidemy in a district of Budapest with the highest tuberculosis incidence. Traditional epidemiologic methods showed that $16.6 \%$ of these clustered patients were staying in the same homeless shelter at the time of diagnosing the disease. Although, our survey could not confirm any conventional epidemiologic links between the other cases in the cluster it is very likely that these patients have shared the same day time warming places or other non-overnight out-reach units since most of these homeless facilities are in the VIIIth district of the capital where the patients had lived.

Without the knowledge of genetic diversity of $M$. tuberculosis in other parts of the country and the prevalence of this strain in non-homeless patients, the historical presence of this clade cannot be completely ruled out since complex ecological, social causes and demographical history could also be responsible for the prevalence of some endemic clades throughout long period of times (Day et al., 2001; McGrath, 1988). However, thorough molecular genetic characterization has shown that the observed microepidemy was due to a specific clade that for the time being has not been described elsewhere (Brudey et al., 2006; Filliol et al., 2003). The observed spoligotyping patterns also did not exhibit any similarity to spoligotyping patterns of M. tuberculosis strains that were recently recovered from 18th century Hungarian mummies in a neighboring city of Budapest (Fletcher et al., 2003). Although, additional molecular epidemiologic analysis of strains isolated from non-homeless patients in Budapest and in Hungary will also be necessary to determine the actual prevalence of this genotype, since all the patients were newly diagnosed and none of them were elderly, these cases may well represent newly acquired infections due to an actively spreading clade rather than infections or reactivations of a prevalent local lineage. In addition, the high specificity of the ST1773 clade for Budapest is also evident from its distribution within more than 40,000 isolates of the SpolDB4 database from over 130 countries (Brudey et al., 2006; Filliol et al., 2003; Garcia de Viedma et al., 2005). MIRU typing, presently among the most accurate complementary techniques to demonstrate the clonality of strains, also confirmed the congruence of all 12 isolates.

In conclusion, the present study defined the specific molecular features of a potentially locally emerged $M$. tuberculosis lineage in Budapest that led to a microepidemy among the homeless in the capital. These data may serve as a reference to better monitor and understand the patterns and transmission dynamics of tuberculosis in this at-risk population and more generally is the first report on genetic diversity of $M$. tuberculosis in today's Hungarians. The findings also indicate 
that tuberculosis control and prevention steps among the homeless need to be strengthened.

\section{Acknowledgements}

We thank Thierry Zozio and Marie Gonçalvez for their excellent technical contribution to katG-gyrA typing and spoligotyping, respectively.

\section{References}

Brudey, K., Driscoll, J.R., Rigouts, L., Prodinger, W.M., Gori, A., Al-Hajoj, S.A., Allix, C., Aristimuno, L., Arora, J., Baumanis, V., Binder, L., Cafrune, P., Cataldi, A., Cheong, S., Diel, R., Ellermeier, C., Evans, J.T., FauvilleDufaux, M., Ferdinand, S., Garcia de Viedma, D., Garzelli, C., Gazzola, L., Gomes, H.M., Guttierez, M.C., Hawkey, P.M., van Helden, P.D., Kadival, G.V., Kreiswirth, B.N., Kremer, K., Kubin, M., Kulkarni, S.P., Liens, B., Lillebaek, T., Ho, M.L., Martin, C., Martin, C., Mokrousov, I., Narvskaia, O., Ngeow, Y.F., Naumann, L., Niemann, S., Parwati, I., Rahim, Z., Rasolofo-Razanamparany, V., Rasolonavalona, T., Rossetti, M.L., RuschGerdes, S., Sajduda, A., Samper, S., Shemyakin, I.G., Singh, U.B., Somoskovi, A., Skuce, R.A., van Soolingen, D., Streicher, E.M., Suffys, P.N., Tortoli, E., Tracevska, T., Vincent, V., Victor, T.C., Warren, R.M., Yap, S.F., Zaman, K., Portaels, F., Rastogi, N., Sola, C., 2006. Mycobacterium tuberculosis complex genetic diversity: mining the fourth international spoligotyping database (SpolDB4) for classification, population genetics and epidemiology. BMC Microbiol. 6, 23.

Day, N.P., Moore, C.E., Enright, M.C., Berendt, A.R., Smith, J.M., Murphy, M.F., Peacock, S.J., Spratt, B.G., Feil, E.J., 2001. A link between virulence and ecological abundance in natural populations of Staphylococcus aureus. Science 292, 114-116.

Diel, R., Schneider, S., Meywald-Walter, K., Ruf, C.M., Rusch-Gerdes, S., Niemann, S., 2002. Epidemiology of tuberculosis in Hamburg, Germany: long-term population-based analysis applying classical and molecular epidemiological techniques. J. Clin. Microbiol. 40, 532-539.

Filliol, I., Driscoll, J.R., van Soolingen, D., Kreiswirth, B.N., Kremer, K., Valetudie, G., Dang, D.A., Barlow, R., Banerjee, D., Bifani, P.J., Brudey, K., Cataldi, A., Cooksey, R.C., Cousins, D.V., Dale, J.W., Dellagostin, O.A., Drobniewski, F., Engelmann, G., Ferdinand, S., Gascoyne-Binzi, D., Gordon, M., Gutierrez, M.C., Haas, W.H., Heersma, H., Kassa-Kelembho, E., Ho, M.L., Makristathis, A., Mammina, C., Martin, G., Mostrom, P., Mokrousov, I., Narbonne, V., Narvskaya, O., Nastasi, A., Niobe-Eyangoh, S.N., Pape, J.W., Rasolofo-Razanamparany, V., Ridell, M., Rossetti, M.L., Stauffer, F., Suffys, P.N., Takiff, H., Texier-Maugein, J., Vincent, V., de Waard, J.H., Sola, C., Rastogi, N., 2003. Snapshot of moving and expanding clones of Mycobacterium tuberculosis and their global distribution assessed by spoligotyping in an international study. J. Clin. Microbiol. 41, $1963-$ 1970.

Filliol, I., Motiwala, A.S., Cavatore, M., Qi, W., Hernando Hazbon, M., Bobadilla Del Valle, M., Fyfe, J., Garcia-Garcia, L., Rastogi, N., Sola, C., Zozio, T., Guerrero, M.I., Leon, C.I., Crabtree, J., Angiuoli, S., Eisenach, K.D., Durmaz, R., Joloba, M.L., Rendon, A., Sifuentes-Osornio, J., Ponce de Leon, A., Cave, M.D., Fleischmann, R., Whittam, T.S., Alland, D., 2006. Global phylogeny of Mycobacterium tuberculosis based on single nucleotide polymorphism (SNP) analysis: insights into tuberculosis evolution, phylogenetic accuracy of other DNA fingerprinting systems, and recommendations for a minimal standard SNP set. J. Bacteriol. 188, 759-772.

Fletcher, H.A., Donoghue, H.D., Taylor, G.M., van der Zanden, A.G., Spigelman, M., 2003. Molecular analysis of Mycobacterium tuberculosis DNA from a family of 18th century Hungarians. Microbiology 149, 143-151.
Garcia de Viedma, D., Bouza, E., Rastogi, N., Sola, C., 2005. Analysis of Mycobacterium tuberculosis genotypes in Madrid and identification of two new families specific to Spain-related settings. J. Clin. Microbiol. 43, 17971806.

Gutacker, M.M., Mathema, B., Soini, H., Shashkina, E., Kreiswirth, B.N., Graviss, E.A., Musser, J.M., 2006. Single-nucleotide polymorphism-based population genetic analysis of Mycobacterium tuberculosis strains from four geographic sites. J. Infect. Dis. 193, 121-128.

Gutierrez, M.C., Vincent, V., Aubert, D., Bizet, J., Gaillot, O., Lebrun, L., Le Pendeven, C., Le Pennec, M.P., Mathieu, D., Offredo, C., Pangon, B., Pierre-Audigier, C., 1998. Molecular fingerprinting of Mycobacterium tuberculosis and risk factors for tuberculosis transmission in Paris, France, and surrounding area. J. Clin. Microbiol. 36, 486-492.

Jónás, J., Barsiné, F.K., Kiss, P., Péterfiné, T.M., Nyári, F L., 2005. Annual report of the Hungarian medical care centers in respiratory medicine, 2004. National Korányi Institute for Tuberculosis and Respiratory Medicine, Budapest.

Kent, P.T., Kubica, G.P., 1985. Public Health Mycobacteriology: a guide for a Level III laboratory. Center for Disease Control, Atlanta, GA.

Kremer, K., van Soolingen, D., Frothingham, R., Haas, W.H., Hermans, P.W., Martin, C., Palittapongarnpim, P., Plikaytis, B.B., Riley, L.W., Yakrus, M.A., Musser, J.M., van Embden, J.D., 1999. Comparison of methods based on different molecular epidemiological markers for typing of Mycobacterium tuberculosis complex strains: interlaboratory study of discriminatory power and reproducibility. J. Clin. Microbiol. 37, 2607-2618.

Li, J., Driver, C.R., Munsiff, S.S., Fujiwara, P.I., 2003. Finding contacts of homeless tuberculosis patients in New York city. Int. J. Tuberc. Lung Dis. 7, S397-S404.

Lukacs, J., Tubak, V., Mester, J., David, S., Bartfai, Z., Kubica, T., Niemann, S., Somoskovi, A., 2004. Conventional and molecular epidemiology of tuberculosis in homeless patients in Budapest, Hungary. J. Clin. Microbiol. 42, 5931-5934.

McGrath, J.W., 1988. Social networks of disease spread in the lower Illinois valley: a simulation approach. Am. J. Phys. Anthropol. 77, 483-496.

Mester, J., Vadasz, I., Pataki, G., Parsons, L., Fodor, T., Salfinger, M., Somoskovi, A., 2002. Analysis of tuberculosis surveillance in Hungary in 2000. Int. J. Tuberc. Lung Dis. 6, 966-973.

Niemann, S., Rusch-Gerdes, S., Richter, E., 1997. IS6110 fingerprinting of drug-resistant Mycobacterium tuberculosis strains isolated in Germany during 1995. J. Clin. Microbiol. 35, 3015-3020.

Pataki, G., Megyesi, Á., Fehér, I., 2003. Annual report of the Hungarian medical care centers in respiratory medicine, 2002. National Korányi Institute for Tuberculosis and Respiratory Medicine, Budapest.

Rindi, L., Lari, N., Bonanni, D., Garzelli, C., 2004. Detection of Mycobacterium tuberculosis genotypic groups by a duplex real-time PCR targeting the kat $G$ and gyrA genes. J. Microbiol. Methods 59, 283-287.

Somoskovi, A., Hotaling, J.E., Fitzgerald, M., Jonas, V., Stasik, D., Parsons, L.M., Salfinger, M., 2000. False-positive results for Mycobacterium celatum with the AccuProbe Mycobacterium tuberculosis complex assay. J. Clin. Microbiol. 38, 2743-2745.

Sreevatsan, S., Pan, X., Stockbauer, K.E., Connell, N.D., Kreiswirth, B.N., Whittam, T.S., Musser, J.M., 1997. Restricted structural gene polymorphism in the Mycobacterium tuberculosis complex indicates evolutionarily recent global dissemination. Proc. Natl. Acad. Sci. U.S.A. 94, 9869-9874.

Supply, P., Lesjean, S., Savine, E., Kremer, K., van Soolingen, D., Locht, C., 2001. Automated high-throughput genotyping for study of global epidemiology of Mycobacterium tuberculosis based on mycobacterial interspersed repetitive units. J. Clin. Microbiol. 39, 3563-3571.

van Embden, J.D., Cave, M.D., Crawford, J.T., Dale, J.W., Eisenach, K.D., Gicquel, B., Hermans, P., Martin, C., McAdam, R., Shinnick, T.M., et al., 1993. Strain identification of Mycobacterium tuberculosis by DNA fingerprinting: recommendations for a standardized methodology. J. Clin. Microbiol. 31, 406-409. 